\title{
Acknowledgement to Reviewers of the International Journal of Molecular Science in 2018
}

\author{
International Journal of Molecular Sciences Editorial Office
}

MDPI, St. Alban-Anlage 66, 4052 Basel, Switzerland

Published: 8 January 2019

Rigorous peer-review is the corner-stone of high-quality academic publishing. The editorial team greatly appreciates the reviewers who contributed their knowledge and expertise to the journal's editorial process over the past 12 months. In 2018, a total of 4095 papers were published in the journal, with a median time to first decision of 15 days and a median time to publication of 36 days. The editors would like to express their sincere gratitude to the following reviewers for their cooperation and dedication in 2018:

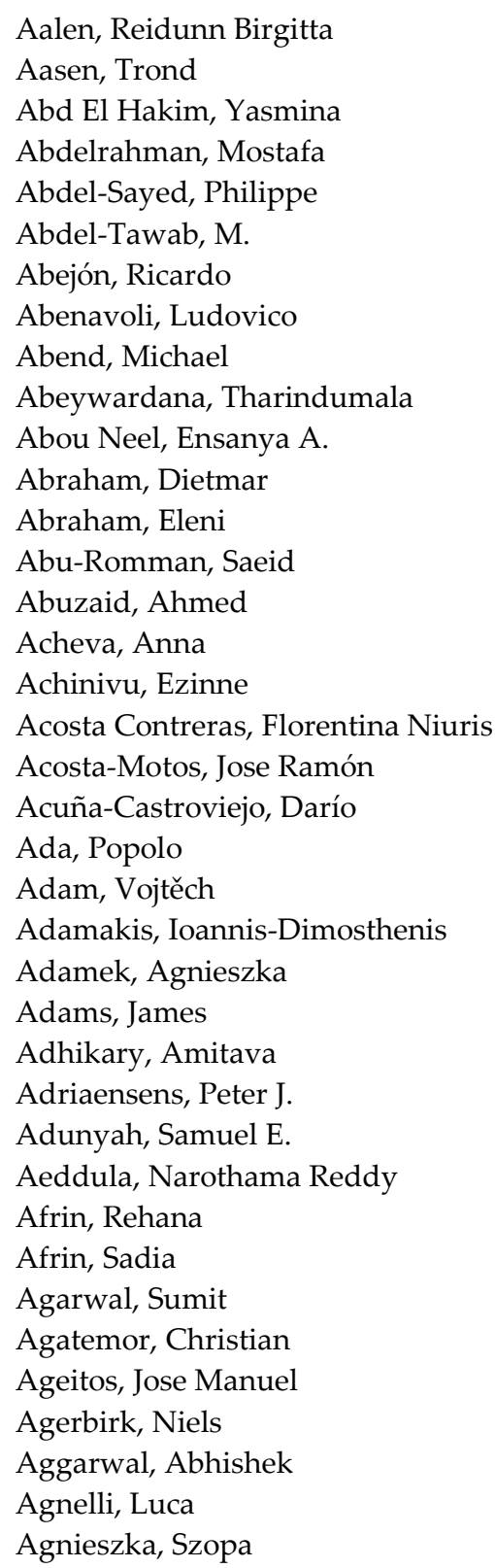

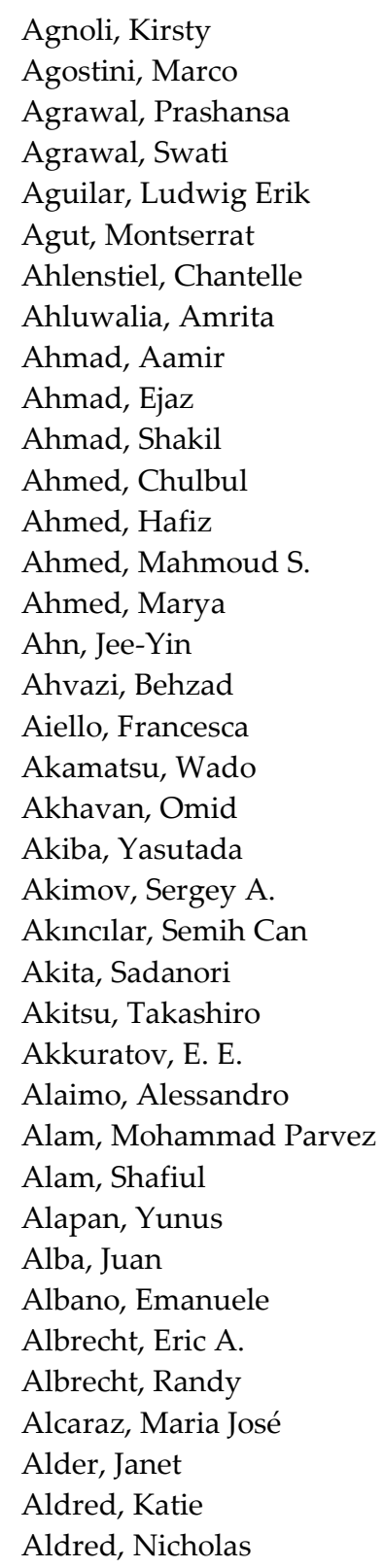


Al-Dujaili, Emad

Aleksandrov, Alexey

Aleman, Ignacio Torres

Alexaki, Vassilia Ismini

Alexander, H. Denis

Alexander, Mathew

Alexander, Rebecca

Alexiou, Christoph

Alfano, Massimo

Alfaro-Moreno, Ernesto

Alfarouk, Khalid

Alferink, Judith

Alfieri, Andrea

Alfieri, Carlo

Alfonsi, Romina

Al-Horani, Rami

Ali, Azahar

Alibakhshi, Mohammad Amin

Alicia, Pickrell

Aliev, Gjumrakch

Allen, Antino

Allen, Caitilyn

Allen, Randy

Allione, Alessandra

Allmang, Christine

Almasan, Alex

Al-Mashhadi, Rozh H.

Almed, Nuzhat

Almeida, Henrique

Almeida, Luciana Oliveira

Almeida, Pedro

Almeida, Rodrigo C.

Almodóvar, Jorge

Almstrup, Kristian

Alonso, Leocadio

Alonso-Magdalena, Paloma

Alpini, Gianfranco

Alpizar, Yeranddy A.

Alqudah, Ahmed

Altomare, Claudia

Alvarez, Carlos

Alvarez, Juan B.

Álvarez-Sánchez, Nuria

Alvarez-Suarez, José

Alves, Eliana

Alves, Sandra

Alzaid, F.

Amadoro, Giuseppina

Amanda, Norvell

Amar, Laurence

Amaral, Alexandra

Amarowicz, Ryszard

Ambati, Jayakrishna

Ambrosio, Maria Raffaella

Ameri, Pietro

Ameye, Maarten

Ami, Diletta

Amillis, Sotiris
Amini-Nik, Saeid

Amirkhani, Masoume

Amital, Howard

Ammendola, Michele

Ammendola, Rosario

Amorós, Asunción

Ampofo, Emmanuel

Amrani, Yassine

Amstislavsky, Sergei

An, Yu

Anandhan, Annadurai

Ananieva, Kalina

Anastasiadou, Maria

Ancona-Contreras, Veronica

Ancuceanu, Robert

Andersen, Catherine

Anderson, Mark T.

Anderson, Sarah N.

Andjelkovic, Anuska V.

Andrade, Arturo

Andrade, Paula B.

Andre, Helder

Andreadou, Ioanna

Andreasson, Erik

Andreev, Konstantin

Andres, Allen M.

Andressen, Kjetil

Andrisani, Ourania M.

Ang, Wee

Angel, Peggi

Angelino, Donato

Angelone, Tommaso

Angeloni, Christina

Angelova, Angelina

Angelova, Mihaela

Angelucci, Adriano

Angermann, Jeff

Aniello, Francesco

Anifandis, George

Anisimova, Irina

Anji, Antje

Anken, Ralf

Anower, Rokebul

Anraku, Makoto

Ansa, Benjamin

Ansaldi, Mireille

Antognelli, Cinzia

Anton, Sonnenberg

Antonangeli, Fabrizio

Antoniadi, Ioanna

Antonio Speretto, Raul

Antonova, Ekaterina Petrovna

Antony, Jane

Anwar, Rokebul

Aoki, Dan

Aoki, Kazuhiro

Aparicio Blanco, Juan

Apone, Fabio 
Appell, Michael

Arai, Ken

Arai, Toshiro

Aran, Gemma

Arana, Ana

Araniciu, Cătălin

Arboleda, Valerie A.

Archacki, Rafal

Ardelean, Lavinia Cosmina

Ardley, Julie

Aref, Amir Reza

Arena, Sabrina

Arevalo, Juan Carlos

Argmann, Carmen A.

Arguelles, Sandro

Argüello, José M.

Arianna, Marucco

Ariga, Katsuhiko

Arimura, Shinichi

Arino, Joaquin

Arispe, Nelson

Ariyoshi, Wataru

Ariza, Maria Eugenia

Armand, Martine

Armani, Andrea

Armanini, Decio

Armentano, Ilaria

Armstrong, Michelle

Arndt, Patrick G.

Arnesano, Fabio

Arnould, Thierry

Arora, Gunjan

Artyszak, Arkadiusz

Aryal, Rishi

Aryal, Uma K.

Asa, Sylvia

Asahi, Michio

Asahina, Kinji

Asai, Akira

Asakura, Tomiko

ASANO, Ryutaro

Asbell, Penny A.

Ascoli Marchetti, Andrea

Ashida, Hitoshi

Askarian-Amiri, Marjan E.

Askes, Sven

Aslam, Muhammad

Aspatwar, Ashok

Aspenström, Pontus

Assirelli, Elisa

Astegno, Alessandra

Atamian, Hagop

Aterini, Stefano

Atkinson, John P.

Atobe, Masakazu

Atsuta, Ikiru

Attanasio, Chiara

Attar, Hooyar
Attarian, Shahram

Atteritano, Marco

Auberger, Patrick

Audet, Céline

Aunapuu, Marina

Aushev, Vasily

Austin, Caroline

Avanesyan, Alina

Avci-Adali, Meltem

Avila, Jesús

Avila-Román, Javier

Avolio, Albert

Aw, Kean

Awayda, Mouhamed

Axpe, Eneko

Aydemir, Tolunay

Ayers, Duncan

Ayyar, Balasubramanium Vijayalakshmi

Azarnia Tehran, Domenico

Azer, Samy A.

Azevedo, Herlander

Azevedo, Nuno F.

Azizi, Ebrahim

Azmi, Asfar S.

Azuma, Mizuki

Azuma, Yoshiaki

Azushima, Kengo

B. Nasrallah, June

Baas, Paul

Baba, Yuh

Babcock, Daniel

Babu, Anish

Babu, R. Jayachandra

Baby, Shine

Bacelar, Eunice

Bach, Duc-Hiep

Back, Kyoungwhan

Bäck, Magnus

Bäck, Nils

Backert, Steffen

Bácskay, Ildikó

Bączek, Tomasz

Baczek-Kwinta, Renata

Badawi, Mohamed

Bado, Igor

Badylak, Stephen F.

Bae, Hae-Rahn

Bae, Hyunsu

Bae, Ki Hyun

Bae, Ok-Nam

Bae, Soochan

Baenas, Nieves

Baer, Patrick C.

Bag, Sudeep

Bagavant, Harini

Baghdadi, Ziad D.

Bagnoli, Paola

Bai, Donglin 


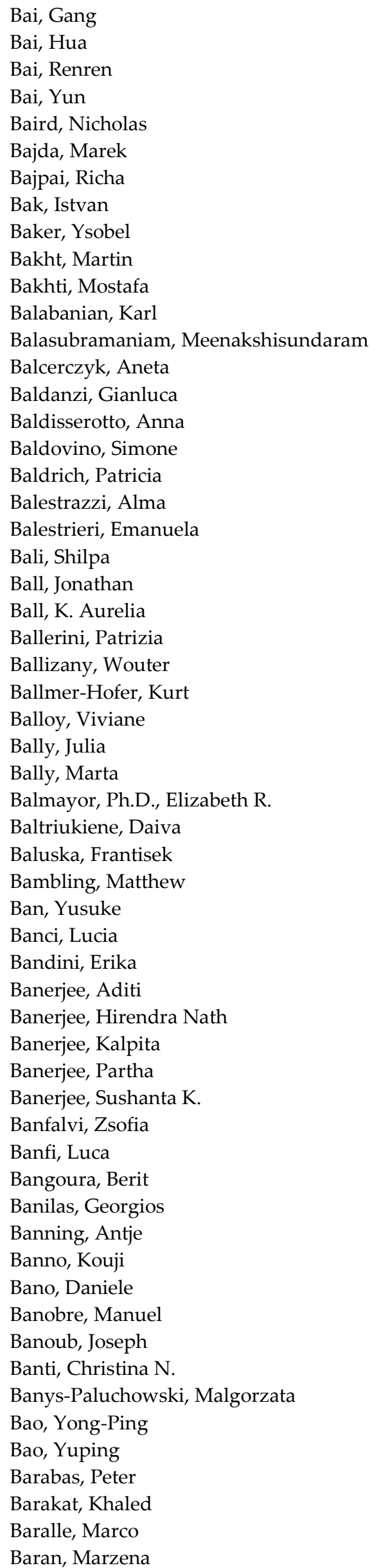

Baranello, Laura

Baranov, Vladislav

Baranowska-Bosiacka, Irena

Bararpour, Taghi

Barata, João

Barault, Ludovic

Barbagallo, Davide

Barbaro, Barbara

Barbeck, Mike

Barber, Paul Richard

Barberi, Tiziano

Barbero, Andrea

Barbier, François F.

Barbieri, Elena

Barbieri, Federica

Barbieri, Silvia S.

Barbillon, Grégory

Barbolina, Maria

Barbosa, Flávia Leão

Barbosa, Judite N.

Barcelo, Juan

Barchetta, Ilaria

Bardet, Michel

Bardi, Giuseppe

Bardy, Cedric

Baregamian, Naira

Barile, Lucio

Barillari, Giovanni

Barker, Gareth R.

Barla, Thirupathi

Barman, Apurba

Barnard, Neal

Barone, Paolo

Barone, Rita

Barral, Duarte

Barrero, Maria J.

Barrio, Rosa

Barros, Francisco

Barros, Pedro M.

Barrott, Jared

Bar-Shavit, Zvi

Barta, Csengele

Bartelt, Alexander

Bartolazzi, Armando

Basak, Debasish

Basak, Sujit

Basco, Leonardo K.

Bascom, Gavin

Bashkin, James K.

Basini, Giuseppina

Basit, Abdul

Baslam, Marouane

Basoli, Valentina

Bassareo, Pier Paolo

Basso, Daniela

Bastarrachea, Luis J.

Basu, Alakananda

Basu, Swaraj 
Batarseh, Yazan

Bates, John T.

Bathula, Chandra

Batsché, Eric

Battaglia, Eric

Battaglia, Fortunato

Battista, Sabrina

Battistini, Lucia

Bauckneht, Matteo

Bauer, Andrew Johann

Baues, Christian

Baulch, Janet

Baumstark-Khan, Christa

Bawadekar, Mandar

Bax, Ben

Bayat, Vafa

Bayer, Philipp

Bayfield, Mark

Bayik, Defne

Bayin, N. Sumru

Bazire, Alexis

Bazylińska, Urszula

Bazzigaluppi, Paolo

Beberashvili, Ilia

Beberok, Artur

Beccano-Kelly, Dayne

Becker, Therese M.

Bécuwe, Philippe

Bednarek, Piotr

Beharry, Kay D.

Behera, Smrutisanjita

Beilby, Mary

Beis, Dimitris

Beitz, Eric

Bekeschus, Sander

Bekri, Soumeya

Belayew, Alexandra

Belenguer, Alvaro

Belfrage, Anna Karin

Belitsky, Jason M

Bell, Stephen

Bellavia, Daniele

Bellen, Hugo J.

Bellosta, Paola

Bellucci, Arianna

Beloor, Jagadish

Bełtowski, Jerzy

Bencsik, Peter

Bendahhou, Saïd

Bendas, Gerd

Benedetti, Michele

Benesic, Andreas

Benhadou, Farida

Benhalima, Katrien

Benini, Stefano

Benistant, Christine

Benjamin, Don

Bennett, James P.
Bentel, Jacqueline M.

Berardi, Anna

Berdis, Anthony J.

Berent-Maoz, Beata

Berenyiova, Andrea

Beretta, Giovanni

Bergamo, Alberta

Bergdahl, Andreas

Berger, Walter

Bergero, Roberta

Bergler, Helmut

Bergonzi, Sara

Berillis, Panagiotis

Berindan-Neagoe, Ioana

Berkowitz, Bruce A.

Berlanga, Ángel Mérida

Berlin, K. Darrell

Bermudez, Marcel

Bernacchioni, Caterina

Bernal, M. Mar

Bernard, Bruno A.

Bernardi, Henri

Bernardo, Letizia

Bernardo, Umberto

Bernitsas, Evanthia

Bernstein, Audrey M.

Berta, Graziella

Bertea, Cinzia

Berthelot, Laureline

Berthod, François

Bertinaria, Massimo

Bertoli, Gloria

Bertrand, Luc

Berzal-Herranz, Alfredo

Bessa, Lucinda

Bessette, Barbara

Besson, Thierry

Betekhtin, Alexander

Betemariam, Senait Ashenafi

Bethke, Gerit

Bettaieb, Ali

Bevan, David

Bevington, Alan

Beyer, Sebastian

Bhalla, Vivek

Bharadwaj, Vivek

Bharti, Kapil

Bhatia, Harsharan S.

Bhatta, Madhav

Bhattacharjee, Sonali

Bhattacharya, Rajat

Bhave, Devayani

Bhavsar, Amit P.

Bhullar, Rajinder P.

Bhusal, Siddhi Jeewan

Biagi, Marco

Biagini, Giuseppe

Biamonti, Giuseppe 
Bianchi, Giuseppe

Bianco, Piero

Biase, Fernando

Biasini, Emiliano

Biasucci, Giacomo

Bichet, Daniel G.

Bidwai, Ashok

Biedermann, David

Biedermann, Thomas

Biernasiuk, Anna

Biersack, Bernhard

Biffis, Andrea

Bigiani, Albertino

Bijak, Michał

Bijlsma, Maarten F.

Billeter, Adrian

Bilodeau, Steve

Birch, John

Birchler, James A.

Bird, Amanda

Birk, Udo

Birman, Serge

Bironaité, Daiva

Bisaga, Maciej

Bishehsari, Faraz

Bisignano, Giuseppe

Bisio, Alessandra

Bišová, Kateřina

Bisti, Silvia

Biswas, Sangita

Biswas, Santanu

Biswas-Fiss, Esther E.

Bitler, Benjamin

Bitonti, Maria B.

Bittel, Douglas C.

Bizzarri, Mariano

Björnsson, Einar

Blacker, Thomas

Blagden, Sarah

Blais, Anne

Blandino, G.

Blank, Volker

Blankesteijn, W. Matthijs

Blanton, Cynthia

Blasi, Juan

Blasiak, Janusz

Błasiak, Janusz

Blein-Nicolas, Mélisande

Block, Anna

Blokland, Arjan

Blomme, Eric

Blomme, Jonas

Blonska, Marzenna

Blume-Jensen, Peter

Blumenberg, Miroslav

Blumenthal, Edward

Boag, Peter

Bobe, Régis
Bocianowski, Jan

Bock, Karl Walter

Bocos, Carlos

Boda, Enrica

Bode, Barrie

Bodenstine, Thomas

Bodily, Jason

Boege, Fritz

Boeglin, Alex

Boekema, Bouke

Boerma, Marjan

Boesze-Battaglia, Kathleen

Bogusławska, Joanna

Bøgwald, Jarl

Boichuk, Sergei

Boitel-Conti, Michèle

Bojarová, Pavla

Bojková, Bianka

Bokareva, Olga S.

Bombard, Sophie

Bommagani, Shbanbabu

Bommakanti, Gayathri

Bonini, Sara Anna

Bonnelye, Edith

Bonnet, Pierre-Antoine

Bonnet, Sébastien

Bonni, Shirin

Bonomini, Francesca

Bonora, Elena

Bontempo, Paola

Boomer, Jonathan S.

Bora-Singhal, Namrata

Borcan, Florin

Borchman, Douglas

Bordin, Luciana

Bordonaba, Jordi Gine

Borer, Katarina T.

Borghaei, Ruth C.

Borghini, Andrea

Borin, Thaiz Ferraz

Borkowski, Leszek

Boros, Laszlo

Borovac, Josip A.

Borsani, Elisa

Bortolotti, Carlo

Bortoluzzi, Stefania

Boscaiu, Monica

Bosch, Maurice

Boschetti, Federica

Boschi-Muller, Sandrine

Boschin, Giovanna

Boscia, Francesca

Bose, Debojit

Bose, Sayantan

Bosland, Maarten C.

Bosnjak, Berislav

Bossard, Pascale

Bosse, Jens B. 
Bossi, Fleur

Bossi, Gianluca

Botelho, Monica C.

Botturi, Andrea

Bouché, Marina

Boudhraa, Zied

Bourgault, Steve

Bousbaa, Hassan

Boutin, Jean

Boutin, Jean A.

Bouvier, Benjamin

Bowen, James

Bowen, Tim

Bowerman, Melissa

Bowling, Heather

Boyer-Guittaut, Michaël

Boyle, Aimee L.

Bozzi, Fabio

Braakhuis, Andrea

Brabec, Viktor

Bracci, Massimo

Bracco, Enrico

Brack, André

Bradbury, Allison

Bradbury, Richard

Brady-Kalnay, Susann M.

Braig, Simone

Brait, Mariana

Branca, Rosa Tamara

Branchini, Alessio

Brandão, Rita Dias

Brandenburg, Klaus

Brandli, Alice

Brands, Michael W.

Brandt, Roland

Bratlie, Kaitlin M.

Braun, Laurence

Bravo, Jeronimo

Brecht, Jeffrey K.

Brehmer, Axel

Breitbart, Haim

Breitenfeld Granadeiro, Luiza

Brem, Jurgen

Bren, Urban

Brender, Jeffrey

Brenner, Annette K.

Brenner, Robert

Brestic, Marian

Brestoff, Jonathan R.

Breuzard, Gilles

Brevern, Alexandre

Brew-Appiah, Rhoda

Brewer, Megan

Brigelius-Flohe, Regina

Brignole, Chiara

Bril, Fernando

Brinchmann, Monica F.

Brink, Peter
Britto, Dev T.

Briza, Peter

Brizzi, Maria Felice

Broderick, Patricia

Broeckling, Corey

Broer, Stefan

Brogi, Simone

Bronisz, Agnieszka

Bross, Peter

Brown, Euan R.

Brown, Geoffrey

Brown, Hannah

Brown, Keith W.

Brown, Robert J.

Brozyna, Anna

Bruce, Alexander W.

Brullo, Chiara

Brumos, Javier

Bruna, Alejandra

Bruneel, Arnaud

Brunelli, Elvira

Brunet, Frédéric

Brunnström, Hans

Bruns, Heiko

Bruschi, Fabrizio

Bruserud, Øystein

Brusslan, Judy

Bryant, Andrew J.

Bryszewska, Maria

Brzóska, Edyta

Brzozowska, Ewa

Brzozowski, Thomas

Bubb, Kristen J.

Bubici, Giovanni

Bubulya, Paula A.

Bucciantini, Monica

Buccigrossi, Vittoria

Buchheim, Judith-Irina

Buchoux, Sébastien

Bucki, Robert

Buckley, M.

Bucolo, Claudio

Budinsky, Robert A.

Budnyak, Tetyana

Budryn, Grażyna

buechler, christa

Bueno, Marta

Buettner, Andreas

Bugla-Płoskońska, Gabriela

Bugliani, Marco

Büki, Andras

Bulli, Peter

Bullon, Pedro

Bunaciu, Rodica P.

Bungartz, Gerd

Bunt, Jens

Buravkova, Ludmila

Burchell, Joy 
Burdo, Tricia H.

Burger, Michael

Burger, Renate

Burghardt, Kyle

Bürglin, Thomas R.

Burgstaller, Joerg

Burke, Dermot

Burke, Richard

Burlingham, William J.

Burns, Jorge S.

Burtea, Carmen

Burtey, Stephane

Burtey, Stéphane

Busby, Chris

Busch, Hauke

Businaro, Rita

Bussolati, Ovidio

Busson, Pierre

Buti, Matteo

Butterfield, Russell J.

Büttner, A.

Bystrowska, Beata

Byun, Jonghoe

Bywater, Robert P.

Caarls, Lotte

Cabrele, Chiara

Cabrera, Adoracion

Cabrera-Fuentes, Hector Alejandro

Caccamo, Daniela

Caccetta, Rima

Cacciotto, Carla

Cacciottolo, Mafalda

Cadamuro, Massimiliano

Caeiro, Maria

Caetano, Nídia

Caffarelli, Carlo

Cahoon, Aubrey Bruce

Cai, Lu

Caiazzo, Elisabetta

Cairrão, Elisa

Caja, Laia

Calarco, Anna

Calcutt, Michael

Caldo, Kristian Mark

Calhelha, Ricardo Costa

Calì, Tito

Calkin, Anna

Call, Mindy

Calle-Pascual, Alfonso L.

Callery, Patrick S.

Calloe, Kirstine

Calvano, Cosima Damiana

Calvo-Lobo, César

Câmara, José Sousa

Camejo, Germán

Camire, Mary

Cammarata, Kirk

Campanini, Barbara
Campbell, Corey

Campbell, Michael

Campbell, Moray

Campione, Marina

Campos, Eric

Campuzano, Oscar

Cancemi, Patrizia

Candido Carvalho, Katia

Cândido, Elizabete

Canela, Núria

Canetta, Elisabetta

Cangkrama, Michael

Canini, Antonella

Cannavo, Alessandro

Cannone, Valentina

Canonico, Marianne

Cansby, Emmelie

Cantalapiedra, Carlos P.

Cantini, Francesca

Cao, Renzhi

Cao, Tongyu

Cao, Weiguo

Cao, Zhe

Capanoglu, Esra

Caparros-Martin, José

Capasso, Raffaele

Capel, Carmen

Capel, Frederic

Capel, Frédéric

Capitanio, Nazzareno

Cappello, Paola

Capranico, Giovanni

Caprini, Marco

Caputo, Gregory A.

Caputo, Roberto

Caraglia, Michele

Carapito, Raphael

Carballo, Iria

Carballo, Sebastian

Carbone, Carmine

Carbonell, Teresa

Carboni, Lucia

Cardenas Zuniga, Roberto

Cardia, Maria Cristina

Cardin, Christine

Cardoso, Fernanda Caldas

Cardoso, Hélia

Cardoso, Susana

Carella, Philip

Caretta, Antonio

Cariati, Federica

Carillo, Petronia

Carlberg, Carsten

Carletti, Paolo

Carlisi, Daniela

Carlsbecker, Annelie

Carmela, Nardelli

Carmosino, Monica 
Carobbio, Stefania

Carol, Pierre

Carosio, Federico

Carotenuto, Marco

Carpaneto, Armando

Carpene, Christian

Carpenter, G. H.

Carpenter, Richard

Carradori, Simone

Carraher, Charles

Carranca, Corina

Carrasco, Pedro

Carregal, Susana

Carrer, Alessandro

Carreras, Joaquim

Carrie, Chris

Carrie, Grueter

Carrillo-Sepulveda, Maria Alicia

Carroll, Patrick

Carroll, Veronica

Carson, Ray

Carta, Fabrizio

Carta, Gianfranca

Cartagena-Rivera, Alexander

Carter, Wayne

Cartland, Sian

Caruso, Calogero

Carvajal Moreno, Fátima

Carvajal, Micaela

Carvalho Pimenta, Daniel

Carvalho, Katherine

Carvalho, Luísa

Casadei, Raffaella

Casal, Margret L.

Casal, Susana

Casals, Núria

Casamassimi, Amelia

Casaretto, Jose A.

Casas, Ana M.

Casati, Lavinia

Cascio, Sandra

Cascione, Mariafrancesca

Caser, Matteo

Casimiro, Sandra

Casini, Giovanni

Cassina, Valeria

Castagna, Michela

Castaldi, Alessandra

Castanera, Raúl

Castanheira, Elisabete M. S.

Castegna, Alessandra

Castellano, Orlando

Castellone, Maria Domenica

Castilho, Alexandra

Castorina, Alessandro

Castresana, Javier

Castro, Antonio Jesus

Castro, Rita
Castrucci, Ana Maria De Lauro

Cattaneo, Fabio

Catucci, Lucia

Cauli, Omar

Cava, Claudia

Cavalcanti, João Henrique F.

Cavallo, Carola

Cavani, Luciano

Cebova, Martina

Ceccarelli, Sara

Ceccherini, Maria Teresa

Cecchetti, Serena

Cecchinato, Valentina

Cécile, Oury

Cecilia, Juan Antonio

Cederbaum, Arthur

Cefalas, A. C.

Cehofski, Lasse

Celiński, Konrad

Celli, Anna

Čènas, Narimantas

Ceolotto, Giulio

Ceranowicz, Piotr

Cerchiara, Teresa

Ceredig, Rhodri

Černý, Jiří

Černý, Martin

Čeřovský, Václav

Cerra, Bruno

Cerutis, Roselyn

Cerutti, Franco

Cervelló, Irene

Cervera, Amelia

César, Ibarra-Alvarado

Cestmir, Altaner

Cha, Chaenyung

Cha, Hee-Jae

Chabé, Magali

Chae, Heeyoung

Chaerle, Laury

Chaganty, Bharat

Chai, Yunrong

Chakrabarti, Kausik

Chakraborty, Arup

Chakraborty, Raja

Chakraborty, Sayan

Chakravorty, David

Chalah, Moussa

Chalah, Moussa Antoine

Chalivendra, Subbaiah C.

Chalkia, Dimitra

Challa, Dilip

Chamcheu, Jean Christopher

Chamonine, Mikhail

Champion, Antony

Chamuleau, Robert A. F. M.

Chan, Ben C. L.

Chan, Chi Ho 
Chan, Chun Wai

Chan, Hon Fai

Chan, Michael WY

Chandaka, Giri Kumar

Chang, Chia-che

Chang, Chien-Chung

Chang, Chuang-Rung

Chang, David

Chang, Fu-Kuei

Chang, Hao-Xun

Chang, Hsueh-Wei

Chang, Hui-hua

Chang, Hung-Yu

Chang, Ing-Feng

Chang, Kee-Lung

Chang, Ko-Tung

Chang, Kuan Y.

Chang, Long-Sen

Chang, Shun-Fu

Chang, Te-Sheng

Chang, Wei-Jen

Chang, Wen-Chi

Chang, Wen-Wei

Chanson, Marc

Chao, Louis Kuoping

Chapman, Eli

Chapoval, Svetlana P.

Charbonneau, Michel

Chardon, Fabien

Charlton, Clivel

Chase, Christine

Chass, Gregory

Chater, Caspar

Chatfield, David

Chatterjee, Nimrat

Chaudhuri, Nazia

Chauhan, Arun

Chaurand, Pierre

Chaves, Elena

Chaves, Raquel

Chaves-Martinez, Felipe Javier

Chavez, Ferman

Che, C. - T.

Checa, Antonio

Chekanova, Julia A.

Chelbi, Sonia T.

Chen, Chiachung

Chen, Chih-Hao

Chen, Chun-Jung

Chen, Chun-Liang

Chen, Guangping

Chen, Guan-Yu

Chen, Guoxun

Chen, Gwo-Shing

Chen, Hongwei

Chen, Hui-Wen

Chen, Jen-Tsung

Chen, Jiangang
Chen, Jianzhong

Chen, Ko Fan

Chen, Kuan-Fu

Chen, Lin

Chen, Ming

Chen, Min-Huey

Chen, Po-chia

Chen, Rong-Jane

Chen, Rui

Chen, Shuyang

Chen, Suzie

Chen, Tianbao

Chen, Tzong-Yueh

Chen, Weiqin

Chen, Wei-Sheng

Chen, Wei-Yu

Chen, Wenchun

Chen, Wenwen

Chen, Xiaozhuo

Chen, Ya-Lei

Chen, Yana

Chen, Yann-Jang

Chen, Yiliang

Chen, Yi-Ning

Chen, Yi-Wen

Chen, Yu-Chih

Chen, Yung-Chih

Chen, Yung-Hsiang

Chen, Yun-Wen

Chen, Zhe

Chen, Zhihang

Cheng, De

Cheng, Haili

Cheng, Hung-Chi

Cheng, Juei-tang

Cheng, Jya-Wei

Cheng, Kuang-Hung

Cheng, Pei

Cheng, Shouyun

Cheng, Xiaoming

Cheng, Xiaoyan

Cheon, Choong-ill

Cheon, Yong-Pil

Cheong, Eun Ju

Cheong, Mi Sun

Cheong, Yong Hwa

Cheong, Young Hun

Chereddy, Kiran Kumar

Cherry, Jonathan

Cheung, Martin

Chhabra, Gagan

Chi, Gerald

Chiabrando, Gustavo Alberto

Chiam, Karen

Chiang, Hsiu-Mei

Chiang, Yi-Ting

Chiao, Ying Ann

Chiappinelli, Katherine 
Chiaraluce, Roberta

Chiaravalli, Anna Maria

Chiarelli, Laurent

Chichger, Havovi

Chiefari, Eusebio

Chiellini, Grazia

Chien, Hung-Yu

Chien, Yin-Hsiu

Chien, Yi-wen

Chiessi, Ester

Chifiriuc, Mariana Carmen

Chilian, William

Chin, Young-Won

Chinnappan, Mahendran

Chio, Christine

Chiocca, Susanna

Chiodini, Iacopo

Chiou, Chun-Tang

Chiricozzi, Andrea

Chitraju, Chandramohan

Chiu, Chun

Chiu, Ing-Ming

Chiu, Joanna

Chiu, Sheng-Chun

Chiu, Wen-Tai

Chiu, Ya-Hui

Chiurchiù, Valerio

Chladek, Grzegorz

Chlichlia, Katerina

Chmurzynska, Agata

Cho, Hongsik

Cho, Hyosun

Cho, Hyunah

Cho, Je-Yoel

Cho, Yoon Hee

Chobot, Vladimir

Choi, Bogyu

Choi, Kyung-Chul

Cholkar, Kishore

Chong, Parkson

Chorley, Brian

Chou, Cheng-Yang

Chou, Feng-Cheng

Chou, Lan-Szu

Choudhary, Kumari Sonal

Choudhury, Malay

Choudry, Haroon A.

Chowdhury, Ananda

Choy, Francis Y. M.

Christ, Bruno

Christ, Torsten

Christensen, Shawn

Christians, Uwe

Christodoulou, Maria-Ioanna

Christoffers, Michael

Chruszcz, Maksymilian

Chu, Ching-Liang

Chu, Jiaxiang
Chu, Pei-Ming

Chu, Shi-Jye

$\mathrm{Chu}$, Xiangping

Chua, Melvin L. K.

Chuang, Hung-Yi

Chui, Kwok Tai

Chun, Kyung-Soo

Chung, Brian K.

Chung, Eun Joo

Chung, Jay $\mathrm{H}$.

Chung, Yong-An

Chwalibog, André

Chwastek, Grzegorz

Chyau, Charng-Cherng

Ciaccio, Marcello

Ciancetta, Antonella

Cianci, Antonio

Ciarimboli, Giuliano

Cicciù, Marco

Ciccone, Marco Matteo

Cicero, Arrigo Francesco Giuseppe

Cichero, Elena

Cichorek, Miroslawa

Ciciliot, Stefano

Cieniewski-Bernard, Caroline

Cieplik, Fabian

Ciereszko, Iwona

Cifone, Maria Grazia

Cigana, Cristina

Cignarella, Andrea

Cignetti, Alessandro

Cimmino, Flora

Cimmino, Giovanni

Cîmpean, Anişoara

Cioffi, Marcelo

Cione, Erika

Ciregia, Federica

Ciriaco, Fulvio

Ciribilli, Yari

Cirilli, Marco

Ciriza, Jesus

Cisowsky, Jaroslaw

Citek, Jindrich

Citro, Valentina

Cividini, Federico

Cizkova, Dasa

Claesson-Welsh, Lena

Clanchy, Felix

Clapp, Lucie H.

Clarke, Anthony

Clarke, Gerard

Clausen, Johannes

Clavé, Pere

Clayton, Andrew

Clelland, Catherine

Clement, Sophie

Climent, Montserrat

Coan, P. M. 
Cobellis, Gilda

Cochis, Andrea

Cognasse, Fabrice

Cohen, Ari J.

Cohen, David

Cohen, Michael

Cohen-Salmon, Martine

Coiffard, Laurence J. M.

Coldea, Teodora

Colecchia, David

Colella, Matilde

Coley, Helen M.

Collavin, Licio

Collier, Jason

Collin, Matthew

Collins, Colm

Collins, Jennifer J. P.

Colombo, Emanuela

Colombo, Monica

Colonna, Giovanni

Colotti, Gianni

Colquhoun, Thomas A.

Coluccia, Mauro

Colussi, GianLuca

Combarnous, Yves

Comi, Cristoforo

Cominelli, Eleonora

Commisso, Mauro

Condorelli, Daniele Filippo

Constantin, Lucian

Conti, Bice

Conti, Heather

Conti, Pio

Contreras, Rúben

Cook, Leah

Cook-Mills, Joan

Cooper, Arthur J. L.

Cooper, Robin

Cooper-Knock, Johnathan

Copolovici, Dana Maria

Coppedè, Fabio

Coquet, Laurent

Corazzari, Marco

Corbet, Cyril

Cordaro, Marika

Cordelier, Sylvain

Corfield, Anthony P.

Corke, Harold

Cormerais, Yann

Cornetti, Luca

Cornillet, Martin

Corrado, Chiara

Corre, Isabelle

Corrigan, Frances

Cortés Castell, Ernesto

Corthay, Alexandre

Corvalan, Alejandro

Cos, Paul
Cosco, Donato

Cosentino, Carlo

Cosme, Marco

Costa, Marina

Costa, Vera Marisa

Costa-Hurtado, Mar

Costantini, Susan

Costantino, Valeria

Cotella, Diego

Cottrell, Jeremy

Coumoul, Xavier

Courtois, Gilles

Coustham, Vincent

Coutinho, João Paulo

Coutinho, Maria Francisca

Covello, Giuseppina

Covino, Roberto

Cowardin, Carrie

Cowell, Ian

Cowell, Rita

Cowin, Allison

Cox, Linda S.

Crawford, Bryan D.

Crawford, Lindsey

Crawford, Lisa

Crawley, Angela M.

Creixell, Teresa Adell

Crescenzi, Marco

Crespan, Emmanuele

Crespo, Mariano Sánchez

Cridge, Andrew

Crisci, Alessandro

Crisponi, Guido

Cristofori-Armstrong, Ben

Crnkovic, Ana

Croce, Anna Cleta

Crompton, Tessa

Crosatti, Cristina

Croset, Martine

Crucho, Carina Isabel Correia

Cruz-Topete, Diana

Csányi, Gábor

Csiszar, Agnes

Csiszár, Agnes

Csősz, Éva

Cubero, Javier

Cucchiarini, Magali

Cuello, Friederike

Cui, Fangda

Cui, Haitao

Cukrov, Dubravka

Cukrowska, Božena

Culi, Joaquim

Cullis, Christopher

Cupellini, Lorenzo

Cuppoletti, John

Curtale, Graziella

Cusanelli, Emilio 
Cutuli, Debora

Cvikl, Doz. Barbara

Cyganowski, Piotr

Cynis, Holger

Czaja, Krzysztof

Czarnecka, Beata

Czeglédi, Levente

Czerwinska, Monika

Czikora, Istvan

Czubryt, Michael P.

Czyż, Jarosław

Czyż, Katarzyna

D'Amico, Agata Graziana

D'Angelo, Paola

Da Cruz, Sandrine

Daberkow, Daberkow

Dacheux, Jean-Louis

Dachineni, Rakesh

D'Acunto, Cosimo Walter

Daczewska, Małgorzata

Dada, Nsa

Dagda, Ruben K.

D'Agostino, Nunzio

Dai, Shaojun

Dai, Yang D.

Daimiel, Lidia

Dakhlallah, Duaa

Dal Ben, Matteo

Dal Col, Jessica

Dal Piaz, Fabrizio

Dalbo, Vincent

Dalin, Martin G.

Dallacosta, Lorenza

Dallinger, Reinhard

Damia, Giovanna

D'Amora, Ugo

Dandekar, Thomas

Dane, Fenny

Daniels, Matthew

Danila, Daniel C.

Danilenko, Michael

Danilov, Alexander

Dansette, Patrick M.

Dante, Silvia

Daras, Gerasimos

Darbani, Behrooz

D'Argenio, Valeria

Darias, María

Darkó, Éva

Darling, April L.

Darling-Reed, Selina F.

Darvell, Brian W.

Das, Aditi

Das, Anindita

Das, Lalitendu

Das, Viswanath

Dasgupta, Kasturi

Dasgupta, Santanu
Dash, Biraja

Dash, Ranjeet

Datta, Poulami

Datta, Rupsa

Daugrois, Jean-Heinrich

Dave, Sandeep

Davey, Matthew P.

David Greg, Riley

David, Alexandre

Davidson, Cristin

Davinelli, Sergio

Davis, Adam

Davis, Catherine

Davletov, Bazbek

Davoli, Roberta

Dawood, Mahmoud A. O.

Dawson, Glyn

Day, David

De Almeida, Andreia

De Bellis, Luigi

De Berardis, Domenico

De Biase, Daniela

De Bock, Marijke

De Carlo, Flavia

De Chiara, Letizia

De Craene, Johan-Owen

De Deurwaerdère, Philippe

De Diego, Nuria

De Feo, Vincenzo

De Filippis, Vincenzo

De Francesco, Ernestina

De Franciscis, Pasquale

De Gonzalo-Calvo, David

De Graaf, Barend H. J.

De Guzman Strong, Cristina

De La Chapelle, Albert

De La Fuente, Hortensia

De La Fuente-Nunez, Cesar

De La Grange, Philippe Brunet

De La Herrán, Roberto

De La Luz Garcia-Hernandez, Maria

De La Varga, Herminia

De Lago, Eva

De Lange, Pieter

De Maagd, Ruud

De Martino, Andrea

De Masi, Luigi

De Matteis, Valeria

De Michelis, Maria Ida

De Moerloose, Philippe

De Morais, Rui Manuel Santos Costa

de Paiva, Cintia

De Pinho, Maria Norberta

De Re, Valli

De Rienzo, Assunta

De Rosa, Maria Cristina

De Santi, Mauro

De Santis, Roberto 
De Smaele, Enrico

De Totero, Daniela

De Vecchis, Renato

De Verdal, Hugues

De Visser, Samuel

De Vita, Alessandro

De Vita, Valerio

De Vries, Carlie

De, Kuntal

De, Pradip

Deaglio, Silvia

Dean, Afshan

Dearnaley, John D. W.

Debeljak, Nataša

DeCoursey, Thomas

Deevska, Gergana

DeFea, Kathryn

Degiacomi, Matteo T.

Degirmenci, Volkan

Degola, Francesca

Deissler, Heidrun

Del Gaudio, Costantino

Del Genio, Charo

Del Giudice, Rita

Del Mistro, Annarosa

Del Re, Brunella

Del Río Celestino, Mercedes

Del Rio, Daniele

Dela Pena, Ike

Delaloy, Celine

Delaney, Colm

Delannoy, Philippe

Deleuze, Herve

Delhanty, Patric

Delihas, Nicholas

Delisle, Jean-Sébastien

Dellavia, Claudia

Delle Monache, Simona

Dellis, Olivier

Dello Ioio, Raffaele

Delogu, Giovanna

Delogu, Lucia

Delorme, Vincent

Demarquoy, Jean

Demir, Ihsan Ekin

Demirhan, Erhan

Demyanets, Svitlana

Deng, Win-Ping

Denis, Sereno

Denisov, Ilia

Denko, Nicholas

Deokar, Hemantkumar

Deplazes, Evelyne

Der Schaff, Arjen Van

Deriu, Marco Agostino

DeRosa, Maria

D'errico, Stefano

Deryusheva, Svetlana
Desai, Jigar

Desai, Varsha G.

Desando, Giovanna

Desantis, Vanessa

Deshmukh, Rupesh

Deshmukh, Umesh S.

Deshpande, Shayu

Desmet, Tom

Després, Charles

Dessain, Scott

Desvergne, Béatrice

Dettweiler, Ulrich

Deuss, Peter

Devarajan, Priyadharshini

Devasundaram, Santhi

Devesa, Jesús

Devine, Patrick J.

Devireddy, Amith

Devy, Jérôme

Dey, Bijan K

Dhakal, Dipesh

Dhamija, Sonam

Dhanasekaran, Danny N.

Dhariwal, Raman

Dharker, Nachiket

Dharmawardhane, Suranganie

Dhawan, Deepika

Di Bartolomeo, Antonio

Di Donato, Marzia

Di Fazio, Pietro

Di Franco, Manuela

Di Giulio, Massimo

Di Liegro, Italia

Di Marcotullio, Lucia

Di Napoli, Arianna

Di Palma, Michael

Di Pierro, Prospero

Di Pietro, Cinzia

Di Primo, Carmelo

Di Renzo, Livia

Di Sansebastiano, Gian Pietro

Di Zazzo, Erika

Dias, George J.

Díaz Rodríguez, Isabel

Diaz, Francisca

Diaz-Rodriguez, Patricia

Dibb, Nicholas

Didiášová, Miroslava

Didonna, Alessandro

Die, Jose

Dieckmann, Klaus-Peter

Diederich, Marc

Diekman, Alan

Diermeier, Sarah

Dijkhuizen, Lubbert

Dijkstra, Bauke W.

Dillman, Adler R.

Dilokpimol, Adiphol 
DiMattia, Gabriel

Dimitroff, Charles J.

Dimmock, Jonathan R.

Dimopoulos, Konstantinos

Ding, Baoqing

Ding, Cherlyn

Ding, Jianxun

Ding, Lin

Ding, Tianbing

Ding, Zhaoyang

Dings, Ruud P. M.

Dinh, Dzung $\mathrm{H}$.

Dinischiotu, Anca

Dinulescu, Daniela M.

Dinwiddie, Darrell L.

Diomede, Francesca

Dionisio, Giuseppe

Dioum, El Hadji M.

Dioum, Elhadji M.

DiPaolo, Richard J.

Disdier, Clemence

Distel, Martin

DiTacchio, Luciano

Dittmer, Neal T.

Divi, Rao L.

Dixon, Dan A.

Dixon, David A.

Dixon, Ian M.C.

Dizdaroglu, Miral

Dkhar, Hedwin Kitdorlang

Dluzen, Douglas

Do, Duy Ngoc

Do, Sun Hee

Do, Yi-Yin

Dobrikova, Anelia G.

Dobson, Renwick C. J.

Docimo, Teresa

Dockrell, Hazel

Doddapaneni, Ravi

Doddapattar, Prakash

Dodds, W. Jean

Doekes, Gert

Doi, Kazuya

Dolga, Amalia

Doliana, Roberto

Dolzhenko, Anton

Dombrovsky, Aviv

Domenicotti, Cinzia

Domigan, Laura Joy

Domingo-Calap, Pilar

Domínguez Rebolledo, Álvaro Efrén

Domínguez-Álvarez, Enrique

Domozych, David S.

Donadel, Giulia

Donadelli, Massimo

Donaire, Livia

Dong, Lanfeng

Dong, Shihui
Dong, Xiao

Dong, Yibo

Dongiovanni, Paola

Donkersley, Philip

Donnelly, Deirdre

Donners, Marjo

Donninger, Howard

Donnini, Sandra

Donno, Dario

D'Onofrio, Nunzia

Donzelli, Elisabetta

D'Orazio, John

Dorey, C. Kathleen

Dormond, Olivier

D'Orsi, Beatrice

Dos Santos, José C. S.

Dos Santos, Reinaldo Sousa

Dosoky, Noura

Dosoky, Noura S.

Dostmann, Wolfgang

Doudin, Khalid

Doulgeraki, Agapi

Douros, Jonathan D.

Dovat, Sinisa

Dovinova, Ima

Dovizio, Melania

Downie, J. Allan

Downs, Diana M.

Doyle, Hester

Doyle, Siamsa

Doyle, Todd

Dr. Grant, William

Drago, Francesco

Dragomir, Mihnea

Drancourt, Michel

Drawert, Brian

Drenjančević, Ines

Dresch, Jacqueline

Drevet, Richard

Dreyer, Ingo

Dreyfus, Cheryl

Driscoll, James J.

Driver, John

Drougard, Anne

Drummond, Rebecca

Drysdale, Thomas

Du, Caigan

Du, Deguo

Du, Wa

Du, Xiao-jun

$\mathrm{Du}, \mathrm{Xin}$

Du, Xingrong

Duan, Bin

Duarte, Andreia

Duarte, José M.

Duarte, Maria Cristina

Dubey, Mukesh K.

Dubielecka, Patrycja M. 
Dubois, Annick

Dubrez, Laurence

Duclot, Florian

Duda, Małgorzata

Dudas, Jozsef

Dudnikov, Alexander

Dudok, Jeroen

Duffy, Aaron M.

Dugé De Bernonville, Thomas

Duh, Pin-Der

Duina, Andrea A.

Dumitru, Andra-Cristina

Dun, Matthew D.

Dungan, Cory

Dunner, Susana

Dunphy, Michael J.

Duong, Haiminh

Duranton, Christophe

Durazzo, Alessandra

Durek, Thomas

Duryee, Michael J.

Dusi, Veronica

Dutta, Arijit

Dutta, Arnob

Dutta, Palash

Duwaerts, Caroline

Dvorakova-Hortova, Katerina

Dwinell, Michael B.

Dwivedi, Chandrahar

Dyakin, Victor V.

Dyall, Julie

Dyr, Jan

Dzemidchyk, Vadzim

Dziedzic, Arkadiusz

Dziegiel, Piotr

Dziegielewska, Barbara

Dziewit, Lukasz

Dzimitrowicz, Anna

Dziubla, Thomas

Dzobo, Kevin

Eaton, Douglas

Ebara, Mitsuhiro

Eberini, Ivano

Ebert, Thomas

Ebihara, Lisa

Eble, Johannes

Ecke, Thorsten

Edamakanti, Chandrakanth

Edlich, Frank

Edwards, Martin

Efferth, Thomas

Efird, Jimmy

Efremenko, Elena

Egawa, Gyohei

Egger, Boris

Ehlen, J. Christopher

Ehmer, Ursula

Eichstaedt, Christina
Eid, Nabil

Eiler, Daniel R.

Einkauf, Jeffrey D.

Einspanier, Ralf

Eisel, Ulrich

Ekberg, Jenny

Ekielski, Adam

Ekiert, Halina

Eklund, Erik A.

El Aidy, Sahar

El Bairi, Khalid

El Kasmi, Farid

El Muayed, Malek

El-Bacha, Tatiana

Elbasyoni, Ibrahim

Eldeeb, Mohamed

Eleftheriadis, Theodoros

Eleftheriou, Eleftherios P.

Eleni, Anastasiadou

Eleonora, Leucci

El-Esawi, Mohamed

Elferink, Cornelis

El-Gazzar, Mohamed M.

Elghobashi-Meinhardt, Nadia

Elia, Ilaria

Eliopoulos, Elias E.

El-Kadi, Ayman

Elkon, Ran

Elleuche, Skander

Elli, Stefano

Ellsworth, Buffy

El-Naggar, Adel

El-Seedi, Hesham R.

Elshimali, Yahya I.

Elson, Ari

Empl, Michael

Emri, Eszter

Endo, Makoto

Enemark, Eric

Engel, Andrew

Engelberg, David

Engelberth, Jurgen

Engelsdorf, Timo

Englezos, Vasileios

Enguita, Francisco

Enguita, Francisco J.

Enos, Reilly T.

Enrich, Carlos

Enugutti, Balaji

Eom, Gwang Hyeonx

Eppler, Elisabeth

Epstein, Alan L.

Erb, Randall

Erceg, Slaven

Erdmann, Kati

Erickson, Michelle

Erlandsson, Malin C.

Ernst, Wolfgang 
Erny, Daniel

Errington, Timothy M.

Ertaylan, Gokhan

Escobar, Carolina

Escobar, Matthew A.

Escobedo-Lucea, Carmen

Escola-Gil, Joan Carles

Escudero, Nuria

Eskina, Erika N

Espada, Jesús

Espadas Villanueva, Isabel

Espel, Enric

Espen, Luca

Esposito, Ciro

Esposito, Emilio Xavier

Esposito, Francesco

Espuny, Antoni Camins

Esque, Jérémy

Estavillo, Gonzalo

Esteban, Susana

Estelrich, Joan

Estensoro, Itziar

Esteve-Romero, Josep

Esteves, Ivania

Estevez, Jorge D.

Estévez, Raúl

Estour, François

Etalo, Desalegn

Eubank, Timothy

Eufemi, Margherita

Éva, Csaba

Evans, Gethin

Eves-van Den Akker, Sebastian

Evrard, Solène M.

Ezhov, Marat V.

Ezura, Yoichi

Fabbretti, Attilio

Fabian, Carol

Fabiani, Roberto

Fabio Zuluaga, Hector

Fabris, Linda

Fabris, Luca

Fabrizius, Andrej

Facchiano, Angelo

Fagerstedt, Kurt

Faggio, Caterina

Fahraeus, Robin

Failla, Cristina M.

Fairweather, Stephen

Faísca, Patrícia

Faiyue, Bualuang

Fakhoury, Ahmad

Falahati Anbaran, Mohsen

Falcieri, Elisabetta

Falcone, Germana

Falconer, Robert

Fales, Andrew

Falkinham, Joseph
Fan, Chenguang

Fang, Junnan

Fang, Mingxi

Fang, Xiaolan

Fang, Yi

Fanizzi, Francesco Paolo

Fantini, Jacques

Fantini, Sebastian

Fantuzzi, Laura

Farah, shady

Fare, Silvia

Fareleira, Paula

Faria Albanese, Jimmy Alexander

Farnoud, Amir

Farolfi, Alberto

Farr, Christine J.

Farrar, Kerrie

Farrar, Mark

Farrugia, Brooke

Fasinu, Pius S.

Fattuoni, Claudia

Fauque, Patricia

Fauré, Julien

Fazakerley, Daniel

Febbraio, Ferdinando

Fedele, Francesco

Federico, Antonio

Fedorova, Olga V.

Fehrenbacher, Jill C.

Feichtinger, Georg

Felgueiras, Helena

Felix, Klaus M.

Felley-Bosco, Emanuela

Fend, Falko

Feng, Chuang

Feng, Jing

Feng, Zhi Chao

Feo, Francesco

Feraco, Alessandra

Ferchaud, Anne-Laure

Ferens-Sieczkowska, Mirosława

Feresin, Rafaela G.

Ferguson, Bradley

Ferguson-Miller, Shelagh

Fernandes, Maria Helena

Fernandez, Agustin F.

Fernández, Ignacio

Fernández, Irene García

Fernández, José A.

Fernandez-Alfonso, Maria

Fernández-Ballester, Gregorio

Fernandez-Botran, Rafael

Fernandez-Jimenez, Nora

Fernández-Lafuente, Roberto

Fernández-López, Manuel

Fernandez-Martinez, Lorena

Fernández-Mateos, Pilar

Fernández-Ponce, M. Teresa 
Fernández-Ruiz, Javier

Fernandez-Valle, Cristina

Fernando, Danilo

Ferrage, Fabien

Ferrante, Antonio

Ferrante, Claudio

Ferrante, Patrizia

Ferrari, Paola

Ferrario, Valerio

Ferraz, Ricardo

Ferreira, Paula M.

Ferreon, Allan Chris M.

Ferreri, Carla

Ferretti, Elisabetta

Ferri, Giovanni Maria

Ferrigno, Andrea

Ferris, William

Ferrus, Alberto

Festuccia, Nicola

Figee, Martijn

Figueiredo, Andreia

Figueiredo, Marxa L.

Figueroa-Romero, Claudia

Filaire, Edith

Filannino, Pasquale

Filek, Maria

Filella, Xavier

Filgueira, Luis

Filhol, Odile

Filigheddu, Nicoletta

Filosto, Massimiliano

Fine, James Burke

Finelli, Carmine

Finkemeier, Iris

Finšgar, Matjaž

Finsterer, Josef

Fiocchetti, Marco

Fioravanti, Antonella

Fiori Gradia, Daniela

Fiorito, Silvana

Fiorotto, Romina

Firestein, Bonnie

Fischer, André

Fischer, Nicole

Fischer-Fodor, Eva

Fisher, Scott

Fisslthaler, Beate

Fiszer, Agnieszka

Fiume, Giuseppe

Fixen, Kathryn

Flack, John

Flavell, David J.

Fletcher, Nicola F.

Florczyk, Stephen J.

Flores, Francisco B.

Floresta, Giuseppe

Flórez, Laura V.

Florin, Luise
Florio, Tullio

Flournoy, Nikaela

Fogarty, Gerald

Fojt, Lukas

Fojtová, Miloslava

Folco, Hernan Diego

Folini, Marco

Fölster-Holst, Regina

Fonseca, Ana

Fonseca, Bruno

Fonseca, Carla

Fonseca, Filomena

Fontana, Luca

Fontana, Robert J.

Font-Burgada, Joan

Fontes, Paulo

Foo, Eloise

Forcales, Sonia-V.

Forget, Aurelien

Forghieri, Fabio

Formanek, Pavel

Formisano, Luigi

Fornara, Fabio

Forn-Cuni, Gabriel

Forneris, Federico

Forrest, Marc

Forster, Britta

Forsyth, Christopher B.

Fort, Patrice E.

Forte, Eleonora

Forte, Elvira

Fortea-Verdejo, Marta

Foster, Tim

Foster, Warren G.

Fosu-Nyarko, John

Fouassier, Laura

Fountain, Samuel J.

Fournier, Neil

Fourtounas, Costas

Fousteris, Manolis

Fox, Elizabeth

Fox, Glen

Frago, Enric

Frame, Leigh A.

Francés-Soriano, Laura

Francisco, Javier López-Baena

Franco, Aime T.

Franco, Albina Cristina Ribeiro

Franco, Diego

Franco, Luis

Franco, Omar

Franco, Rodrigo

Francolini, Maura

Francuz, Tomasz

Frank, Saša

Franken, Nicolaas A. P.

Frankenberg, Stephen

Franko, Andras 
Fraser, Christopher S.

Frazzi, Raffaele

Frederix, P. W. J. M. (Pim)

Freedman, Holly

Freeman, Linnea R.

Freeze, Hudson $\mathrm{H}$.

Freire, Joao

Frese, Steven A.

Frieboes, Hermann

Friml, Jiri

Frkanec, Ruža

Froeyen, Matheus

Froeyen, Mathy

Fröhlich, Eleonore

Frolov, Andrej

Fromen, Cathy

Frossard, Jean-Pierre

Frugis, Giovanna

Frungillo, Lucas

$\mathrm{Fu}$, Shih-Feng

$\mathrm{Fu}$, Shulan

$\mathrm{Fu}$, Yangxin

$\mathrm{Fu}, \mathrm{Yu}$

Fuchs, Marc

Fuehrmann, Tobias

Fuentes-Mattei, Enrique

Fujihara, Hisako

Fujii, Hiroaki

Fujii, Tomomi

Fujii, Yoshihiro

Fujimori, Akira

Fujimori, Ko

Fujimoto, Ryo

Fujita, Mikako

Fujita, Mitsugu

Fujita, Satoshi

Fujita, Tetsuji

Fujita, Yu

Fujita, Yuki

Fujitani, Yoshio

Fujiwara, Naoto

Fujiyama, Kazuhito

Fukuchi, Satoshi

Fukuda, Machiko

Fukui, Hirokazu

Fukunaga, Kenji

Fukunaga, Tsukasa

Fukushima, Atsushi

Fukushima, Tsuyoshi

Fukuta, Shiro

Fuller, Bryan

Funaba, Masayuki

Funder, John

Funel, Niccola

Funk, Richard

Furler, Robert L.

Furukawa, Tatsuhiko

Fusaki, Noemi
Futakuchi, Mitsuru

Gaballa, Sameh

Gabelli, Sandra B.

Gaber, Timo

Gaczynska, Maria

Gaddam, Ravinder Reddy

Gafencu, Anca

Gagliano, Teresa

Gagliardi, Paolo Armando

Gahete, Manuel D.

Gahlaut, Vijay

Gaikwad, Kishor

Gaj, Thomas

Gakiere, Bertrand

Galanis, Alexis

Galateau Salle, Françoise

Galbiati, Massimo

Galderisi, Umberto

Galetti, Maricla

Gali, Himabindu

Galigniana, Mario

Galigniana, Mario D.

Gáliková, Martina

Galileo, Deni S.

Galindo, Antonio

Galitzky, Jean

Gall Troselj, Koraljka

Gallant, Nathan

Gallemí, Marçal

Galleni, Moreno

Gallo, Carmela

Galuska, Sebastian P.

Galván, Aurora

Gambino, Giorgio

Gan, Ren-You

Ganesan, Latha

Ganetzky, Rebecca D.

Gangula, Pandu

Ganguly, Anutosh

Gantenbein, Benjamin

Gao, Long

Gao, Min

Gao, Shuai

Gao, Tongchuan

Gao, Xiaolong

Gao, Yang

Gao, Yunxiang

Gaponenko, Vadim

Garaj, Vladimír

Garanto, Alejandro

Garau, Gianpiero

Garbayo, Elisa

García De Frutos, Pablo

García Garrido, José Manuel

Garcia Vaquero, Marco

Garcia, Idoia

García, Nuria

García-Caballero, Melissa 
Garcia-Calderon, Margarita

García-Estañ, Joaquín

Garcia-Estañ, Luis Perez

Garcia-Gasca, Silvia Alejandra

García-Hidalgo, Javier

García-López, Joan R.

García-Martínez, Santiago

García-Martínez, Teresa

Garcia-Oliveira, Ana Luísa

García-Ortega, Lucía

Garcia-Pardo, Javier

Garcia-Redondo, Ana Belen

Garcia-Sosa, Alfonso T.

Garcia-Velasco, Juan

García-Villalón, Angel Luis

Gard, Paul

Gardner, Paul D.

Garg, Abhishek D.

Garibotto, Valentina

Garinis, George

Garner, Angelia D.

Garner, Bianca

Garofalo, Mariangela

Garrido-Cardenas, Jose Antonio

Garrison, Jennifer L.

Garrosa, Manuel

Gascho, Dominic

Gase, Klaus

Gaspar, Diana

Gasparri, Fabio

Gasparrini, Massimiliano

Gasparyan, Armen Yuri

Gasse, Cécile

Gaszner, Balázs

Gatti, Laura

Gaudet, D.

Gaudet, Rachelle

Gauer, Stefan

Gauld, James W.

Gaumann, Andreas

Gavaia, Paulo

Gavrilenko, Tatjana

Gavrilovic, Jelena

Gawarecka, Katarzyna

Gdula-Argasinska, Joanna

Ge, Xiaodong

Geest, Bart De

Geffroy, Valerie

Geha, Sameh

Gehlert, Sebastian

Gehring, Chris

Gehrmann, Mathias

Geibel, John

Geiler-Samerotte, Kerry

Geng, Feng

Genova, Tullio

Gentile, Fabrizio

Gentile, Piergiorgio
Gentilini, Davide

Gentilucci, Luca

Georgakilas, Alexandros

George Parsons, Kimberly Suzanne

Georgiades, Savvas

Georgiev, Vasil

Georgopoulos, Nik

Geraci, Fabiana

Geraghty, Patrick

Gerbino, Andrea

Gerdol, Marco

Gergs, Ulrich

Gerhauser, Ingo

Gericke, Martin

Germain, Pierre

German, Dmitry

Gerothanassis, Ioannis P.

Gerratana, Lorenzo

Gerriets, Valerie

Gestal, Monica Cartelle

Geszke-Moritz, Małgorzata

Getty, Kelly J. K.

Geurts, Jeroen

Geurtsen, Werner

Ghaleh, Bijan

Ghandhi, Shanaz

Ghandhi, Shanaz Adi

Ghani Zadeh, Hossein

Ghatak, Arindam

Ghislat, Ghita

Ghosh, Arnab

Ghosh, Sanchita

Ghosh, Santosh

Ghoshal, Kalpana

Giachino, Claudia

Giacomazza, D.

Giacomelli, Chiara

Giacometti, Jasminka

Giakoustidis, Dimitris E.

Giampieri, Francesca

Giangrande, Angela

Gianinetti, Alberto

Giannakouros, Thomas

Giannelli, Gianluigi

Gianni, Pes

Giannotti, Marina I.

Giannotti, Marina Inés

Giansanti, Francesco

Giaume, Christian

Gibbs, Bernhard F.

Giesbertz, Pieter J.

Gil, Krzysztof

Gilding, Edward K.

Giles, Corey

Giles, Wayne R.

Gill, Martin R.

Gilles, Maud-Emmanuelle

Gillet, Reynald 
Gillevet, Patrick

Gilli, Francesca

Gimenez, Estela

Giménez, Ignacio

Giordano, Carla

Giordano, Nicola

Giotta, Livia

Giovannelli, Pia

Giovannetti, Elisa

Giovannini, Annalisa

Giovannoni, Roberto

Giraldo, Pilar

Girao, Henrique

Giraudo, Enrico

Giri, Sib Sankar

Giron, Maria Cecilia

Giroud, Maude

Giudice, Jimena

Giuliano, Michela

Giuseppina, Andreotti

Giusti, Ilaria

Gizdavic-Nikolaidis, Marija

Gkolfakis, Paraskevas

Głąbska, Dominika

Glavas-obrovac, Ljubica

Gloria, Antonio

Gluba-Brzózka, Anna

Gnocchi, Davide

Goberdhan, Deborah C. I.

Goda, Keisuke

Godos, Justyna

Godzik, Adam

Goedegebuure, Peter

Goedeke, Leigh

Goedert, James

Goel, Peeyush

Goffart, Steffi

Gogal, Robert M.

Gogulamudi, Venkateswara Reddy

Goh, Boon Chong

Gohda, Eiichi

Göhre, Vera

Gojo, Satoshi

Gok, Ozgul

Gola, Damian

Golanov, Eugene V.

Goldberg, Lynne J.

Goldhawk, Donna

Goldman, Jeremy

Goldman, Maria Helena

Goldring, Mary

Goldsmith, Jason R.

Goldstein, Peter A.

Golenhofen, Nikola

Gollahon, Lauren

Goltsov, Alexey

Golubkina, Nadezda Aleksandrovna

Golubovskaya, Vita
Golz, John F.

Gomaraschi, Monica

Gombert, Alexander

Gomes, Sónia

Gómez De Cedrón, Marta

Gomez Roldan, Maria Victoria

Gomez, Nidia

Gómez, Rodolfo

Gómez-Baena, Guadalupe

Gómez-Florit, Manuel

Gomez-Lopez, Nardhy

Gómez-Muñoz, Antonio

Gomez-Pinilla, Pedro J.

Gómez-Puertas, Paulino

Gómez-Ruiz, Santiago

Gómez-Zorita, Saioa

Gonçalves, Lidia M. D.

Gondi, Christopher S.

Gonec, Tomas

Gongol, Brendan

Gongora Castillo, Elsa

Gonkowski, Slawomir

Gonzales, Cara B.

Gonzalez Bosc, Laura V.

González, Florenci V.

Gonzalez, Gabriel

González, María Eugenia

Gonzalez, Michael J.

Gonzàlez-Duarte, Roser

Gonzalez-Escamilla, Gabriel

González-García, Ismael

González-González, Rogelio

González-Hernández, Tomás

González-Navarro, Herminia

González-Vallinas, Margarita

Gonzalez-Vicente, Agustin

Goodier, Martin R.

Gopalakrishnan, Gopan

Göpel, Sven

Gorantla, Vijay

Gordiichuk, Pavlo

Gordon, Donna

Goreham, Renee

Gorgey, Ashraf S.

Gori, Tommaso

Gorovits, Boris

Gorrell, Rebecca

Górska, Sabina

Gorski, Jeffrey

Gosselet, Fabien

Goswami, Nandu

Goto, Katsumasa

Götte, Martin

Götz, Werner

Goukassian, David

Gould, Gwyn

Gouveia, Susana

Govaere, Olivier 
Gow, Megan L.

Goyal, Rajni

Grabacka, Maja

Grabauskas, Gintautas

Grabinska, Kariona

Grabsztunowicz, Magda

Gracia-Sancho, Jordi

Gradilone, Sergio A.

Gradinaru, Andrei Cristian

Grafi, Gideon

Graler, Markus H.

Gramates, L. Sian

Gramazio, Pietro

Gramlich, Michael

Gramss, Gerhard

Grande Tovar, Carlos David

Grandemange, Stéphanie

Grant, Michael

Grässel, Susanne

Grasselli, Elena

Gray, Gordon R.

Gray, Steven

Grazul-Bilska, Anna

Greeley, Siri Atma W.

Green, Michael

Greenberg, Richard N.

Greenberg, Robert M.

Greene, Catherine

Greene, Lesley H.

Greenhalgh, Andrew David

Greenhalgh, David

Greenwood, Michael T.

Gregg, Randal

Gregor, Ingo

Gregory, Stephen

Greither, Thomas

Gremmels, Hendrik

Gresnigt, Mark S.

Gresshoff, Peter M.

Grether-Beck, Susanne

Grewal, Raji Paul

Grewal, Thomas

Griesbeck, Axel

Griffin, Robert

Grignani, Giovanni

Grijalvo, Santiago

Grimholt, Unni

Grimm, Marcus O. W.

Grinberg, Daniel

Grisoni, Francesca

Grix, Tobias

Grizzi, Fabio

Groban, Leanne

Grochans, Elzbieta

Grolli, Stefano

Grones, Peter

Grosell, Martin H.

Gross, Aaron
Großhans, Jörg

Grossi, Giancarlo

Gruca, Aleksandra

Grudnik, Przemyslaw

Gruengreiff, Kurt

Grumet, Rebecca

Grumezescu, Alexandru Mihai

Grunig, Gabriele

Gruntenko, Nataly

Grzebelus, Dariusz

Grzmil, Paweł

Grzybowska, Ewa

Grzymajlo, Krzysztof

Gu, Feng

$\mathrm{Gu}$, Yuchao

Gualtieri, Paolo

Guan, Yuan

Guardado-Calvo, Pablo

Guariglia, Emanuel

Gubbels Bupp, Melanie

Gubin, Denis

Gudey, Shyam Kumar

Gudmundsdottir, Bjarnheidur K.

Guéguen, Laurent

Guelcher, Scott A.

Guerlesquin, Francoise

Guerra, Carmen

Guerra, Iván

Guerra-Rebollo, Marta

Guerrera, M. C.

Guerrieri, Emilio

Guha, Prajna P.

Guha, Sonia

Guhathakurta, Subhrangshu

Guibert, Christelle

Guiliani, Nicolas

Guillermet Guibert, Julie

Guinane, Caitriona

Guiot, Caterina

Guix, Ester Ballana

Guizzetti, Marina

Gullberg, Donald

Gulumian, Mary

Gundamaraju, Rohit

Gunderson, Mark P.

Gundogdu, Ozan

Günes, Cagatay

Gunness, Purnima

Günther, Andreas

Guo, Chih-Hung

Guo, Longbiao

Guo, Qiuquan

Guo, Shengfeng

Guo, Weimin

Guo, Yin

Gupta, Dorin

Gupta, Sheeba Varghese

Gupta, Shuchi 
Gupta, Vijayalaxmi

Gupta-Rossi, Neetu

Gurevich, Vsevolod V.

Gurruchaga, Mariló

Gururani, Mayank Anand

Gust, Ronald

Gutiérrez, Norma C.

Gutiérrez-Juárez, Roger

Gutleb, Arno

Gutschner, Tony

Guy, Charles L.

Guzik, Urszula

H Shirvani, Arash

H. Bailey, William

Ha, Hyunjung

Ha, Jungmin

Ha, Patrick

Haarmann-Stemmann, Thomas

Haase, Hajo

Hachiya, Tsuyoshi

Hack, Ethan

Hada, Megumi

Hadden, Kyle

Hadjiargyrou, Michael

Hadjikakou, Sotiris

Hadjipavlou-Litina, Dimitra

Hadler-Olsen, Elin

Haendler, Bernard

Haffner-Luntzer, Melanie

Häfner, Norman

Hagland, Hanne R.

Hagstrom, Stephanie A.

Hailfinger, Stephan

Hajjar, Katherine

Hakkinen, Lari

Halatsch, Marc-Eric

Haldar, Sumanto

Hales, Karen $\mathrm{H}$.

Halford, Michael

Hallows, Kenneth

Ham, Daniel

Hamacher, Jürg

Hamanishi, Junzo

Hambley, Trevor

Hamblin, Michael

Hametner, Bernhard

Hamilton, Duane H.

Hamm-Alvarez, Sarah

Hamm-Alvarez, Sarah F.

Hamon, Morgan

Hamrick, Mark W.

Han, Byung Woo

Han, Dong

Han, Dong-Wook

Han, Hong-Yu

Han, Jaehong

Han, Pingping

Han, Qifeng
Han, Xiaoyuan

Hanai, Jun-ichi

Hanaoka, Mitsumasa

Handzlik, Jadwiga

Hanif, Muhammad

Hankinson, Oliver

Hano, Christophe

Hano, Takuzo

Hanson, Johannes

Hanss, Michel

Hantusch, Brigitte

Hao, Jiaqing

Haque, Inamul

Hardiman, Gerard

Harding, Katharine

Hardingham, Jennifer E.

Hardwick, James P.

Hardy, John

Hardy, John G.

Hardy, Rowan

Hargreaves, Iain

Hargreaves, Iain P.

Hariharan, Seethalakshmi

Harijith, Anantha

Harmanci, Arif Ozgun

Harn, Horng-Jyh

Haro, Diego

Harp, Tyler

Harper, Alan

Harrington, Charles

Harris, Andrew R.

Harris, Edward N.

Harris, William

Harrison, Jonathan S.

Hartman, Mariusz

Hartman, Matthew

Harvey, Adam

Harvey, Alexandra

Harvey, Kirsten

Harvill, Eric

Hasanuzzaman, Mirza

Hasegawa, Morifumi

Hashimoto, Makoto

Hashimoto, Masayuki

Haspel, Nurit

Hassan, Mohamed

Hassan, Sherif T. S.

Hatami, Asa

Hatano, Noriyuki

Hatano, Tsutomu

Hatt, Séverin

Hattori, Toshio

Hatziantoniou, Sophia

Haudecoeur, Romain

Haumann, Michael

Haurani, Mounir J.

Hauser, Peter

Hausman, Gary J. 
Hausner, Georg

Hawinkels, Lukas

Hayakawa, Tohru

Hayakawa, Yoku

Hayashi, Yohei

Haynes, Nicole M.

Hazak, Ora

Hazawa, Masaharu

He, Aina

$\mathrm{He}$, Chunbo

$\mathrm{He}$, Fei

He, Feng

He, Guanglong

He, Hailun

He, Jing

He, Xiaobo

Heaphy, Christopher

Heath, Timothy

Heberden, Christine

Hebert, Terry

Heckathorn, Scott A.

Hegele, Robert A.

Heggermont, Ward

Heggeset, Tonje Marita Bjerkan

Hegyi, Péter

Hehlgans, Stephanie

Hehlmann, Rüdiger

Heiker, John T.

Heinbockel, Thomas

Hejatko, Jan

Helaly, Soleiman

Helle, Francois

Heller, Gerwin

Heller, Janosch

Hellmann, Jason L.

Hellström, Mats

Hellweg, Christine

Helm, Richard

Helmy, Adel

Henagan, Tara M.

Hendriks, Lizza

Henen, Morkos A.

Hengesbach, Martin

Henkel, Janin

Hennequin, Claire

Henriksson, Marie

Henrique, Rui

Heo, Yong-Seok

Her, Lu-Shiun

Herfindal, Lars

Herington, Jennifer L.

Hermey, Guido

Hernández Ledesma, Blanca

Hernández, M. Luisa

Hernandez, Maria

Hernández-Hernández, Ángel

Herold, Nikolas

Herr, Deron Raymond
Herránz, Héctor

Herrero-Beaumont, Gabriel

Herrmann, Harald

Hertegård, Stellan

Herten, Monika

Hess, Angela

Hetman, Michal

Heumann, Rolf

Heusinkveld, Harm

Hevey, Rachel

Hewett, Sandra

Heymann, Dominique

Higashida, Haruhiro

Hihara, Yukako

Hildenbrand, Georg

Hildreth, Blake

Hilker, Monika

Hillman, A. Robert

Hills, Ronald

Himeno, Hyouta

Himoto, Takashi

Hinderlich, Stephan

Hindra, Hindra

Hinds Jr., Terry D.

Hinojosa, Silvia

Hiraga, Toru

Hirakawa, Hidehiko

Hirano, Katsuya

Hirano, Shigeru

Hirasaka, Katsuya

Hirasawa, Noriyasu

Hirata, Yoko

Hirata-Tsuchiya, Shizu

Hirayama, Takashi

Hiroi, Noboru

Hirsch, Mark A.

Hirschi, Kendal D.

Hiruma, Kei

Ho, Honhing

Ho, Mengfei

Ho, Roger

Ho, Roger Chun Man

Ho, Shin-Lon

Ho, Vincent

Ho, Wen-Fu

Ho, Wing Shing

Ho, Yi-Cheng

Hoang, Nam V.

Hoang-Vu, Cuong

Höckner, Martina

Hodge, Allison

Hodges, Lynette

Hodson, Martin

Hoffman, Nolan J.

Hoffmann, Klaus

Hofmann, Johann

Høivik, Erling Andre

Hojjat-Farsangi, Mohammad 
Hojo, Hironori

Holalu, Srinidhi

Holan, Vladimir

Holecek, Milan

Holefors, Anna

Hollenbach, Marcus

Hollomon, Mario G.

Holmes, Dawn E.

Holmøy, Trygve

Holst, Otto

Holt, Scott M.

Holvik, Kristin

Holzel, Christina

Holzmann, Klaus

Homa, Joanna

Homma, Hiroshi

Homma, Tetsuya

Hong, Qiuting

Hong, Soyon

Hong, Yonggeun

Hooda, Jagmohan

Hooykaas, Paul J. J.

Hopkins, Samantha

Horejs-Hoeck, Jutta

Horhat, Florin George

Hori, Toshiyuki

Hori, Yuuichi

Horino, Yoshikazu

Horn, Patrick J.

Hornsveld, Marten

Horowitz, Mia

Horstkorte, Rüdiger

Horvath, David P.

Horvath, Dragos

Horvath, Robert

Hosek, Petr

Hosgood, Sarah

Hoshi, Akihiko

Hoshiba, Takashi

Hoshino, Masaru

Hoskins, Clare

Hosohata, Keiko

Hosokawa, Yoshitaka

Hosório, Hugo

Hossain, Mokarram

Hoštálková, Anna

Hotta, Jun-ichi

Hotta, Yoshihiro

Hou, Anfu

Hou, Chien-Wei

Hou, Harvey

Hou, Panpan

Houen, Gunnar

Howell, Viive

Hoy, Andrew

Hoyos-Villegas, Valerio

Hozbor, Daniela

Hrabal, Richard
Hrelia, Patrizia

Hsia, Shih-Min

Hsiao, George

Hsieh, Chengying

Hsieh, Chia-Chien

Hsieh, Hsi-Lung

Hsieh, Tusty-Juan

Hsu, Chung Y.

Hsu, Fang Rong

Hsu, Fei-Ting

Hsu, Kuo-Sheng

Hsu, Ren-Jun

Hsu, Shang-Te Danny

Hsu, Sung-Po

Hsu, Todd

Hsu, Tsung-I

Hsu, Yi-Chiung

Hsueh, Yi-Huang

$\mathrm{Hu}$, Guang

$\mathrm{Hu}$, Guanggan

$\mathrm{Hu}$, Helen

$\mathrm{Hu}$, Jianying

$\mathrm{Hu}$, Jin-Jia

$\mathrm{Hu}$, Marian Y.

$\mathrm{Hu}$, Min

$\mathrm{Hu}$, Qiwen

$\mathrm{Hu}$, Zhenbin

Huang, Chunfa

Huang, Guan-Jhong

Huang, Hao

Huang, Huey-Chun

Huang, Jason C.

Huang, Jian

Huang, Lei

Huang, Lei (Helen)

Huang, Nai-Kuei

Huang, Rongfeng

Huang, Shu-Hung

Huang, Wei

Huang, Wen-Lii

Huang, Wenrui

Huang, Xiaohu

Huang, Yadong

Huang, Ying-Hsien

Huang, Yu-Chuen

Huang, Yung-Fen

Huber, Heinrich

Huber, Stephan M.

Huber, Veronica

Hubková, Beáta

Hübner, Christian

Huchzermeyer, Bernhard

Hudzieczek, Vojtech

Hui, Kwai Fung

Hui-Yuen, Joyce S.

Humbeck, Klaus

Humphries, Brock

Humphries, Jonathan D. 
Hung, Shih-Ya

Hunter, Richard G.

Hunter, Robert P.

Huppi, Konrad

Hur, YoonKang

Hura, Katarzyna

Hura, Tomasz

Hurley, Margaret

Huss, Fredrik

Hussain, Mulazim

Hussein, Waleed

Hutchinson, Edward

Hutchinson, Edward C.

Hutchison, Robert E.

Hutmacher, Dietmar W.

Hwa Soung, Young

Hwang, In Koo

Hwang, Pung Pung

Hwang, Yongsung

Hyndman, Kelly Anne

Hyun, Kyung-Yae

Hyun, Sang-Hwan

I Gossmann, Toni

Iaccino, Enrico

Iacobazzi, Vito

Iacoviello, Massimo

Iannuzzi, Clara

Iatsenko, Igor

Ibarra Molero, Beatriz

Ibrahim, Hussameldin

Ibrahim, Sulaiman Sadi

Ichihara, Sahoko

Ichihashi, Masamitsu

Ichitani, Katsuyuki

Ide, Kazuki

Ido, Yasuo

Ientile, Riccardo

Ieraci, Alessandro

Igaz, Peter

Iglesias-Fernández, Raquel

Iglesias-Linares, Alejandro

Iguchi, Taisen

Iida, Hiroshi

Iijima, Noriaki

Iizuka, Katsumi

Ikarashi, Nobutomo

Ikeda, Masahiro

Ikegaya, Naoki

Ikeguchi, Masamichi

Ikehara, Kenji

Ikeshima-Kataoka, Hiroko

Ikura, Yoshihiro

Ikushiro, Shin-ichi

Ikuta, Tetsuro

Ilagan, Ma. Xenia G.

Ilardi, Gennaro

Ilavenil, Soundharrajan

Ilies, Marc A.
Ilieva, Zhenya

Ilmer, Matthias

Ilyas, Azhar

Im, Chang-Nim

Imai, Hiroo

Imai, Toshio

Imai, Yoichi

Imamoto, Naoko

Imamoto, Yasushi

Imbert, Véronique

Imbrici, Paola

Imhof, Petra

Imig, John

Imokawa, Genji

Impellizeri, Joseph

Imperlini, Esther

Ina, Kenji

Inaba, Hiroshi

Inada, Natalia

Inagaki, Takeshi

Inamura, Kentaro

Incitti, Tania

Indiveri, Cesare

Ingre, Caroline

Iniesta, Raquel

Inoue, Atsuko

Inoue, Kazushi

Inui, Akio

Inui, Masafumi

Iohara, Daisuke

Iommarini, Luisa

Iordanskii, Alexey

Iorio, Ronald M.

Iorizzo, Massimo

Iotti, Mirco

Iqbal, Khalid

Iqbal, Tariq $\mathrm{H}$.

Iraci, Nunzio

Irato, Paola

Iriepa Canalda, Isabel

Irimura, Tatsuro

Irvine, Scott A.

Irving, Thomas C.

Isaji, Shuji

Isaza Martínez, José Hipólito

Isemura, Mamoru

Isgaard, Jörgen

Ishibashi, Kenichi

Ishida, Takashi

Ishii, Tetsuro

Ishii, Yumiko

Ishikawa, Yasuyuki

Ishimaru, Naozumi

Ishimoto, Takatsugu

Ishizaki, Takuma

Islam, Md Soriful

Isman, Murray B.

Isoda, Katsuhiro 
Isola, Gaetano

Isozaki, Takeo

Itahana, Koji

Itami, Tashiaki

Ito, Naoki

Ito, Shosuke

Ito, Takuhiro

Ito, Toshiro

Ito, Yasuhiro

Ito, Yuji

Itoh, Tohru

Itokazu, Yutaka

Itou, Junji

Ivancic Santek, Mirela

Ivanov, Alexander G.

Ivanov, Alexander V.

Ivanov, Alexander Vladimirovich

Ivanov, Andrey

Ivanova, Magdalena I.

Iwai, Atsushi

Iwaizumi, Masakazu G.

Iwamoto, Hiroyuki

Iwamoto, Shotaro

Iwata, Junichi

Iwona, Niedzielska

Izasa, Hisashi

Izawa, Takashi

Izawa, Takeshi

Izuhara, Kenji

Jaaro-Peled, Hanna

Jablonska, Ewa

Jablonska, Karolina

Jackson, Christopher

Jacob, Francis

Jacobs, Delphine

Jacobs, Elizabeth R.

Jacomin, Anne-Claire

Jacques, Danielle

Jacquin, Lisa

Jadavji, Nafisa M.

Jadhav, Gopal P.

Jaeschke, Hartmut

Jaffar, Zeina

Jagannathan, Vidhya

Jain, Ashish

Jain, Vaibhav

Jaing, Tang Her

Jaiswal, Amit K.

Jaiswal, Ashvin R.

Jaiswal, J. K.

Jaligot, Estelle

James, William

Jamwal, Rohitash

Janczar, Szymon

Janczarek, Monica

Janda, Elzbieta

Janda, Tibor

Janetka, James
Janevska, Slavica

Jang, Jinah

Jang, Sung-Wuk

Janga, Madhusudhana

Jänig, Wilfrid

Janikowska, Grażyna

Janiuk, Izabela

Janowiak, Blythe E.

Janowski, Miroslaw

Jansen, Gerrit

Jansen, Rick

Jansky, Shelley

Januchowski, Radoslaw

Janusz, Grzegorz

Jaradat, Abdullah

Jaradat, Nidal

Jarajapu, Yagna

Jara-Palacios, María José

Jardin, Isaac

Jardine, Kolby

Jarret, Robert L.

Jarstfer, Michael Bruce

Jarząb, Barbara

Jat, Parmjit S.

Jaumot, Joaquim

Jaurand, Marie-Claude

Jayant, Rahul Dev

Jayaram, Lata

Jayasinghe, Suwan

Jazirehi, Ali R.

Jelena, Krstic

Jellinger, Kurt A.

Jena, Prasant Kumar

Jeng, Jiiang-Huei

Jenkins, Paul

Jenkins, Samir V.

Jensen, Eric D.

Jensen, Lars Henrik

Jeon, Byeong Hwa

Jeong, Daewon

Jeong, Hyun Young

Jeong, Jinyoung

Jeong, Keun-Yeong

Jeong, Seon-Yong

Jeppe, Emmersen

Jerebtsova, Marina

Jeremy, Teoh Yuen Chun

Jerome, De Ruyck

Jeschek, Markus

Jeschke, Marc G.

Jeschke, Udo

Jessen, Niels

Jetten, Anton

Jetybayev, Ilyas Yerkinovich

Jeung, Eui-Bae

Jeynes, Charlie

Jeziskova, Ivana

Jha, Amit 
Jha, Mithilesh

Jha, Sudhakar

Ji, Peng

Ji, Tianjiao

JI, Yuelong

Jiang, Chen Chen

Jiang, Jianxiong

Jiang, Qiu-Xing

Jiang, Shu-Ye

Jiang, Sizun

Jiang, Tao

Jiang, Xinyin

Jiang, Xiqian

Jiao, Chen

Jiao, Yinping

Jimbo, Mitsuru

Jiménez-Alemán, Guillermo

Jimenez-Rondan, Felix

Jimi, Eijiro

Jin, Dong-Hoon

Jin, Fan

Jin, Jiefu

Jin, Xin

Jin, Young Woo

Jindra, Marek

Jindrich, Jindrich

Jinping, Zhao

Jirkovská, Anna

Jirkovsky, Eduard

Jo, Young Suk

João, Benevides

Job, Dominique

Jochum, Christoph

John, Rohan

Johnen, Georg

Johnson, Andrew D.

Johnson, Daniel E.

Johnson, Erik

Johnson, Jay E.

Johnson, Kim

Johnson, Lance A.

Johnson, Thomas V.

Johnsson, Martin

Johnston, Carol

Johnstone, Scott

Johnstone, Scott R.

Joles, Jaap

Jonassen, Christine

Jones, Aleck

Jones, Malcolm

Jones, Mark G.

Jones, Meriel

Jonušienè, Violeta

Joo, Kyeung Min

Joo, Yuyoung

Jordi, Gomez

Jørgensen, Anders Palmstrøm

Jørgensen, Hans Jørgen Lyngs
Jørgensen, Kirsten

Josef, Kapfhammer

Joseph, Susan

Joshi, Bharat

Joubert, Olivier

Jouk, Pierre Simon

Joung, Hyou-Arm

Jovanović, Aleksandar

Jovanović, Dragana

Jovanovic-Santa, Suzana

Jóźwicki, Wojciech

Juarranz, Ángeles

Jubeli, Emile

Juhas, Stefan

Julve, Josep

Jung, Chol-Hee

Jung, Ki-Hong

Jung, Klaus

Jung, Sung-Chul

Jurczyk, Barbara

Jurenka, Russell

Jurikova, Tunde

Juriloff, Diana M.

Just, Nathalie

Just, Steffen

K Song, Moon

Kabała-Dzik, Agata

Kabbage, Mehdi

Kabil, Omer

Kačarević, Željka Perić

Kachamakova-Trojanowska, Neli

Kaczmarek, Beata

Kaczorek, Ewa

Kadam, Suhas

Kaddoumi, Amal

Kaddour, Hussein

Kadimisetty, Karteek

Kadokawa, Jun-ichi

Kagota, Satomi

Kai, Mihoko

Kaina, Bernd

Kajava, Andrey

Kajiya, Hiroshi

Kajiya, Mikihito

Kakehashi, Anna

Kakinen, Aleksandr

Kakumanu, Akshay

Kalani, Komal

Kalatzis, Vasiliki

Kalayda, Ganna

Kalber, Tammy

Kaldis, Philipp

Kale, Abhijit

Kalhapure, Rahul

Kaliniewicz, Zdzisław

Kallergi, Galatea

Kallikourdis, Marinos

Kalogirou, Charis 
Kalthoff, Holger

Kaluderovic, Goran

Kam, Yiu Wing

Kamagata, Kiyoto

Kamal, Abu Hena Mostafa

Kamath, Divya

Kamato, Danielle

Kaminaka, Hironori

Kaminski, Rafal

Kamitori, Shigehiro

Kamolz, Lars

Kanakkanthara, Arun

Kanakry, Christopher G.

Kanasak, Keizo

Kanazawa, Masato

Kanda, Teru

Kandimalla, Raju

Kanekiyo, Takahisa

Kaneko, Gen

Kanematsu, Hideyuki

Kang, Chen

Kang, Chul Hee

Kang, CongBao

Kang, Hee Y.

Kang, Sang Sun

Kang, Sinyoung

Kang, Tae-Hong

Kang, Wei

Kang, Young-Hee

Kannan, Ram

Kanojia, Deepak

Kant, Surya

Kanzaki, Hiroyuki

Kao, Shu-Huei

Kaplan, Shauna

Kaplinsky, Nicholas J.

Kappachery, Sajeesh

Kaput, Jim

Karabagias, Ioannis

Karafiloglou, Padeleimon

Karagöz, Elif

Karakasidis, T. E.

Karaki, Shin-ichiro

Karakoula, Katherine

Karakülah, Gökhan

Karan M, Shah

Kararigas, Georgios

Karasiński, Janusz

Kardos, József

Kärenlampi, Sirpa

Karganova, Galina G.

Kari, Leif

Karkanis, Anestis

Karlic, Heidrun

Karlsson, Erik

Karmakar, Mausita

Karmakar, Partha

Karmazyn, Morris
Karpiński, Tomasz M.

Kasahara, Ryushiro D.

Kaschula, Catherine H.

Kasetti, Ramesh

Kashiwagi, Shinichiro

Kashtalap, Vasily

Kashuba, Elena

Kasinath, Balakuntalam S.

Kasoju, Naresh

Kasparkova, Jana

Kast, Richard Eric

Kasten-Jolly, Jane

Katada, Kazuhiro

Kataoka, Naoyuki

Katarivas Levy, Galit

Katayama, Kentaro

Kateřina, Schwarzerová

Kathuria, Himanshu

Kato, J.

Kato, Koichi

Kato, So

Kato, Takamitsu A.

Kato, Yasumasa

Katsantonis, Dimitrios

Kaul, Marcus

Kaunas, Roland R.

Kauppinen, Anu

Kaur, Amanpreet

Kaur, Jasmine

Kavita, Kumari

Kawabata, Kohsuke

Kawabata, Kyuichi

Kawabe, Akira

Kawaguchi, Shin-ichi

Kawai, Takayuki

Kawakami, Hiroshi

Kawakami, Susumu

Kawanami, Daiji

Kawano, Kouichiro

Kawasaki, Hideki

Kawase, Tomoyuki

Kawata, Kazumi

Kawauchi, Takeshi

Kay, Gemma

Kayano, Shin-ichi

Kayser, Klaus

Kazama, Tomohiko

Kazeto, Yukinori

Kazmi, Faraz

Ke, Liang-Yin

$\mathrm{Ke}$, Youqiang

Keely, Simon

Kefalov, Vladimir

Keil, Kimberly

Keller, Andreas

Kelley, Darshan

Kelley, Eric

Kello, Martin 
Kelly, Michy P.

Kemp, Bruce E.

Kemp, Michael G.

Kempińska-Podhorodecka, Agnieszka D.

Kenchanmane Raju, Sunil Kumar

Kendig, Michael

Kenealey, Jason

Kenichi, Sakurai

Keniya, Mikhail V.

Kennedy, David

Kenny, Hilary A.

Kerwin, Rachel E.

Kerz, Thomas

Kessel, David

Kevadiya, Bhavesh

Khairullina, Veronika

Khalil, Christian

Khalil, Zeinab

Khan, Atif Ali

Khan, Mohd Parvez

Khan, Mohsin

Khan, Muhammad

Khapli, Sachin

Kharbanda, Kusum K.

Kharissov, Boris Ildusovich

Kharkar, Prathamesh

Khasawneh, Fadi

Khatodia, Surender

Khatri, Bhuwan

Khatri, Kshitij

Khattab, Muhammad

Khazaei, Hamid

Khlebtsov, Nikolai G.

Khoda, Bashir

Khodanovich, Marina

Khoshmanesh, Khashayar

Khotimchenko, Maxim Y.

Khuder, Sadik A.

Khudyakov, Jane

Khurshid, Zohaib

Ki, Sung Hwan

Kiaei, Mahmoud

Kianifariz, Mahnaz

Kibriya, Muhammed

Kiefer, Friedemann

Kiełbowicz-Matuk, Agnieszka

Kielbus, Andrzej

Kiełczykowska, Małgorzata

Kieliszek, M.

Kieliszek, Marek

Kielland-Brandt, Morten

Kienesberger, Petra

Kietzmann, Thomas

Kikis, Elise A.

Kikowska, Małgorzata

Kikuta, Shingo

Kilari, Sreenivasulu

Kilinc, Devrim
Kiliszek, Agnieszka

Kilpatrick, Laura

Kim, AeRang

Kim, Bhumsoo

Kim, Byung-Soo

Kim, Chang Kil

Kim, Chang Wook

Kim, Cheorl-Ho

Kim, Choel

Kim, Dong Joon

Kim, Dongin

Kim, Dong-Seon

Kim, Donguk

Kim, Hangun

Kim, Hoon

Kim, Hoy-Taek

Kim, Hun Sik

Kim, Hye One

Kim, Hyesun

Kim, Hyeung-Rak

Kim, Hyounju

Kim, Hyun Ah

Kim, Hyun Joon

Kim, Hyun Soon

Kim, Hyung Sik

Kim, Hyung-Joon

Kim, In-Jung

Kim, InKyeom

Kim, J. Julie

Kim, Jeong-Rae

Kim, Jin-Bom

Kim, Jin-Wook

Kim, Joungmok

Kim, Ki Hyun

Kim, Kyoung Soo

Kim, Kyounghyun

Kim, Kyung-Min

Kim, Kyung-su

Kim, Moon Ki

Kim, Nam Deuk

Kim, Nam-Jung

Kim, Sang-We

Kim, Seongho

Kim, Sunggil

Kim, Sung-Hoon

Kim, Sung-Kun

Kim, Tae Il

Kim, Tae-Hyung

Kim, Taewan

Kim, Weon

Kim, Woojin

Kim, Yangmee

Kim, Yong-mi

Kim, Youngsoo

Kim, Yun Kyung

Kim, Yun-bae

Kimber-Trojnar, Żaneta

Kimbrel, Erin 
Kimler, Bruce

Kimura, Kei

Kimura, Motoko

Kimura, Seisuke

Kindy, Mark

King-Lyons, Natalie D.

Kirberger, Michael

Kirillova, Alina

Kirsch, Thorsten

Kirschnek, Susanne

Kirton, Stewart B.

Kishaba, Tomoo

Kishi, Takuya

Kishikawa, Naoya

Kishimoto, Yoshimi

Kiskova, Terezia

Kiss, Anna

Kiss, John

Kiss, Robert S.

Kitagawa, Seiichi

Kitamura, Naoki

Kitamura, Takanori

Kitaoka, Satoshi

Kitatani, Kazuyuki

Kitchen, Philip

Kitson, Matthew T.

Kittaka, Atsushi

Kiyan, Yulia

Kjøbsted, Rasmus

Klaassen, Curtis D.

Klajner-Maculewicz, Barbara

Klajnert-Maculewicz, Barbara

Klampfer, Lidija

Klapdor, Rüdiger

Klar, Agnes

Klassen, Roland

Klaus, Susanne

Kleczkowski, Leszek A.

Klein, Axel

Klein, Janet D.

Kleine, Tatjana

Klementieva, Oxana

Kleppe, Lene

Klettner, Alexa

Klimek, Katarzyna

Klokov, Dmitry Y.

Klontzas, Michael

Klose, Holger

Klose, Johannes

Klotz, James

Klugbauer, Norbert

Klusa, Vija

Kluza, Jérome

Klychnikov, Oleg

Knap, Narcyz

Knecht, Hans

Knepper, Janice

Knight, David
Knight, Jason S.

Knight, Vijaya

Knipper, Johanna A.

Knoch, Eva

Knoell, Daren

Knoell, Ralph

Knott, Rachel

Knox, Paul

Ko, Jane L.

Ko, Jiunn- Liang

Kobayashi, Jun

Kobayashi, Natsuko I.

Kobayashi, Satoru

Kobayashi, Yayoi

Kobayashi, Yoshiro

Kobayashi, Yutaro

Kobeissy, Firas H.

Köbel, Martin

Koch, Alexander

Koch, Stefan

Koch, Wojciech

Kocot, Joanna

Kodama, Hiroaki

Koepke, Tyson

Koga, Jun-ichiro

Kogashiwa, Yasunao

Koh, Cho Yeow

Koh, Gar Yee

Koh, Ho-Jin

Köhl, Gudrun

Koibuchi, Noriyuki

Koike, Kenzo

Koivisto, Ari

Koizume, Shiro

Kojima, Chojiro

Kojima, Masami

Kojima, Shihoko

Kojima-Yuasa, Akiko

Kok, Dieuwertje

Kok, Wouter

Kokabu, Shoichiro

Kolbe, Ludger

Kolesárová, Adriana

Kolettas, Evangelos

Koller, Gabriele

Kolluri, Siva Kumar

Kolluru, Gopi

Kolmas, Joanna

Kolodych, Sergii

Kolosov, Dennis

Kolosov, Peter

Kolukisaoglu, Üner

Komatsu, Ken

Komine, Mayumi

Komis, George

Kommineni, Vally

Kommireddy, Vasu

Konar, Arkaprabha 
Kondo, Hidehiro

Kondo, Tatsuya

Kondratieff, Boris

Koneru, Bhuvaneswari

Kones, Richard

Kong, Yimeng

König, Daniel

Koninckx, Philippe R.

Konishi, Masaaki

Konno, Hiroyuki

Konopka-Postupolska, Dorota

Konstandin, Mathias

Konstantinov, Spiro M.

Kontny, Udo

Kontogiannatos, Dimitrios

Kontoravdi, Cleo

Koo, Heebeom

Kopecki, Zlatko

Kopecký, Vladimír

Kopel, Pavel

Koppe, Laetitia

Korać, Petra

Korasick, David A.

Korenjak, Michael

Korfei, Martina

Korkaya, Hasan

Korkina, Ludmila G.

Kornelyuk, Alexander

Korou, Laskarina-Maria

Korsching, Eberhard

Korte, Cassandra

Koschella, Andreas

Kosmas, Constantine E.

Kosova, Klara

Kostakis, Ioannis

Kosters, Astrid

Kostyuk, Vladimir A.

Kotsikorou, Evangelia

Kotsinas, Athanassios

Kotta-Loizou, Ioly

Kotula-Balak, Malgorzata

Kotyla, Przemyslaw J.

Koubassova, Natalia

Koul, Hari

Koulen, Peter

Koupenova, Milka

Kourkoumelis, Nikolaos

Kousik, Chandrasekar S.

Koutelidakis, Antonios E.

Kovac, Stjepana

Kovacs, Richard

Koval, Alexey

Koval, Michael

Kovbasnjuk, Olga

Kovermann, Michael

Kovinich, Nik

Kowalczuk, Marek

Kowalska, Katarzyna
Kowtharapu, Bhavani S

Koya, Daisuke

Koyama, Ryuta

Koyama, Yu

Kozaki, Akiko

Koziak, Katarzyna

Kozieł, Edmund

Kozikowski, Alan

Kozlov, Konstantin

Kozlova, Elena

Kozlowski, Michael R.

Kraemer, Brian C.

Kraft, Robert

Kraic, Jan

Krajewska-Włodarczyk, Magdalena

Kramer, Phillip

Kramnik, Igor

Kranz, Stefan

Krasowska, Anna

Krause, Günter

Krebs, Joachim

Kren, Vladimir

Krenács, Tibor

Krenn, Veit T.

Krenz, Björn

Kreyling, Wolfgang

Krick, Stefanie

Kriebardis, Anastasios G.

Krishnan, Natraj

Krishnan, Sanalkumar

Kristensen, Lasse Sommer

Kristensen, Mie

Krogan, Naden T.

Król, Ewelina

Kroncke, Brett M.

Kropski, Jonathan A.

Krycer, James

Kschischo, Maik

$\mathrm{Ku}$, Kang-Mo

$\mathrm{Ku}$, Seung-Yup

Kuan, Yu-Hsiang

Kubaczka, Caroline

Kubala, Szymon

Kubatka, Peter

Kubíček, Vojtěch

Kubina, Robert

Kubinova, Sarka

Kubiński, Konrad

Kubista, Helmut

Kubo, Atsushi

Kubo, Nakao

Kubo, Takanori

Kubo, Toshio

Kubota, Toshiaki

Kuca, Kamil

Kuçi, Selim

Kucinska-Lipka, Justyna

Kucuk, Israfil 
Kucukkal, Tugba

Kudłak, Błażej

Kudo, Yasusei

Kudryasheva, Nadezhda S.

Kudryashova, Tatiana V.

Kuebler, Dan

Kuehl, Michael

Kues, Wilfried A.

Kuhla, Angela

Kuhlmann, Markus

Kuhn, Andreas

Kühnlein, Ronald

Kujan, Omar

Kujawska, Małgorzata

Kukimoto, Iwao

Kukley, Maria

Kukula-Koch, Wirginia

Kulawiak, Bogusz

Kulbacka, Julita

Kulma, Anna

Kulus, Dariusz

Kumamoto, Eiichi

Kumar, Amit

Kumar, Binod

Kumar, Devender

Kumar, Gaurav

Kumar, Gokhlesh

Kumar, Manoj

Kumar, Manu

Kumar, Narender

Kumar, Pradeep

Kumar, Rajiv

Kumar, Sathish

Kumar, Sujeet

Kumar, Sunil

Kumar, Sushil

Kumar, Ujendra

Kumar, Vasanth

Kumari, Asha

Kumasawa, Keiichi

Kumi-Diaka, James

Kundu, Aishwarya

Kundu, Parag

Kunej, Tanja

Kung, Yu-Chun

Kunioka, Masao

Kunz, Meik

Kunz, Wolfram

Kuo, Cheng-Chin

Kuo, Lih

Kuo, Ping-Chung

Kuo, Po-Lin

Kuo, Tzong-Fu

Kupai, Krisztina

Kupčinskas, Juozas

Kupcova Skalnikova, Helena

Kuppusamy, Maniselvan

Kurakula, Kondababu
Kurapati, Rajendra

Kurczynska, Ewa

Kurdowska, Anna K.

Kurgan, Lukasz

Kurien, Biji Theyilamannil

Kuroda, Masashi

Kurokawa, Tetsuji

Kurreck, Jens

Kurrikoff, Kaido

Kurschus, Florian

Kurth, Ina

Kuryk, Lukasz

Kurylowicz, Alina

Kuryłowicz, Alina

Kurz, Jacqueline LaRose

Kurzyńska-Kokorniak, Anna

Kus, Piotr

Kus-Liśkiewicz, Małgorzata

Kusmartsev, Sergei

Kusters, Ilja

Kusuma, Gina

Kuter, Katarzyna

Kuzmin, Ivan

Kuznetsova, Irina M.

Kuźnicki, Jacek

Kuźnik, Nikodem

Kuzuya, Akinori

Kwak, Brenda R.

Kwakowsky, Andrea

Kweon, Oh-Kyeong

Kwiatkowska, Katarzyna

Kwiecien, Jacek M.

Kwok, Seung-Ki

Kwon, Jae-Sung

Kwon, Kyoung Ja

Kwon, Tae-Hwan

Kwon, Tae-Yub

Kwon, Young M.

Kwong, Joseph

Kyle, Stuart

Kyoko, Oka

La Barbera, Giorgia

La Ferla, Barbara

La Regina, Giuseppe

La Rocca, Renato V.

La Rosa, Valentina Lucia

La Russa, Daniele

LaBean, Thomas H.

Labruna, Giuseppe

Lacal, Juan Carlos

Lacham-Kaplan, Orly

Lachowiec, Jennifer

Lacis, Gunars

Lacomme, Christophe

Lafarga, Tomas

Laforenza, Umberto

Lagali, Pamela

Lagana, Antonio Simone 
Lagger, Sabine

Lahiri, Amit

Lahiri, Amitabha

Lahiri, Tanaya

Lahooti, Hooshang

Lai, Jui-Yang

Lai, Tsung-Hsuan

Lai, Wen-Fu Thomas

Lai, Yandong

Lai, YingJu

Laimins, Laimonis

Lakatos, Boris

Lakatos, Lorant

Lam, Kwok Ho

Lam, Yuenting

Lamar, John Michael

Lamas, Jesús

Lamas, José Ramón

Lamb, David

Lamba, Doriano

Lambing, Christophe

Lambrinidis, George

Lamichhane, Purushottam

Lamichhane, Rajan

Lamina, Claudia

LaMontagne, Michael G.

Lampe, Jed

Lan, Lan

Lan, Ping

Lan, Yuan-Tzu

Lancelin, Jean-marc

Landreh, Michael

Landstrom, Andrew

Landström, Marene

Lang, Di

Lange, Ewa

Langford, T. Dianne

Lao, Ka Un

Lappano, Rosamaria

Laquintana, Valentino

Laranjinha, João Antonio

Laranjo, Marta

Lara-Sanchez, Agustin

Larner, Andrew

Larraneta Landa, Eneko

Larsen, Anders Christian

Lathia, Justin D.

Latorre, Juan David

Lattanzio, Rossano

Lau, Kin

Lau, Kin-Mang

Lau-Cam, Cesar A.

Laudisi, Federica

Lauf, Peter K.

Laurent, Patrick

Lauritano, Dorina

Lauro, Clotilde

Lauronen, Jouni
Lauschke, Volker

Lauth, Matthias

Lavandera, Iván

Lavogina, Darja

Layek, Buddhadev

Layh-Schmitt, Gerlinde

Lazado, C. C.

Lazár, Dušan

Lazar, Zsofia

Le Hir, Rozenn

Le Ouay, Benjamin

Le Trionnaire, Gaël

Leach, Damien A.

Leak, Rehana K.

Leanza, Luigi

Lebar, Matthew

Lebaron, Richard

LeBlanc, Roger

Lebreton, Sébastien

Lebrun, Vincent

Lecanda, Fernando

Lechanteur, Anna

Lechel, Andre

Leclercq, Guy

Lecuona, Emilia

Lecureur, Valérie

Ledda, Caterina

Leddy, Holly

Ledo, Ana

Lee, Byeong Jae

Lee, Chang Hoon

Lee, Chang Sup

Lee, ChangWoo

Lee, Che-Hsin

Lee, Cheol Koo

Lee, Cheolju

Lee, Chia-Hung

Lee, Chia-Hwa

Lee, Dae Ho

Lee, Gabsang

Lee, Gihyun

Lee, Hae-June

Lee, Huei

Lee, Hyoungseok

Lee, Jae-Ho

Lee, James

Lee, Jonghun

Lee, Joo Hyoung

Lee, Jun Hee

Lee, Kangwon

Lee, Keehoon

Lee, Li-Ting

Lee, Mingeol

Lee, Myung Koo

Lee, Sang-Han

Lee, Sanghyeob

Lee, Seong-Ryong

Lee, Shin-Da 
Lee, Soo-Hong

Lee, Su-Jun

Lee, Taesup

Lee, Tong Geon

Lee, Tzong-Shyuan

Lee, Wing-Kee

Lee, Yoon Kwang

Lee, Yunjong

Lefebvre, Hervé

Legay, Claire

Legay, Sylvain

Leggio, Lorenzo

Legler, Daniel F.

Legros, Christian

Lehotsky, Jan

Lei, Wei

Leijs, Marike M.

Leiphrakpam, Premila D.

Leitão, Ana

Leitão, Jorge H.

Leitinger, Birgit

Leitner, Alexander

Leiva-Brondo, Miguel

Lekli, István

Lembo, Giuseppe

Lemieux, KiTani

Lemke, Johannes

Lemmens-Gruber, Rosa

Lenaghan, Scott

Leng, Roger

León, Josefa

Leonard, Martin

Leonhardt, Ralf M.

Leopold, Jane A.

Lephart, Edwin

Leporatti, Stefano

Lerm, Maria

Leroux, Christine

Leroy, Baptiste

Leroy, Jo

Lescrinier, Eveline

Lesgards, Jean-François

Lesniak, Wieslawa

Leso, Veruscka

Lestavel, Sophie

Lesyk, Roman B.

Leung, Ivanhoe

Leung, Yuet-Kin

Levesque, Mitchell

Levi, Amnon

Levitskii, Sergey

Levkau, Bodo

Levy, Steven

Lev-Yadun, Simcha

Lewin, Alfred S.

Lewinska, Anna

Lewis, Jeffrey A.

Lewsey, Mathew G.
Leyva-Grado, Victor

Lézot, Frédéric

Lezza, Angela Maria Serena

Li, Chi

Li, Chunshun

Li, Dapeng

Li, Demin

Li, Fuyang

Li, Guohui

Li, Huinan

$\mathrm{Li}$, Jin

Li, Ka Wan

Li, Keshuai

Li, Liping

Li, Meichun

Li, Mei-Ling

Li, Ning

Li, Shengwen Calvin

Li, Shihao

Li, Tianhu

Li, Weijuan

Li, Wenyi

Li, Xiang

Li, Xiang-Guo

Li, Xiaohong

Li, Yan

Li, Yang

Li, Yin

Li, Zhaohui

Liagre, Bertrand

Liang, Chi-Te

Liang, Qiangrong

Liao, Albert T.

Liao, Francesca-Fang

Liao, Jiahn-Haur

Liao, Jyh-fei

Liao, Pei-Chun

Liao, Peng

Liao, Yi-Jen

Liapi, Charis

Libault, Marc

Liberale, Luca

Liby, Karen

Licausi, Francesco

Lichtstein, David

Liddell, Jeffrey R.

Lidon, Fernando

Lieberman, Scott

Liebman, Susan

Liedtke, Wolfgang

Lightfoot, Adam

Ligterink, Wilco

Lim, Bora

Lim, Edwin

Lim, Eun-Kyung

Lim, Jae Hyang

Lim, Ji-Hong

Lim, Junxian 
Lim, Khoon

Lim, Kwang-il

Lim, Soyeon

Lim, Sung-Jig

Lim, Sun-Hyung

Lim, Tae-Gyu

Lim, Yoongho

Lima, Rita

Lin, Chien-hong

Lin, Chih-Chien

Lin, Chih-Jen

Lin, Chih-Li

Lin, Dingbo

Lin, Gigin

Lin, Hai-Shu

Lin, Ho

Lin, Kwang-Huei

Lin, Liang-Tzung

Lin, Patrick P.

Lin, Pei-Hui

Lin, Shengda

Lin, Shih-Yi

Lin, Wensheng

Lin, Yiguang

Lin, Ying-Hung

Lin, Zongtao

Linder, Stig

Lindquist, Diana M.

Lindsay, Andrew J.

Lindsay, Howard

Lindsey-Boltz, Laura

Lindstrom, Jon Martin

Linge, Annett

Lingua, Guido

Linninger, Andreas A.

Lino-Neto, Teresa

Lionetti, Vincenzo

Lionetto, Maria Giulia

Lipiński, Seweryn

Lippens, Guy

Lippert, Bernhard

Lisec, Jan

Litchfield, David

Lithgow, Brian

Litofsky, Norman Scott

Litt, Amy

Liu, Aimin

Liu, Cheuk Lun

Liu, Chia-Chi

Liu, Chun-Yu

Liu, Delong

Liu, Fei

Liu, Guang-Yaw

Liu, Guo Jun

Liu, Haipei

Liu, Jia

Liu, Jian

Liu, Jianfeng
Liu, Jun

Liu, Jun-Jen

Liu, Junli

Liu, Rengyun

Liu, Shing-Hwa

Liu, Tianbo

Liu, Wei

Liu, Weizhen

Liu, Xiangsheng

Liu, Yang

Liu, Yi

Liu, Yi-Shiuan

Liu, Yongliang

Liu, Yongqing

Liu, Yu

Liu, Zhaohui

Liu, Zhihui

Liu, Zhiqiu

Liu, Zhixia

Liu-Smith, Feng

Lizardi-Mendoza, Jaime

Ljubimov, Alexander V.

Ljungdahl, Per

Llorens, Eugenio

Lloret, Ana

Lloyd, Matthew

Lluis, Frederic

Lo Muzio, Lorenzo

Lo, Amy C. Y.

Lobaccaro, Jean-Marc

Loboda, Agnieszka

Locascio, Annamaria

Locatelli, Emanuele

Locatelli, Marcello

Lochhead, Jeffrey J.

Lochman, Jan

Lockwood, Brent

Loeken, Mary R.

Loessner, Daniela

Loewendorf, Andrea I.

Löffek, Stefanie

Lognay, Georges

Loh, Kim

Lohoff, Falk

Loinard, Céline

Loiselle, Denis S.

Lombardi, Raffaella

Lonardo, Amedeo

Londei, Paola

Long, Yicheng

Longhi, Sonia

Łoniewski, Igor

Lönnberg, Tuomas

Looijenga, Leendert H. J.

Lopaschuk, Gary D.

López, Concepción

López, José

López, María L. 
Lopez, Miguel

López-Alonso, Diego

Lopez-Camarillo, Cesar

Lopez-Escamez, Jose A.

Lopez-Ferber, Miguel

Lopez-Llorca, Luis V.

Lopez-Moya, Federico

López-Tirado, Javier

Lopez-Viñas, Eduardo

LoPiero, Angela

Lopresti, Patrizia

Lora, Jorge

Lorber, Avraham

Lorberboum-Galski, Haya

Lorens, James B.

Lorenz, Kristina

Lorenzo González, Óscar

Lorenzo, Michele

Lorico, Aurelio

Losano, Gianni

Losurdo, Giuseppe

Łotocka, Barbara

Lou, Song

Loudig, Olivier D.

Loutradis, Dimitrios

Louveau, Isabelle

Loveridge, Joel

Lowe, Julie M.

Lu, Jeng-Wei

$\mathrm{Lu}$, Jing

Lu, Jun

Lu, Linchao

Lu, Mei-Chin

Lu, Quanlong

Lu, Shao-Chun

Lu, Shaoyong

$\mathrm{Lu}$, Steven

Lu, Wei-Yu

Lu, You

Lu, Zhanping

Luborsky, Judith L.

Lucas-Barbosa, Dani

Lucek, Kay

Lucena, María Isabel

Luchetti, Andrea

Luciani, Giuseppina

Luciano, Alberto Maria

Lucini, Carla

Lúcio, Marlene

Luco, Reini F.

Luculescu, Catalin Romeo

Luczak, Magdalena

Ludueña, Richard

Ludwiczuk, Agnieszka

Lugnier, Claire

Lühder, Fred

Luís, Ângelo

Lukac, Pavel
Lukas, Jan

Łukasik, Rafał

Lumniczky, Katalin

Luna, Aurelio

Lund, Jenny

Lund, Lars

Lundholm, Lovisa

Lundstrom, Kenneth

Luo, Kai Hong

Luo, Meng

Luo, Ray

Luo, Rongcong

Luo, $\mathrm{Xu}$

Luo, Zonghua

Luongo, Livio

Lupiañez, José Antonio

Lupini, Antonio

Lupo, Giuseppe

Luppi, Fabrizio

Luque, Francisco

Lusczek, Elizabeth R.

Lushchekina, Sofya

Lütjohann, Dieter

Luvisetto, Siro

Lyle, Alicia N.

Lymperopoulos, Anastasios

Lyng, Heidi

Lyu, Hailong

Lyu, Hui

Lyu, Yuanzhi

Lyubchenko, Yuri

M'Koma, Amosy E.

Ma, Guojia

Ma, Liang

Ma, Wen-Juan

Ma, Xing

$\mathrm{Ma}$, Xingmao

Ma, Zheng Feei

Maak, Steffen

Mabuchi, Mamoru

Macabeo, Allan P.

Macauley, Matthew S.

Maccallini, Cristina

Macchi, Beatrice

Macdonald, Andrew

MacDonald, Clinton C.

Macedo, Fatima

Macedo, M. Paula

Machado, Mariana V.

Machado, Ricardo

Macias, Vanessa M.

Macias-Gonzalez, Manuel

Maciejczyk, Mateusz

Maciejewska, Dominika

Macín, Stella Maris

Mack, Andreas F.

Mackereth, Cameron

MacLean, Andrew G. 
Macmillan, Colleen P.

Madala, Hanumantha Rao

Maddelein, Marie-Lise

Maddineni, Prabhavathi

MADEDDU, Roberto Beniamino

Madej, M. Gregor

Madesis, Panagiotis

Madine, Jillian

Madureira, Patrícia A.

Maeda, Akiko

Maeda, Kazuhisa

Maeda, Tomoki

Maffei, Angelo

Maga, Giovanni

Maggi, Davide

Maggini, Rita

Maggiolini, Marcello

Magherini, Francesca

Magiatis, Prokopios

Magierowski, Marcin

Magini, Alessandro

Magnaghi, Valerio

Magnaldo, Thierry

Magnani, Mauro

Magni, Fulvio

Magnusson, Karin

Magnusson, Karl-Eric

Mahalingam, Ravi

Mahavadi, Poornima

Maher, Pamela

Mahmood, Khalid

Mai, Stefania

Maida, Yoshiko

Maier, Patrick

Maiorano, Domenico

Maisch, Tim

Majeed, Waqar

Majhen, Dragomira

Majhi, Prabin

Majlessi, Laleh

Major, Ian

Majtan, Juraj

Majumdar, Soumyajit

Majumder, Mrinmoyee

Makarevich, Pavel

Makarevitch, Irina

Makena, Monish

Makieva, Sofia

Makino, Hiroshi

Makishima, Makoto

Makiyama, Takeru

Makvandi, Pooyan

Malacrinò, Antonino

Malaguarnera, Giulia

Malara, Alessandro

Malard, Veronique

Malavolta, Marco

Malde, Alpesh
Malekigorji, Maryam

Malemud, Charles

Malemud, Charles J.

Malerba, Donato

Malerba, Giovanni

Mali, Vishal

Malik, Ravinder

Mallamace, Francesco

Malle, Ernst

Mallein-gerin, Frédéric

Mallipeddi, Prema Latha

Malmberg, Per

Malpeli, Giorgio

Maltesen, Raluca

Malvolti, Maria Emilia

Mammola, Caterina Loredana

Manara, Anna

Maňásková-Postlerová, Pavla

Manavalan, Balachandran

Manca, Maria Letizia

Mancini, Cecilia

Mancini, Stéphane J. C.

Mancone, Carmine

Mancuso, Andrea

Mancuso, Cesare

Mandard, Stéphane

Mandato, Claudia

Mandava, Nanda

Mandrioli, Mauro

Manga, Prashiela

Mangalagiu, Ionel

Manini, Mhd Louai

Manjunath, Manubolu

Manley, Sharon J.

Manner, Suvi

Mannik, Jaan

Manninger, Martin

Manocha, Gunjan

Månsson, Alf

Mante, Francis K.

Manthey, John A.

Manuelli, Matteo

Manzo, Ciro

Manzoor, Rashid

Mao, Xiao Wen

Mapelli, Lisa

Marasco, Daniela

Marc, Gabriel

Marcato, Paola

Marceau, François

Marchant, Jonathan S.

Marchesan, Silvia

Marchetti, Marta

Marci, Roberto

Marco, Francisco

Marco-Contelles, José

Marcovici, Geno

Marcuzzi, Annalisa 
Marczak, Małgorzata

Marechal, Alexandre

Marechal-Drouard, Laurence

Marengo, Barbara

Margalef Jornet, Maria

Margaria, Paolo

Margaritis, Lukas

margherita, maioli

Margit, Balázs

Mari, Michele

Maria José, Rodriguez Lagunas

Maria, Naomi I.

Márialigeti, Károly

Mariconti, Mara

Mariggiò, Maria A.

Marigo, Ilaria

Marin, Daniela Eliza

Marin, José Juan García

Marincs, Ferenc

Mariniello, Loredana

Marino, Joseph

Marino, Tiziana

Mariot, Virginie

Mariotto, Sofia

Markiv, Anatoliy

Marklund, Ulrika

Markopoulou, Olga

Marla, Sandeep

Marminon, Christelle

Maroja, Luana S.

Marone, Elettra

Maroni, Paola

Maroteaux, Luc

Marqués Villavecchia, Ana M.

Marques, Natália

Marrs, James A.

Marschall, Hanns-Ulrich

Martel, Fátima

Martel, Paulo

Martí, Sergio

Martí-Calatayud, Manuel César

Martin, Abraham

Martin, Andrew V.

Martin, Chelsea K.

Martin, Finian

Martin, Francisco

Martin, Ian

Martín, Juan Francisco

Martin, Laetitia

Martin, Luc J.

Martin, Patricia

Martin, William

Martín-Antonio, Beatriz

Martín-Aragón, Sagrario

Martin-chouly, Corinne

Martinelli, Paola

Martínez De Paz, Pedro José

Martínez Iglesias, Olaia
Martínez, Alberto

Martinez, Ana

Martinez, Anne-sophie

Martínez, Antonio

Martínez, Luis Carlos

Martinez, Manuel

Martínez-Cañamero, Magdalena

Martínez-Contreras, Rebeca D.

Martínez-Esteso, María José

Martínez-Gómez, Pedro

Martinez-Lostao, Luis

Martinez-Sanchez, Aida

Martínez-Soler, Fina

Martinez-Useros, Javier

Martin-Fontecha, Alfonso

Martín-Hernández, David

Martini, Claudia

Martini, Daniela

Martini, Maurizio

Martino, Sabata

Martin-Pena, Alfonso

Martins, Albino

Martins, Ian James

Martins, Isabel

Martins, Maria Rosario

Martins, Natália

Martins, Sandra Fátima Fernandes

Martinvalet, Denis

Martín-Vasallo, Pablo

Martorana, Alessandro

Martucciello, Stefania

Maruyama, Ichiro

Maruyama, Kei

Marverti, Gaetano

Mary Ellen, Urick

Marycz, Krzysztof

Marzaro, Giovanni

Marzocco, Stefania

Marzullo, Paolo

Mas, Eric

Masaki, Tsutomu

Masalova, Olga V.

Masarik, Michal

Masauzi, Nobuo

Maserti, Bianca Elena

Mashima, Ryuichi

Masi, Antonio

Masieri, Federica

Masli, Sharmila

Masłyk, Maciej

Massarotti, Alberto

Massey, Andrew J.

Massey, Steven

Másson, Már

Massotte, Dominique

Massoumi, Ramin

Mastrangelo, Anna M.

Masuda, Junko 
Masuda, Kiyoshi

Masui, Kenta

Masullo, Mariorosario

Matarrese, Paola

Matchkov, Vladimir

Mateo, Cesar

Matesanz, Ana I.

Mateusz, Mateusz

Mathuru, Ajay S.

Mati-Baouche, Narimane

Matoba, Nobuyuki

Matrisian, Lynn M.

Matsubara, Keiichi

Matsuda, Masaru

Matsugaki, Aira

Matsugo, Seiichi

Matsui, Toru

Matsumoto, Ken'ichiro

Matsumoto, Kunio

Matsumoto, Taro

Matsumoto, Yasuhiko

Matsunami, Katsuyoshi

Matsuo, Muneaki

Matsuoka, Isao

Matsuoka, Masato

Matsushita, Takehiko

Matsuura, Bunzo

Matsuzaka, Kenichi

Matsuzaki, Goro

Matta, Csaba

Matta, Jaime

Mattei, Fabrizio

Mattijssen, Frits

Mattoscio, Domenico

Matucci Cerinic, Marco

Matusica, Dusan

Matwijczuk, Arkadiusz

Maugeri, Andrea

Maugeri, Marco

Mauk, Michael G.

Maury, Eleonore

Maurya, Shailendra

Mavian, Carla

Mavridis, Ioannis

Mavridis, Konstantinos

Mavrogonatou, Eleni

Maxwell, Anthony

Mayanil, Chandra

Mayes, Sean

Mayne, Christopher

Mayo, Kevin

Mayor, David F.

Mayorov, Alexander

Mayr, Christian

Mazars, Christian

Mazerska, Zofia

Mazor, Ohad

Mazur-Bialy, Agnieszka Irena
McArdle, Stephanie

McAuley, Julie L.

McBride, Devin W.

McCarthy, Elizabeth

McCaughan, Geoffrey William

McClelland, Sarah

McCoard, Sue

McCormick, Craig

McCubbin, Andrew

McDaneld, Tara G.

McDermott, Suzanne M.

McDonald, Allison

McDonald, Courtney

McDougal, Owen M.

McDougall, Gordon

McDowell, Andrew

McErlean, Peter

McFeeters, Robert

McGarry, Roisin C.

McGowan, Eileen

McGowan, Sheena

McGregor, Alistair

McGregor, Cecilia

McInerney, Gerald

McKay, Brian

McKee, Lauren S.

McMacken, Grace

McMillin, Matthew

McMullin, David R.

Meabon, James $S$.

Medeiros-Domingo, Argelia

Medina Piles, Vicente

Medina, Francisco Javier

Medina, Miguel

Medina, Reinhold J.

Medová, Michaela

Meesters, Dennis

Mehedi, Masfique

Mehla, Jogender

Meier, Raphäel P. H.

Meierhofer, David

Meijer, HJG

Meijer, Onno

Meijles, Daniel N.

Mejuto, Juan Carlos

Mela, Petra

Melagraki, Georgia

Melchor, Juan

Meldolesi, Jacopo

Mele, Laura

Meli, Albano

Mellody, K. T.

Melnikov, Stanislav

Melnyk, Charles

Melo, Ana M. P.

Meloni, Bruno

Meloni, Gabriele

Memarzadeh, Kaveh 
Menasché, Philippe

Menassa, Rima

Mendes, Mateus

Mendez, Juan

Méndez, Lucía

Méndez-Álvarez, Estefanía

Mendez-Sanchez, Nahun

Mendola, Diego La

Mendonca, Antonio

Mendonça, Nuno

Menendez, Esther

Meng, Dong

Meng, Hao

Meng, Ran

Meng, Weihua

Meng, Xiaoli

Meng, Yuan Xiang

Menon, Manoj

Menu, Eline

Merah, Othmane

Meraviglia, Viviana

Mercati, Francesco

Mercer, Carol

Mercier, Corinne

Mereu, Elisabetta

Merino, Jaime $\mathrm{M}$.

Merino, Ramón

Merlino, Antonello

Merlo, Giorgio

Merret, Rémy

Meruvu, Sunitha

Merzendorfer, Hans

Mesaros, Clementina A.

Messa, Piergiorgio

Messaritakis, Ippokratis

Messina, Concetta Maria

Messini, Christina I.

Messori, Luigi

Metharom, Pat

Metzinger, Laurent

Meulia, Tea

Meyer, Gregory

Meyerowitz, Elliot

Mezzasalma, Valerio

Micek, Agnieszka

Micevych, Paul

Michaelides, Sozos N.

Michalak, Izabela

Michalik, Jan

Micheau, Olivier

Micheletti, Gabriele

Michelhaugh, Sharon Kay

Micillo, Raffaella

Miele, Adriana E.

Miele, Claudia

Mielenz, Dirk

Mierke, Claudia Tanja

Miernyk, Ján A.
Mignatti, Paolo

Miguel, Silvia

Miguel, Sónia P.

Mihranyan, Albert

Mijailovich, Srba

Mikami, Koji

Mikecz, Katalin

Miki, Daisuke

Miki, Yasuhiro

Miklossy, Gabriella

Mikropoulos, Christos

Mikstacka, Renata

Milanesi, Anna

Milano, Francesco

Milano, Giuseppina

Milcovich, Gesmi

Miles, Wayne Owen

Miletta, Maria

Milhiet, Pierre-Emmanuel

Milia, Egle

Miller, Alexandra C.

Miller, Charles

Miller, Edmund

Miller, Galen

Miller, Justin

Miller, Mark

Millet, Oscar

Millhauser, Glenn

Mills, Ken

Milojevic, Tetyana

Milosavljevic, Nina

Milovanovic, Dragomir

Min, Kyung-Jin

Minamoto, Toshinari

Minarchick, Valerie

Minarovits, Janos

Minato, Ken-ichiro

Minde, David

Mine, Yuichi

Minervini, Giovanni

Minghetti, Luisa

Minkiewicz, Piotr

Minnerup, Jens

Mino, Masanobu

Minocha, Subhash C.

Minucci, Sergio

Miozzi, Laura

Miranda Salvado, Isabel Margarida

Miranda, Cristobal

Mircheff, Austin K.

Mirete, Salvador

Mirey, Gladys

Miri, Amir K.

Mirzayans, Razmik

Mirzayanz, Razmik

Misawa, Kiyoshi

Mishin, Alexander S.

Mishina, Masahiro 
Mishra, Pawan Kumar Mishra, Rupesh K.

Misra, Biswapriya Biswavas

Mitani-Ueno, Namiki

Mitchell, Cassie S.

Mitchell, John

Mitoma, Hiroki

Mitrakas, Lampros

Mitrofanis, John

Mitroulis, Ioannis

Mitsuya, Shiro

Miura, Kazuki

Miura, Naoki

Miura, Sayaka

Miyagishima, Shin-ya

Miyake, Makito

Miyamoto, Hirotaka

Miyamoto, Koji

Miyamoto, Toshinobu

Miyata, Hiroshi

Miyata, Yasuyoshi

Miyazaki, Teruo

Mizuno, Cassia S.

Mizuno, Kentaro

Mizuno, Masashi

Mizuno, Tooru

Mizzotti, Chiara

Mladěnka, Přemysl

Mlejnek, Petr

Mlera, Luwanika

Mlocicki, Daniel

Mo, Yin-Yuan

Moccia, Francesco

Mock, Beverly

Mock, Hans-Peter

Modepalli, Vengamanaidu

Modest, Dominik Paul

Modhiran, Naphak

Mody, Hardik

Moeller, Lars

Moertl, Simone

Mogi, Masaki

Mohamed, Islam

Mohamed, Salah A.

Mohammadi, Fakhrossada

Mohammadiarani, Hossein

Mohammed-Geba, Khaled

Mohan, Ganesh Babu Malli

Mohanta, Tapan

Mohd, Farooq Shaikh

Möhlendick, Birte

Mohsin Tanveer, Mohsin

Moir, Robert D.

Moisa, Sonia

Molina, Cristina E.

Molinier, Jean

Molino, Paul

Mollapour, Mehdi
Moller, Ian

Mollerup, Steen

Monaghan, James

Monastyrskaya, Katia

Moncayo, Gerald

Moncayo, Roy

Moncho Jorda, Arturo

Monetta, Tullio

Monferran, Sylvie

Mong, Mei-Chin

Mongiat, Maurizio

Moni, Lisa

Moniuszko-Szajwaj, Barbara

Monji, Akira

Montag, Judith

Montalvo-Rodríguez, Rafael

Monteiro, Alvaro

Monteiro, Susana

Montes Resano, Marta

Montesarchio, Daniela

Monticone, Silvia

Mooers, Blaine

Moon, Ilsoo

Moore, Peter

Moore, Stuart

Morales Suárez-Varela, María M.

Morales, Julio

Morales-Tirado, Vanessa

Moran, Oscar

Morandi, Luca

Moras, Dino

Morbidelli, Lucia

Moreau, Kevin

Moreau, Marc

Moreira, Paula

Morello, Silvana

Moreno, Diego A.

Moreno, J. J.

Moreno-Manzano, Victoria

Moretti, Marta

Morfini, Gerardo

Morgan, Angela

Morgese, Maria Grazia

Morgutti, Silvia

Mori, Izumi

Mori, Kazuhiro

Mori, Mattia

Moricoli, Diego

Morigi, Rita

Moriguchi, Takaya

Morikawa, Toshio

Morimoto, Masanori

Morimoto, Nobuyuki

Morimoto, Tatsuya

Morimura, Shigeru

Moriones, Enrique

Morita, Manabu

Morita, Mitsuhiro 
Morita, Naoki

Morita, Shigeto

Moriya, Takahiro

Morkunas, Iwona

Morozov, Alexander N.

Morris, Brian

Morris, Brian J.

Morrison, Melanie

Morrison, Peter

Mortara, Lorenzo

Mortiboys, Heather

Morton, Paul

Mortusewicz, Oliver

Moschovi, Maria

Mostofa, Agm G. M.

Mota, João Felipe

Mota, Manuel

Motorin, Yuri A.

Mottes, Monica

Mou, Haiwei

Moulder, Robert

Moulin, Lionel

Mourad, Raphael

Mouriño, Antonio

Mousa, Aya

Mouzeyar, Saïd

Movahedi, Ali

Mrakovcic, Maria

Mrazek, Jan

Mrówczyński, Radosław

Mucignat-Caretta, Carla

Mueller, Geoffrey

Muench, Gerald

Mühle, Christiane

Mujtaba, Shiraz

Mukai, Shoichiro

Mukaida, Naofumi

Mukherjee, Sudip

Mukohda, Masashi

Mulas, Maurizio

Mulero, Victoriano

Müller, Jens

Muller, Marc

Muller, Patricia A. J.

Müller, Rüdiger

Mullin, James M

Mullins, Robert F.

Mulloy, Barbara

Mulo, Paula

Mummadisetti, Manjula

Munaron, Luca

Munday, Michael

Muñoz Caffarel, María

Muñoz, Manuel

Muñoz-Torrero, Diego

Münscher, Adrian

Münsterberg, Andrea

Munteanu, Florentina-Daniela
Mupo, Annalisa

Muraca, Maurizio

Murakami, Yasufumi

Muraki, Katsuhiko

Murata, Jun

Murata, Takayuki

Murcha, Monika

Murdaca, Giuseppe

Murdoch, Colin

Murgia, Marta

Murotomi, Kazutoshi

Murovska, Modra

Muroya, Susumu

Murphy, Cormac

Murphy, Eain

Murray Stewart, Tracy

Muruganandan, Shanmugam

Musch, Mark

Musgrave, Ian

Mustacchi, Giorgio

Mustafa, Ghazala

Musumeci, Giuseppe

Mus-Veteau, Isabelle

Muszyński, Artur

Muthuramu, Ilayaraja

Muti, Paola

Mutsaers, Steven E.

Mutti, Luciano

Myers, Stephen

Myllykallio, Hannu

Mysiorek, Caroline

Myśliwiec, Hanna

Mystkowska, Joanna

N. Setzer, William

$\mathrm{Na}$, Dokyun

Nadia, Bazihizina

Nadia, Rucci

Nagai, Kouhei

Nagai, Noriaki

Nagai, Takayuki

Nagaraj, Chandran

Nagarajan, Sureshbabu

Nagata, Naoto

Nagata, Shinji

Nagata, Toshi

Nagatani, Naoki

Nagpal, Ravinder

Nagy, Endre

Nagy, Istvan

Nagy, Peter

Nah, Jae-Woon

Nahashon, Samuel

Naik, Surabhi

Nair, Jijeesh Ravi

Nair, Shiny

Najimi, Mustapha

Najlah, Mohammad

Nakabayashi, Kazumi 
Nakagawa, Atsushi

Nakagawa, Takuro

Nakagawa, Tsuyoshi

Nakahama, Ken-ichi

Nakajima, Masatoshi

Nakamaru-Ogiso, Eiko

Nakamura, Hitomi

Nakamura, Yoshikazu

Nakanishi, Akira

Nakano, Hiroyasu

Nakano, Toshiaki

Nakao, Reiko

Nakashima, Kazuo

Nakashima, Souichi

Nakashiro, Koh-Ichi

Nakatsuka, A.

Nakatsura, Tetsuya

Nakayama, Yuji

Nakazawa, Yasumoto

Nalam, Vamsi

Nallasamy, Palanisamy

Nalluri, Joseph

Nam, Jin-Wu

Nanavati, Charvi

Nanba, Kazutaka

Nandha Premnath, Padmavathy

Nandi, Saikat

Nanjundan, Meera

Nanni, Loris

Nano, Adela

Naoumkina, Marina

Napoli, Eleonora

Napolitano, Mariasanta

Naponelli, Valeria

Narasimhan, Madhusudhanan

Narayanan, Ajit

Narayanan, Anand

Nardelli, Carmela

Nardi, Serenella

Narkar, Vihang A.

Naruishi, Koji

Narute, Purushottam

Narváez, Manuel

Naryzhny, Stanislav

Nashima, Kenji

Nass, Norbert

Nassuth, Annette

Nastri, Flavia

Nataf, Serge

Natarajan, Mohan

Natarajan, Sathish Kumar

Natera-De Benito, Daniel

Natile, Giovanni

Nault, Rance

Naumann, Todd A.

Naumenko, Vladimir S.

Navab, Mohamad

Navarro, Estanis
Navarro, Manuel

Navazio, Lorella

Naveira, Horacio

Naviglio, Silvio

Nawaz, Muhammad

Nayak, Tapan

Naylor, Kari

Naz, Ali

Nazar, Ross N.

Nazarov, Alexey

Nazimek, Katarzyna

Nduhirabandi, Frederic

Neale, Elizabeth

Nedialkov, Paraskev Todorov

Neeland, Melanie

Nees, Matthias

Neesen, Juergen

Negishi, Takayuki

Negroiu, Gabriela

Neira, José L.

Nelson, Amanda M.

Nelson, Andrew

Nelson, Fred R. T.

Nemerow, Glen

Németh, Balázs

Nepal, Madhav

Neri, Simona

Nesmelov, Yuri E.

Nesmelova, Irina

Neuhaus, Jochen

Neumann, Carola

Neureiter, Daniel

Neutelings, Godfrey

Newburg, David

Nexø, Ebba

$\mathrm{Ng}$, Andy

$\mathrm{Ng}$, Li Fang

$\mathrm{Ng}$, Ray

Ngamcherdtrakul, Worapol

Nguyen, Cuong

Nguyen, Khue Vu

$\mathrm{Ni}$, Hongmin

Nicholls, Stephen J.

Nicholson, John W.

Nicolay, Nils

Nicoletti, Ferdinando

Nicolini, Andrea

Nicosia, Aldo

Nieberler, Markus

Niederman, Robert A.

Niehaus, Tom

Nielsen, Morten Schak

Nielsen, Vance Girard

Niemeyer, Emily D.

Niemirowicz-Laskowska, Katarzyna

Nieto, Pedro

Nieto, Teresa Pérez

Nieves-Cordones, Manuel 
Niewiadomska, Ewa

Nifli, Artemissia-Phoebe

Nigris, Filomena De

Nijhof, Inger

Nikodemova, Maria

Nikolaev, Viacheslav

Nikolakaki, Eleni

Nikolakopoulou, Angeliki M.

Niland, Stephan

Nilsen, Erik T.

Nima, Zeid A.

Nirichan, Sanoj Rejinold

Nishi, Mayumi

Nishida, Naoki

Nishigaki, Takuya

Nishiguchi, Masamichi

Nishihara, Tatsuji

Nishimura, Kaneyasu

Nishizuka, Makoto

Nisiotou, Aspasia

Nisnevitch, Marina

Nissinen, Riitta

Nistala, Ravi

Nitiss, John

Nitulescu, George Mihai

Niu, Jingwen

Nizhnikov, Anton A.

Nobili, Stefania

Noce, Annalisa

Nocentini, Giuseppe

Nociari, Marcelo

Noghero, Alessio

Noguchi, Constance Tom

Noguchi, Masayuki

Noguchi, Takuya

Nohe, Anja

Nonami, Atsushi

Nonnekens, Julie

Nonnemacher, Michael R.

Noordermeer, Daan

Norata, Danilo Giuseppe

Noren Hooten, Nicole

Norihiko, Narita

Norman, Kenneth R.

Norman, Trevor

Norris, Erin

Norton, Michael

Nosetti, Luana

Nosoudi, Nasim

Notas, George

Novellino, Ettore

Novello, Vittorino

Novick, Daniela

Novikov, Alexander

Novikova, Polina

Novio, Fernando

Novío, Silvia

Nowak, Adriana
Nowak, Felicia V.

Nowak, Renata

Nowak-Sliwinska, Patrycja

Nowicka, Grażyna

Nowicki, Janusz

Nowicki, Marcin

Nowotarski, Shannon

Nowotny, Norbert

Nozaki, Masami

Ntalli, Nikoletta G.

Ntatsi, Georgia

Ntie-Kang, Fidele

Nudler, Evgeny

Numakawa, Tadahiro

Nutt, Stephen L.

Nuytemans, Karen

Nyberg, Fred

Nylander, Karin

Nylandsted, Jesper

Nyström, Kristina

O'Brien, Edward

O'Carroll, Simon J.

Oak, Ninad

Oakhill, Jonathan S.

Obana, Akira

Obata, Hideaki

Oberg, Kerby C.

Obermeier, Birgit

Obermüller, Nicholas

Obiang-Obounou, Brice Wilfried

Ochi, Shinichiro

Ochieng, Josiah

Ochoa-Repáraz, Javier

O'Connell, Catherine

O'Connell, Richard

O'Connor, Christine

Oćwieja, Magdalena

Oda, Yukari

O'Doherty, George

Oeckinghaus, Andrea

Offord, James

Ogasawara, Toru

Ogawa, Akiko

Ogbourne, Steven

Ogden, Stacey K.

Ogi, Kazuhiro

Ogino, Shuji

Ogórek, Rafał

Ogunjirin, Adebowale

Oh, Han Bin

Oh, Jisun

Oh, Jonghyun

Oh, Man-ho

Oh, Yohan

Oh, Yoon Sin

O'Hagan, Heather M.

Ohashi, Miwa

Ohe, Kenji 
Ohkuri, Takayuki

Ohlsson, Bodil

Ohmiya, Akemi

Ohnishi, Masatoshi

Ohno, Sho

Ohshimo, Shinichiro

Ohtomo, Ryo

Ohya, Susumu

Oikawa, Akira

Oing, Christoph

Ojala, Johanna

Oka, Shuntaro

Okada, Kazunori

Okada, Muneyoshi

Okada, Taketo

Okada, Tomoyo

Okada, Yasunobu

Okajima, Hideaki

Okamoto, Masami

Okamoto, Takayuki

Okazaki, Toshiro

Okazawa, Atsushi

Okleštková, Jana

Okui, Tatsuo

Okumura, Nobuaki

Okunishi, Katsuhide

Olaru, Octavian Tudorel

Olejnik, Anna

Oleskin, Alexander V.

Oliveira, Jorge

Oliveira, Paula Alexandra Martins De

Oliveira, Raquel

Oliver, Brian

Oliver, Paula

Olmos, Dania

Olson, Michael E.

Olsson, Simon

Oltean, Mihai

Oltra, Elisa

Omais, Saad

Omar, Ahmad

Omar, Hany

Omar, Syed Haris

Omidvar, Vahid

OMORI, Yasufumi

Oniszczuk, Anna

Onorati, Maria Cristina

Onuta, Marie-Christine

Onyenwoke, Rob U

Oon, Hazel H.

Oono, Youko

Oosterwijk, Egbert

Opalińska, Magdalena

Opitz, Jörg

Oracz, Joanna

Orchard, Sandra

Orenstein, Yaron

Orio, Laura
Orlandi, Augusto

Orlov, Sergei

Orosa, Beatriz

Orren, David K.

Orsavová, Jana

Orsolic, Nada

Ortega, Eugenio Velasco

Ortega, Maria Teresa

Ortega, Natividad

Ortolano, Saida

Orton, Thomas J.

Ory, Stéphane

Orzechowski, Arkadiusz

Osanai, Tomohiro

Oshima, Yusuke

Oshiumi, Hiroyuki

Osses, Nelson

Ostaszewski, Ryszard

Oster, Henrik

Ostersetzer-Biran, Oren

Ostuni, Mariano

Oszust, Karolina

Otani, Keisuke

Othumpangat, Sreekumar

Otsuka, Yuzuru

Otsuki, Takemi

Otulak-Kozieł, Katarzyna

Otyepka, Michal

Ou, Keng-Liang

Owczarczyk-Saczonek, Agnieszka

Ozaita Mintegui, Andrés

Ozaki, Toshinori

Ozeki, Yasuhiro

Ozen, Mehmet Ozgun

Pabbidi, Reddy M

Pacak, Andrzej

Pacal, Lukas

Pacchierotti, Francesca

Pachapur, Vinayak

Pachter, Joel S.

Paci, Maurizio

Pacifici, Maurizio

Pacini, Nicola

Pacios, Luis F.

Packiam-Dayalan, Antony

Padula, Matthew

Paeschke, Nadine

Paeshuyse, Jan

Pagán, Israel

Pagani, Stefania

Pagano, Francesca

Pagano, Luca

Pagano, Stefano

Page, Melissa

Paggetti, Jérôme

Pagliai, Fernando A.

Pagliarani, Chiara

Pain, Bertrand 
Pajak, Paulina

Pakdel, Farzad

Pal Choudhuri, Shreoshi

Pal, Kasturi

Pál, Magda

Palamà, Ilaria

Palandri, Francesca

Palaniappan, Balasubramanian

Palanisamy, Arulselvan

Pałasz, Artur

Palese, Peter

Palioura, Sotiria

Palma Sircili, Marcelo

Palmblad, Magnus

Palmeira, Carlos Manuel

Palmieri, Camillo

Palmieri, Orazio

Palmirotta, Raffaele

Palomba, Letizia

Palomo Ríos, Elena

Palumbo, Carla

Palumbo, Paola

Palumbo, Vincenzo Davide

Paluszczak, Jarosław

Pan, Haihua

Pan, Hung-Chuan

Pan, Miao

Pan, Pan

Pan, Ping-Ying

Pan, Szu-Hua

Pan, Y. J.

Pan, Yangang

Panagiotidis, Mihalis

Panaro, Maria Antonietta

Panatala, Radhakrishnan

Pancsa, Rita

Panda, Amaresh C.

Pandit, Harshul

Pandolfi, Assunta

Pandolfini, Tiziana

Panfoli, Isabella

Pang, Bo

Pang, Myung-Geol

Pang, Yoonsoo

Pani, Bibhusita

Panikkanvalappil, Sajanlal R.

Pantazaki, Anastasia A.

Panwalkar, Pooja

Panzarini, Elisa

Panzella, Lucia

Paoletti, Anna Maria

Paolillo, Mayra

Paolo Busardó, Francesco

Papa, Alfredo

Papaccio, Gianpaolo

Papadimitriou, Konstantinos

Papadodima, Olga

Papamatheakis, Joseph (Sifis)
Papantonopoulos, George

Papazafiropoulou, Athanasia

Papenbrock, Jutta

Papet, Isabelle

Papini, Alessio

Papp, András

Pappas, Maria L.

Paradowska-Gorycka, Agnieszka

Parashar, Deepak

Parayath, Neha N.

Pardali, Evangelia

Pardi, Norbert

Pardo, Jose M.

Parfrey, Helen

Parikesit, Arli Aditya

Parine, Narasimha Reddy

Parisi, Ludovica

Parisini, Emilio

Park, Byung-Wook

Park, Dong Sun

Park, Enoch

Park, Hyun-Woo

Park, Jiyoung

Park, Joo-Cheol

Park, Joshua

Park, Juhyun

Park, Junsoo

Park, Ky Young

Park, Kyeongsoon

Park, Kyoung Chan

Park, Maiyon

Park, Sang Won

Park, Sung Ha

Park, Sung-Gyoo

Park, Tae Hwan

Park, Wansu

Park, Yoonseong

Parker, Dane

Parolini, Cinzia

Parrella, Edoardo

Parrizas, Marcelina

Parsons, Jason

Partanen, Jukka

Parthasarathy, Srinivas

Partsch, Hugo

Parveen, Iffat

Pascual, Alberto

Pasini, Luigi

Passamonti, Sabina

Passarinha, Luís António Paulino

Passini, Elisa

Pasternak, Taras

Pasternak, Taras P.

Pastor, Fernando

Pastores, Gregory M.

Paszek, Pawel

Patankar, Manish S.

Patel, Daxesh P. 
Patel, Deven

Patel, Hardikkumar

Patel, Yashomati M.

Paterson, David J.

Pathakoti, Kavitha

Pathania, Rajneesh

Patil, Abhinandan

Patil, Naeem K.

Patrick, Ralph

Patruno, Marco

Patterson, Eric L.

Patterson, Jennifer

Paul, Ananya

Paul, Narayan Chandra

Paulin, Roxane

Paulo, António

Pauwels, Laurens

Pavic, Valentina

Pavlik, Edward

Pavlov, Danail

Pawelkowicz, Magdalena

Pawlak, Dariusz

Pawlak, Michal

Pawłowski, Tomasz A.

Payan-Carreira, Rita

Payne, Annette

Pazdro, Robert

Pazzagli, Luigia

Pearen, Michael

Pease, James B.

Pébay, Alice

Pečenková, Tamara

Pechanova, Olga

Pécheur, Eve-Isabelle

Peck, Ammon

Pedatella, Silvana

Peddi, Prakash

Pedeux, Rémy

Pedone, Elisa

Pedrazzini, Emanuela

Pedroza, Mesias

Peet, Daniel

Peffley, Dennis M.

Pegg, Anthony E.

Pegler, Joseph L.

Pego Reigosa, Jose Maria

Peguero-Pina, José

Peguero-Pina, José Javier

Peigneur, Steve

Peiró, Sandra

Peitzsch, Claudia

Pejoski, David

Pel, Joel

Pelagalli, Alessandra

Pelin, Marco

Pellegrini, Gretel G.

Pellice, Sergio

Pellis, Alessandro
Pelosi, Paolo

Pemp, Berthold

Peña, Amado Salvador

Peña, Maria Marjorette O.

Pendyala, Gurudutt

Penfornis, Patrice

Peng, Chen

Peng, Sheng-Bin

Peng, Teng

Peng, Wen-Huang

Peng, Xinxia

Penke, Botond

Penna, Fabio

Pennock, Nathan

Pentzold, Stefan

Penuela, Silvia

Penzo, Marianna

Perche, Fédérico

Perego, Paola

Pereira, Bruno

Pereira, Carlos Dias

Pereira, Cláudia Maria F.

Pereira, Olívia R.

Perepelyuk, Maryna

Perera, Omaththage

Pérez Alvarez, Maria José

Perez Pascual, David

Perez, Juan J.

Pérez, Soledad

Perez-Clemente, Rosa Maria

Pérez-García, Selene

Pérez-Montaño, Francisco

Pérez-Pérez, José Manuel

Perez-Stable, Carlos

Peri, Francesco

Perinelli, Diego Romano

Periyasamy, Palsamy

Perl, Andras

Permyakov, Eugene

Perni, Michele

Pernisová, Markéta

Pero, Raffaela

Perrella, Giorgio

Perricone, Carlo

Perrine-Walker, Francine

Perry, Rachel

Persaud, Krishna

Perteghella, Sara

Perticone, Francesco

Perucka, Irena

Pervouchine, Dmitri

Pešić, Milica

Pessi, Gabriella

Pestov, Dimitri

Peter, Francisc

PETER, Martin G.

Peters, Eva

Peters, Verena 
Petersen, Björn

Petersen, Kristina S.

Peterson, Brandon

Peterson, Julia

Peterson, Larryn

Petit, Patrice X.

Petra I., Lorenzo

Petras, Daniel

Petrella, Antonello

Petri Jr., William A.

Petroni, Katia

Petrussa, Elisa

Petrzik, Karel

Petta, Ioanna

Pflugfelder, Stephen C.

Phang, Melinda

Phatak, Pornima

Phesse, Toby

Philipp, Stephan

Philips, Neena

Philpott, Michael

Pich, Christine

Picimbon, Jean-François

Picketts, David J.

Pickrell, Alicia

Pidatala, Venkata Ramana

Pidgeon, Graham P.

Piedade, Ana Paula

Piedras, Pedro

Piemontese, Luca

Pieraccini, Stefano

Pierangeli, Alessandra

Pierre, Sandrine

Pierrefite-Carle, Valérie

Pierzchalska, Małgorzata

Pierzchalski, Piotr

Pignatti, Patrizia

Pignochino, Ymera

Piiper, Albrecht

Pikuła, Michał

Pilarsky, Christian

Pillai, Shiv

Pillaiyar, Thanigaimalai

Pilon-Smits, Elizabeth A. H.

Pilu, Roberto

Pincus, Seth

Pingault, Lise

Pinheiro, Marina

Pinho, Brígida Ribeiro

Pinkl, Stefan

Pinto, Antonio

Pinto, Mafalda

Pinto, Milena

Pinzani, Pamela

Piplani, Honit

Piqué, Núria

Pirici, Daniel

Pirillo, Angela
Piróg, Katarzyna A.

Pirola, Luciano

Pirtoli, Luigi

Pisarcik, Martin

Pislariu, Catalina

Pissarek, Margit

Pita, Sebastian

Pitt, Samantha

Pittalà, Valeria

Pittler, Steve

Pitto, Letizia

Pitucha, Monika

Piva, Roberto

Piva, Terrence

Piya, Sarbottam

Pizzio, Gaston

Pizzolanti, Giuseppe

Placido, Jersson

Placzek, William J.

Placerer, Wojciech

Planchais, Séverine

Plano, Daniel

Plante, Isabelle

Plantier, Laurent

Plastina, Pierluigi

Platania, Chiara

Plate, Manuela

Platt, Jeffrey L.

Plaza-Zabala, Ainhoa

Płażek, Agnieszka

Pleasants, John M.

Ploner, Christian

Plonka, Przemyslaw

Płotka-Wasylka, Justyna

Plotnikov, Egor Y.

Pluquet, Olivier

Pluta, Ryszard

Pniewski, Tomasz

Pocheć, Ewa

Poggi, Alessandro

Poggio, Paolo

Pognonec, Philippe

Pogoda, Hans-Martin

Pohlmann, Stefan

Poku, Rosemary A.

Polakowski, Nicholas J.

Polanska, Kinga

Poławska, Ewa

Poli, Alessandro

Połom, Karol

Pommer, Bernhard

Ponnambalam, Sreenivasan

Ponomarev, Eugene

Pons, Antoni

Pons, Daniel Gabriel

Pons, Miquel

Pons, Sebastian

Ponz-Sarvise, Mariano 
Poon, Ivan

Poór, Miklós

Pop, Oana

Popa-Wagner, Aurel

Pope, Chad

Poplawski, Tomasz

Popoff, Steven N.

Porollo, Aleksey

Porporato, Paolo E.

Porras, Almudena

Portillo, Maria Del Carmen

Poŝa, Mihalj

Posadas, Pilar

Poschenrieder, Charlotte

Possidente, Bernard

Possik, Elite

Posta, Katalin

Potaczek, Daniel P.

Poterszman, Arnaud

Pottosin, Igor

Poudel, Barun

Pourcelly, Gerald

Pourquier, Philippe

Powell, Joann

Powell, Jonathan J.

Powrózek, Tomasz

Pozo Devoto, Victorio

Pozo, Óscar J.

Pozzobon, Michela

Prabhu, K. Sandeep

Prakasam, Sivaraman

Prakash, Aishwarya

Pratelli, Annamaria

Prats, Anne-Catherine

Pratsinis, Harris

Praud, Christophe

Prearo, Marino

Prehn, Jochen

Preissner, Klaus T.

Prentice, Howard

Pret, Anne Marie

Preto, Ana

Price, Jeffrey L.

Price, Patricia

Price, Ramona Salcedo

Prickett, Tim

Prieto, Isabel

Prieto, Manuel

Prieto-Lloret, Jesus

Primig, Michael

Primikyri, Alexandra

Primus, Carolyn M.

Prinsi, Bhakti

Proestos, Charalampos

Profumo, Elisabetta

Proietti, Primo

Prokai, Laszlo

Prokesch, Andreas
Proost, Paul

Provazník, Ivo

Prudent, Marion

Pruess, Birgit M.

Przybylska-Gornowicz, Barbara

Przybyt, Małgorzata

Psarra, Anna-Maria G.

Psomas, George

Psurski, Mateusz

$\mathrm{Pu}$, Jing

Puca, Annibale

Pucciarelli, Sandra

Puchałka, Radosław

Pucher, Johannes

Puglia, Debora

Puglia, G.

Pugliese, Giuseppe

Puigdomènech, Pere

Puiggalí, J.

Puiggalí, Jordi

Pulignani, Silvia

Punganuru, Surendra Reddy

Punyadeera, Chamindie

Puri, Akshit

Purohit, Prashant K.

Püschel, Gerhard P.

Putnam, William C. (Trey)

Pyka-Pająk, Alina

Pytka, Karolina

Qi, Robert Zhong

Qiang, Zhe

Qin, Juan

Qing, Liming

Qiu, Huan

Quarantelli, Mario

Quarck, Rozenn

Quartu, Marina

Quattrocelli, Mattia

Quesada Pérez, Víctor Manuel

Quintana, José

Raake, Philip W. J.

Rabanal Anglada, Francesc

Rabbani, Naila

Raber, Jacob

Rachek, Lyudmila

Raczkowska, Joanna

Radchenko, Eugene V.

Radic, Tanja

Radoslaw Kajetan, Kowalski

Radu, Beatrice Mihaela

Rae, Colin

Rafiei, Hossein

Raggi, Lorenzo

Ragusa, Andrea

Ragusa, Maria Antonietta

Rahat, Michal A.

Rahib, Lola

Rahimi, Farid 
Rahman Rashid, Rizwan Abdul

Rahman, Md Toufiqur

Rahman, Md. Mahbubur

Rahman, Safikur

Rahmanpour, Rahman

Rai, Krishan

Rai, Prakash

Rai, Vikrant

Raimondi, Lavinia

Raimondo, Stefania

Raina, Medha

Raina, Satish

Rais, Rana

Raj, Nitin

Rajakumar, Govindasamy

Rajala, Pauliina

Rajarathnam, Krishna

Raje, Mithun

Rajendran, Vazhaikkurichi

Rajesh Lenin, Raji

Rajić, Zrinka

Rajnarayanan, Rajendram

Rajpurohit, Surendra

Rajurkar, Mihir

Rakitin, Aleksei

Ram Kumar, Ram Mohan

Ramachandran, Anup

Ramachandran, Vinoy

Ramadori, Giuliano

Ramalingam, Nagendran

Ramalingam, Naveen

Raman, Harsh

Ramanavicius, Arunas

Ramani, Vijay

Ramaraj, Pandurangan

Ramasamy, Mohankandhasamy

Ramchandani, Divya

Ramegowda, Venkategowda

Ramich (Pivovarova), Olga

Ramil, Carlo

Ramos, Catarina

Ramsay, Michael A.

Rana, Dipak

Randall, Stephen K.

Rangachari, Manu

Rangan, Gopi

Rangel-Barajas, Claudia

Rangnekar, Abhijit

Ranieri, Girolamo

Raniszewski, Grzegorz

Ranjan, Alok

Ranjkesh, Amid

Ranzato, Elia

Rao, C. V.

Rao, Vidhya

Rapacz, Marcin

Rapak, Andrzej

Rapisarda, Venerando
Raposio, Edoardo

Räsänen, Katja

Rasche, Leo

Raschi, Emanuel

Raspanti, Mario

Rastija, Vesna

Ratajczak, Ewelina

Ratajewski, Marcin

Ratan, Aakrosh

Ratet, Pascal

Rathinasabapathy, Anandharajan

Rathinavelu, Appu

Ratkowsky, David A.

Rato, Luís Pedro Ferreira

Ratovitski, Edward

Rattan, Ramandeep

Ratz-Łyko, Anna

Rauch, Bernhard

Rauch, Jens

Rautio, Jarkko

Ravaioli, Stefano

Ravegnini, Gloria

Ravelonandro, Michel

Ravichandran, Ashwin

Ravuri, Sudheer

Rawla, Prashanth

Rawling, Tristan

Ray, William C.

Rayalam, Srujana

Razaviyan, Javad

Reader, Jocelyn C.

Reading, Benjamin J.

Redan, Benjamin

Reddy, Sakamuri

Redell, Michele

Redmer, Torben

Redondo-Muñoz, Javier

Reed, Kevin F. M.

Reese, Benjamin E.

Reeve, Vivienne

Regal, Jean

Rege, Shraddha

Rehfeld, Jens

Rehman, Junaid U.

Rehrig, Erin M.

Reinders, Joerg

Reinhold, Dirk

Reis, Henning

Reiser, Peter J.

Reisman, Scott A.

Remesh, Soumya

Ren, Dongmei

Ren, Hong

Ren, Kaixuan

Ren, Shuxin

Rena, Graham

Renaudineau, Yves

Rengaraj, Deivendran 
Renshaw, Stephen A.

Requicha, João

Resch, Ulrike

Rescifina, Antonio

Reshkin, Stephan J.

Restivo, Francesco Maria

Reszka, Edyta

Reukov, Vladimir

Reuter, Klaus

Revelli, Luca

Reverberi, Massimo

Rewatee, Gokhale

Rey, Luis

Reyes, Fernando

Reynoird, Nicolas

Rezaei-Ghaleh, Nasrollah

Reznik, Jacqueline E.

Reznik, Sandra

Rezvani, Khosrow

Rezzani, Rita

Rhee, Inmoo

Rhee, Jin-Kyu

Riar, Amanjot Kaur

Ribas, Gloria

Ribchester, Richard

Ribeiro, Clarisse

Ribeiro, João

Ribeiro, Lucas

Ribeiro-Barros, Ana I.

Ricardo, Sara

Riccardi, Carlo

Riccardo, Zenezini Chiozzi

Riccardo, Zoia

Riccetti, Laura

Ricci, Giulia

Ricciardelli, Carmela

Richa, Tambi

Richards, Lora A.

Richardson, Jonathan

Richly, Holger

Richter, Katrin

Richter, Peter

Riemann, Michael

Rienzo, Monica

Riera, Marina

Riethdorf, Sabine

Riga, Maria

Rigano, Maria Manuela

Rigas, Stamatis

Rigó, Gábor

Rigoni, Michela

Rikkerink, Erik

Riley, Kathryn R.

Rimbara, Emiko

Rimer, Mendell

Rimondini, Lia

Rinnerthaler, Mark

Riond, Joëlle
Riool, Martijn

Riquelme, Erick

Rishi, Arun

Rissiek, Bjoern

Rissiek, Björn

Riteau, Beatrice

Ritter, Christoph A.

Ritz, Ulrike

Rivas, Carmen

Rivas, Fatima

Rivera, Victor

Rivera-Vega, Loren J.

Rivero, Francisco

Rivero, Sonia

Rivero-Pérez, M. Dolores

Rivilla, Rafael

Rizos, Helen

Rizvi, Tahir

Rizzarelli, Enrico

Rizzo, Angela Maria

Rizzo, Milena

Rizzolio, Flavio

Rizzolo, Lawrence J.

Rizzuti, Bruno

Ro, Hyunju

Robert Boisivon, Helene

Robert, Christelle

Roberts, Sally

Roberts, Sigrid

Roberts, Wayne

Robertson, Avril

Robertson, Jeremy

Robin, François B.

Robin, MacDiarmid

Robinson, Clive

Robinson, David

Robinson, Karen

Robinsons, Jame

Roca, Joaquim

Rocca, Stefano

Rocha, Sonia

Rocha-Ferreira, Eridan

Rochaix, Jean-david

Rocha-Martin, Javier

Rochani, Ankit

Rocha-Rodrigues, Sílvia

Rockel, Jason S.

Rödel, Franz

Rodenbeck, Stacey Dineen

Rodolfo, Carlo

Rodrigo, Ismael

Rodrigues, Celia F.

Rodrigues, Joao

Rodrigues, Márcia T.

Rodrigues, Melanie

Rodrigues, Nuno

Rodrigues, Olivier

Rodriguez Lopez, Carlos M. 
Rodríguez, Alexander J.

Rodríguez, Amaia

Rodríguez-Alvarez, José

Rodriguez-Barbosa, José I.

Rodriguez-Cueto, Carmen

Rodriguez-Menocal, Luis

Rodriguez-Saona, Cesar

Roe, Daniel R.

Roelen, Bernard

Roffi, Alice

Rogalska, Aneta

Rogers, Melissa B.

Rogozhin, Eugene

Roh, Sangho

Rohacs, Tibor

Rohila, Jai

Rohira, Aarti

Rojo, Enrique

Rojo, Maria Angeles

Rokavec, Matjaz

Rokosz, Krzysztof

Roldão, António

Rolfo, Alessandro

Rom, Slava

Romanazzi, Gianfranco

Romani, Andrea

Romani, Massimo

Romano, Nicla

Romano-Chernac, Fabian B.

Romei, Cristina

Romeo, Giovanna

Romeo, Roberto

Romero, Alejandro

Romero, Fernando M.

Romero, Marta R.

Romero-Canelón, Isolda

Romero-García, Juan M.

Rom-Jurek, Eva-Maria

Rommer, Paulus

Romo, Maria Del Mar Ortega-villaizan

Ron, Eliora

Ronan, Kapetanovic

Ronca, Alfredo

Rondanino, Christine

Ronellenfitsch, Michael W.

Ronkainen, Justiina

Rönö, Kristiina

Rooj, Arun

Root-Bernstein, Robert

Ropka-Molik, Katarzyna

Roques, Christian-François

Rosa, Vinicius

Rosado, Juan A.

Rosato, Antonio

Rosellini, Daniele

Rosenbluth, Jennifer M.

Rosencrantz, Ruben R.

Rosenthal, Ken
Roseti, Livia

Rosicka-Kaczmarek, Justyna

Rosołowska-Huszcz, Danuta

Ross, Ian

Ross, Ian L.

Rossa Jr, Carlos

Ros-Santaella, José Luis

Rossignol, Julien

Rostaing, Lionel

Rosti, Vittorio

Roterman, Irena

Roth, William

Rothman, Alexander M.K.

Rotili, Dante

Rotllant, Josep

Rouached, Hatem

Roubelakis-Angelakis, Kalliopi

Rouch, Al

Rouhiainen, Ari

Rounbehler, Robert J.

Rouse, Matthew N.

Rousseau, Anne Sophie

Rousset, Raphaël

Roussis, Vassilios

Rout, Bhimsen

Routledge, Michael

Rovero, Paolo

Roy Choudhury, Gourav

Roy Choudhury, Swarup

Roy, Anuradha

Roy, Dipankar

Roy, Kasturi

Roy, Sonali

Roy, Sourav

Roychoudhury, Aryadeep

Royo, Felix

Rozhon, Wilfried

Różycki, Bartosz

Rua, Diego

Rübe, Claudia

Rubio Valverde, Lourdes

Rubio, Vicente

Rubis, Blazej

Rucker, Robert B.

Ruckert, Felix

Ruddraraju, Kasi Viswanatharaju

Ruden, Douglas

Rudolph, Bryan

Rudrabhatla, Sairam

Rudykh, Stephan

Rudzitis-Auth, Jeannette

Ruelland, Eric

Ruggeri, Francesco

Ruggeri, Paolo

Ruggerone, Paolo

Rughoobur, Girish

Ruiu, Luca

Ruiz Lozano, Juan 
Ruiz, Mario

Ruiz-Echevarria, Maria J.

Ruiz-Ederra, Javier

Ruiz-Pérez, María Victoria

Rund, Deborah

Ruoppolo, Margherita

Rupasinghe, Thusitha

Rupenthal, Ilva

Rupérez, Azahara Iris

Rupprecht, Rainer

Rurek, Michal

Rusciano, Dario

Ruscica, Massimiliano

Rusmini, Paola

Russ, Brendan

Russo, Annapina

Russo, Antonella

Russo, Antonio

Russo, Giulia

Russo, Maria

Russo, Nino

Ruthstein, Sharon

Ruzicka, Jiri

Ruzicka, Kamil

Ryabchikova, Elena I.

Ryan, Michael P.

Ryan, Robert

Rybak, Leonard

Rytel, Liliana

Ryu, Ji-Kan

Ryu, Jong

Rzepecki, Ryszard

Rzymowska, Jolanta

S. Douches, David

Saad, Hosam

Sabater, Bartolome

Sabirov, Ravshan Z.

Saccà, Barbara

Sacchi, Gian Attilio

Saccone, Valentina

Sachkova, Maria

Sacitharan, Pradeep Kumar

Sadeghi, Sheila

Sadler, Anthony

Saez, Carmen

Safavi-Hemami, Helena

Saferding, Victoria

Sagnou, Marina

Sagui, Celeste

Saha, Achinto

Saha, Shyamali

Saha, Surya

Sahab, Sareena

Sahara, Naruhiko

Sahoo, Pabitra

Sahoo, Pabitra Kumar

Sahu, Jagajjit

Sai, Kiran Kumar Solingapuram
Saielli, Giacomo

Saini, Shikha

Sainlos, Matthieu

Saisho, Yoshifumi

Saito, Mariko

Saito, Yoshiro

Saito, Yukihiro

Saitoh, Kenji

Saitoh, Masao

Saitoh, Yasukazu

Saiz-Fernández, Iñigo

Sakaguchi, Masakiyo

Sakaguchi, Yusuke

Sakai, Atsushi

Sakai, Ryuichi

Sakakibara, Iori

Sakamoto, Kensaku

Sakamuro, Daitoku

Saki, Najmaldin

Sakkas, Lazaros I.

Sakr, Soulaiman

Sakuma, Kunihiro

Sala, Roberto

Salaheen, Serajus

Salas, Carlos

Saleem, Ayesha

Saleem, Muhammad

Salehin, Mohammad

Saler, Marco

Salgar, Shashikumar

Salifoglou, Athanasios

Salim, Vonny

Salminen, Juha-Pekka

Salt, Ian

Salta, Evgenia

Saluk-Bijak, Joanna

Salvarani, Nicolo'

Salvarani, Nicolò

Salvatore, Giuliana

Salvatore, Pepe

Samaja, Michele

Sambuy, Yula

Šamec, Dunja

Samhan-Arias, Alejandro

Sammi, Shreesh

Sampathi, Shilpa

Samuels, Ivy

Samuha, Shmuel

Samways, Damien S. K.

San Fabian, Emilio

San Jose, Michael

Sanches Silva, Ana

Sánchez Pina, Amelia

Sánchez-Fidalgo, Susana

Sanchez-Martinez, Melchor

Sánchez-Purrà, Maria

Sancho, Ana

Sanders, Matthew 
Sandow, Shaun L.

Sanejouand, Yves-Henri

Sanghera, Paul

Sangireddy, Sasikiran Reddy

Sani, Marc-Antoine

Sanjust, Enrico

Sankaran, Ganapathy Subramanian

Sankaranarayanan, Nehru Viji

Sanmartín Grijalba, Carmen

Sanner, Michel F.

Sano, Naoto

Sansone, Clementina

Santacroce, Luigi

Santamaria, Rita

Santarelli, Andrea

Santarelli, Lory

Santhakumar, Abishek

Santin, Matteo

Santini, Emanuela

Santini, Valeria

Santino, Angelo

Santofimia-Castaño, Patricia

Santolla, Maria Francesca

Santoni-Rugiu, Eric

Santos, Ana Paula

Santos, Inês

Santos-Gallego, Carlos

Santos-Ruiz, Leonor

Santucci, Annalisa

Santulli, Gaetano

Sanz Garcia, Andres

Sanz-Clemente, Antonio

Sanz-Herrera, Jose A.

Saotome, Masao

Saponaro, Chiara

Sapudom, Jiranuwat

Saraiva, Nuno

Sarapultsev, Alexey

Sarbini, Shahrul

Saretzki, Gabriele

Sargueil, Bruno

Sarkar, Chandrani

Sarkar, Dhruba

Sarkar, Sibaji

Sarkes, Deborah A.

Sarnowski, Tomasz

Sarti, Alba Clara

Sasaki, Hideo

Sasaki, Jun-Ichi

Sasaki, Kazuhiro

Sasaki, Masanori

Sasaki, Motoko

Sasaki, Reina

Sase, Ajinkya

Sassi, Yassine

Satake, Hironaga

Satani, Nikunj

Satbhai, Santosh
Satbhai, Santosh Balasaheb

Sathe, Gajanan Jalindarnath

Sato, Fuyuki

Sato, Hiromi

Sato, Kenji

Sato, Koji

Sato, Shin

Sato, Takuichi

Sato-Bigbee, Carmen

Satoh, Shigeru

Satoh, Takaya

Satoh-Nagasawa, Namiko

Satoshi, Kameshima

Satou, Ryousuke

Sattar, Sampurna

Sau, Samaresh

Saunders, Nicholas A.

Saurav, Kumar

Sauter, Daniel

Savaraj, Niramol

Savary, Stéphane

Savenkov, Eugene

Savi, Monia

Savoie, Jean-Michel

Savu, Diana

Saw, Constance L. L.

Sawada, Hiroshi

Sawada, Shojiro

Sawai, Hirofumi

Sawicka-Powierza, Jolanta

Sawicki, Rafal

Scala, Valeria

Scalise, Mariafrancesca

Scandurra, Roberto

Scarafoni, Alessio

Scarel-Caminaga, R. M.

Schaap, Frank

Schaberle, Till F.

Schäfer, Wilhelm

Schaffer, Arthur A.

Schäffner, Anton

Schaiquevich, Paula

Scheicher, Ralph H.

Schéle, Erik

Schenk, Michael

Schepisi, Giuseppe

Scherer, Günther

Schiavone, Marco

Schiffner, Rene

Schijns, Virgil E. J. C.

Schild-Poulter, Caroline

Schillaci, Domenico

Schininà, Maria Eugenia

Schinke, Thorsten

Schipper, Hyman M.

Schirmer, Bastian

Schlachetzki, Johannes

Schlaepfer, Isabel R. 
Schlessinger, Avner

Schlueter, Klaus-Dieter

Schmidt, Marc

Schmidt, Rebecca

Schmitt-Egenolf, Marcus

Schmitz, Boris

Schmitz, Hans-Peter

Schmoelzl, Sabine

Schmoll, Dieter

Schmutz, Marc

Schneider, Anja

Schneider, Barbara

Schnichels, Sven

Schoenhagen, Paul

Schomacher, Lars

Schönberger, Stefan

Schönitzer, Veronika

Schönthal, Axel H.

Schouten, Alexander

Schrock, Morgan

Schroeder, Christina

Schroeder, Hilke

Schubert, Mario

Schuetz, Alexandra

Schuhmann, Michael K.

Schulte, Carsten

Schulz, Florian

Schulz, Margot

Schulz, Wolfgang A.

Schulze, Gundula

Schulze, Johannes

Schulz-Heddergott, Ramona

Schumacher, Fabian

Schutte, Stacey

Schwaminger, Sebastian

Schwan, William

Schwarz, Steven M.

Schweizer, Michael

Schwelm, Arne

Schwen, Daniel

Schwerdt, Julian

Schwertfeger, Kaylee

Schwiebs, Anja

Sciacchitano, Salvatore

Scicchitano, Pietro

Sciortino, Maria Teresa

Scorziello, Antonella

Scott, Daniel

Scotto D'Abusco, Anna

Scribante, Andrea

Scrima, Mario

Sebastian, Aimy

Sebastian, Carlos

Sebastiana, Mónica

Sebastiani, Federico

Sebio, Ángel Abuelo

Seca, Ana Maria Loureiro Da

Sechi, GianPietro
Seckinger, Anja

Sedic, Mirela

Sedio, Brian E.

Sediva, Anna

Sedlačík, Michal

Seetharam, Arun

Segura, Ana

Segura, Joan

Segura, Juan

Seibert, Franz Josef

Seidel, George E.

Seifert, Georg J.

Seiler, Magdalene J.

Seki, Yoshinobu

Sękowski, Szymon

Selby, Katja

Seliger, Corinna

Seligmann, Hervè

Selim, Sameh

Sellayah, Dyan

Sellix, Michael T.

Semenyuk, Pavel

Sempere, Lorenzo F.

Sena, Armando

Senatov, Fedor S.

Senbonmatsu, Takaaki

Sendón, Raquel

Sengupta, Arjun

Sengupta, Debrup

Senior, Alistair M.

Seo, Hak Soo

Seoane-Collazo, Patricia

Sepodes, B.

Serafini, Gianluca

Serefko, Anna

Sergeant, Kjell

Sergi, Consolato

Serio, Gabriella

Serralheiro, Pedro

Serrano, Irene

Servis, Michael

Sesaki, Hiromi

Sessoms, Florence J.

Sesti, Federico

Sestili, Francesco

Seth, Devanshi

Seth, Ratanesh

Sethi, Gautam

Sethmann, Ingo

Setzer, William N.

Severson, Aaron F.

Seystahl, Katharina

Sgarzi, Massimo

Sgrignani, Jacopo

Shah, Ajit

Shah, Dilip

Shah, Manish

Shah, Ruchi 
Shahid, Muhammud Qasim

Shanjani, Yaser

Shanmugam, Avinash

Shapiro, Bruce A.

Sharan, Riti

Sharif, Ariane

Sharma, Amrish

Sharma, Arishya

Sharma, Arun

Sharma, Atul

Sharma, Bhesh

Sharma, Deepika

Sharma, Neeru

Sharma, Rohit

Sharma, Shweta

Sharma, Sudha

Sharma, Sunny

Sharman, Edward H.

Shavandi, Amin

Shaw, Lisa

Shcherbik, Natalia V.

Shearrer, Grace E.

Sheen, Shyn-Shin

Shehata, Nader

Shehu, Amarda

Sheldrake, Helen

Shelke, Ganesh

Shen, Jia Zack

Shen, Shengrong

Shen, Weiran

Shen, $Y i$

Shen, Yi-Ping

Shen, Yu-Fang

Sheng, Ruilong

Sherbenou, Daniel W.

Sheri, Madhu

Sherr, David H.

Sheth, Sandeep

Sheu, Ming-jyh

Shi, Haifei

Shi, Hua

Shi, Jian

Shi, Jiaqi

Shi, Zhi

Shiao, Young-Ji

Shibuya, Hiroshi

Shichita, Takashi

Shiga, Takashi

Shigeishi, Hideo

Shigeru, Hishinuma

Shigeru, Yatoh

Shih, Yu-Ru

Shiina, Takashi

Shikama, Yosuke

Shim, Hyunbo

Shim, Joon W.

Shim, Rosalyn

Shim, Yhong-hee
Shimada, Masako

Shimada, Tsutomu

Shimada, Yasuhito

Shimizu, Takahiko

Shimizu, Takatsune

Shimo, Tsuyoshi

Shimodaira, Shigetaka

Shimura, Hanako

Shinde, Suhas

Shingu, Takashi

Shioda, Norifumi

Shiomi, Daisuke

Shiozawa, Yusuke

Shipley, Paul

Shirahata, Tatsuya

Shiraishi, Takehiko

Shirasuna, Koumei

Shirato, Hiroki

Shivanna, Binoy

Shojaei, Shahla

Shortt, Jake

Shosha, Esraa

Shostak, Anton

Showalter, Allan

Shu, Jiang

Shu, Longfei

Shukla, Hem

Shults, Elvira E.

Shunmugam, Arun

Siatka, Tomáš

Sicinski, Rafal

Siddappa, Byrareddy

Sidhu, Rohini

Sidorenko, Viktoriya S.

Sidransky, Ellen

Sié, Pierre

Sieber, Fritz

Siegel, Sue Rutherford

Siemer, Ansgar B.

Siemianowski, Oskar

Sier, Cornelis F.M.

Sierecki, Emma

Signore, Giovanni

Sigusch, Bernd W.

Sikavitsas, Vassilios

Sil, Payel

Šiler, Branislav

Sillence, Dan

Silman, Israel

Silva, Adriana Ribeiro

Silva, Ana M.

Silva, Luís

Silva, Margarida F. B.

Silva, Nuno A.

Silvagno, Francesca

Silvan, Unai

Silvente-Poirot, Sandrine

Silvestre, Samuel 
Silvestri, Ida

Sim, Sheina B

Simakov, S. S.

Simard, Alain

Simard, Marc

Simecka, Jerry W.

Šimić, Goran

Simitzi, Chara

Simitzis, Panayiotis

Šimko, Fedor

Simm, Stefan

Simmen, Rosalia C. M.

Simo, Ladislav

Simões, Marta Filipa

Simon, Istvan

Simone, Angela De

Simonescu, Claudia Maria

Simoni, Jan

Simón-Mateo, Carmen

Simonsen, Henrik Toft

Simon-Yarza, Teresa

Simopoulou, Mara

Simpson, David A. C.

Simpson, Julie E.

Sims-Robinson, Catrina

Simurda, Tomas

Singh, Abhishek

Singh, Amareshwar

Singh, Anil

Singh, Anurag

Singh, Arunima

Singh, Ashok K.

Singh, Brijesh Kumar

Singh, Chandra

Singh, Chanpreet

Singh, Harminder Pal

Singh, Jugpreet

Singh, Kamalendra

Singh, Khushwant

Singh, Kumar Saurabh

Singh, Narinder

Singh, Naveen Kumar

Singh, Raksha

Singh, Shakti

Singh, Sonal

Singh, Vishal

Singh-Cundy, Anu

Singla, Bhupesh

Sinha, Amit

Sinha, Kalyan

Siniscalco, Dario

Sinyukov, Alexander

Sionov, Edward

Sips, Patrick

Sireci, Guido

Siristatidis, Charalampos

Sirko, Swetlana

Sirotkin, Alexander V.
Sirtori, Cesare

Şişecioğlu, Melda

Sita, Giulia

Sitarek, Przemysław

Sitcheran, Raquel

Sitz, Rachael A.

Sivakumar, Sushama

Sivaprakasam, Sathish

Sivasubramaniyan, Kavitha

Siwek, Agata

Sixou, Sophie

Sizochenko, Natalia

Sizun, Christina

Sjöberg, Folke

Skaaby, Tea

Skalsky, Rebecca

Skendros, Panagiotis

Skiba, Mohamed

Skirtach, Andre

Skoneczny, Marek

Skopouli, Fotini Nikolaos

Skorik, Yury A.

Skórzyńska-Dziduszko, Katarzyna E.

Skowronska, Agnieszka

Skriver, Karen

Skurla, Carolyn

Slamova, Kristyna

Slater, Clarke

Slawson, Chad

Śliwka, Jadwiga

Sloan, Kenneth B.

Slominski, Andrzej

Slootweg, Erik J.

Smagghe, Guy

Small, Leo J.

Smani, Younes

Smertenko, Andrei

Smiesko, Martin

Śmieszek, Agnieszka

Smit, Linda

Smith Callahan, Laura A.

Smith, Brittany L.

Smith, Hugh A.

Smith, Jessica

Smith, Jill P.

Smith, Kimberly

Smith, Matthew

Smith, Micholas Dean

Smith, Patrick J.

Smith, Stephanie J.

Smolikova, Galina

Smyth, James

Smyth, Redmond

Snegur, Lubov

Snowden, Timothy

Soares, Claudio M.

Soares, Paula

Sobeh, Mansour 
Sobotta, Lukasz

Soe, Kent

Soenen, Stefaan J.

Sofronov, Georgy

Sohl, Christal

Sokolove, Jeremy

Solano, Francisco

Soler, Miguel Ángel

Solomon, Melani

Solovyev, Andrey

Sommariva, Michele

Sommerhalter, Monika

Son, Deok-soo

Son, Hokyoung

Sonda, Sabrina

Song, Chunhua

Song, Guoqing

Song, Jeongmin

Song, Jia L.

Song, Jiancheng

Song, Lu-Jie

Song, Shijie

Song, Su

Song, Sung Ho

Soni, Dheeraj

Sonkoly, Enikö

Sonntag, Kai

Soole, Kathleen

Soós, Vilmos

Sophia, Chernikova

Sorg, Olivier

Soriano, Jose Miguel

Sorrell, J. Michael

Sorrenti, Valeria

Sorrentino, Elena

Sosna, Justyna

Soteriou, Georgios

Sottnik, Joseph

Souček, P.

Soucy, Shannon

Soudeyns, Hugo

Soulika, Athena

Sounni, Nor Eddine

Sousa, Sandra

Sousounis, Konstantinos

South, Andrew

Souza-Fonseca-Guimarães, Fernando

Soverini, Simona

Sowa, Ireneusz

Sowndhararajan, Kandhasamy

Spagnuolo, Rocco

Španič, Valentina

Spassov, Sashko

Spegazzini, Nicolas

Spégel, Peter

Spencer, Charles T.

Spencer, Thomas

Spengler, Dietmar
Speranza, Giovanna

Spiekerkoetter, Edda

Spinazze, Andrea

Spingler, Bernhard

Spizzo, Riccardo

Spring, Kevin

Spyrakis, Francesca

Squire, John

Šrámek, Jan

Sreedasyam, Avinash

Sridhar, Jayalakshmi

Sripathy, Smitha

Sriram, Gopu

Srivastava, Akhil

Srivastava, Atul

Srivastava, Saurabh

Srivatsan, Malathi

Srivenugopal, Kalkunte

Sroka, Zbigniew

St John, James A.

St. Hilaire, Cynthia

Stacchiotti, Alessandra

Stachowska, Ewa

Stafford, James

Stahl, Felix

Stahl, Frank

Staines, Katherine

Stakenborg, Nathalie

Stam, Remco

Stańczyk, Wlodzimierz A.

Stanek, Agata

Stange, Katja

Stanley, Jone A.

Starodubova, Elizaveta

Starowicz, Małgorzata

Starr, Amanda

Stasiak, Andrzej

Stasko, Jan

Stathopulos, Peter

Staunstrup, Nicklas Heine

Stecca, Barbara

Steen, Ida

Steenbergen, Renske

Stefani, Ambra

Stefani, Laura

Stefanovic, Branko

Steffan, Joshua J.

Steger, Gertrud

Steinhoff, U.

Steinle, Jena

Steinmeyer, Juergen

Stenbeck, Gudrun

Stendahl, Olle

Stephan, Holger

Stepien, Ewa

Stępień, Łukasz

Stepnik, Maciej

Stevens, Joanne M. 
Stevenson, Bradley

Stewart, Beverly

Stewart, Delisha

Stewart, Jason

Stewart, Michael W.

Stieber, Juliane

Stieger, Bruno

Stilli, Donatella

Stine, Keith J.

Stjohn, Justin

Stobdan, Tsering

Stobiecka, Magdalena

Stobiecki, Maciej

Stocco, Carlos

Stojko, Jerzy

Stoleru, Elena

Stolfi, Carmine

Stopeck, Alison T.

Stopka, Tomáš

Štorchová, Helena

Storkus, Walter

Storlazzi, Aurora

Storti, Paola

Stossi, Fabio

Stournaras, Christos

Stout, David A.

Stout, Michael

Stout, Randy

Stow, Jenny

Stoychev, Georgi

Štrac, Dubravka Švob

Strasser, Richard

Stratmann, Bernd

Stratmann, Johannes

Straus, Suzana K.

Strauss, Laura

Strauss, Olaf

Strauss, Phyllis

Strauss, Sarah

Strekalova, Tatyana

Streubel, Jana

Striker, Rob

Stroffekova, Katarina

Strosznajder, Joanna B.

Strube, Jochen

Strudwick, Xanthe

Struga, Marta

Stubbs, Brianna

Studzińska-Sroka, Elżbieta

Studzinski, George P.

Stührwohldt, Nils

Stupka, Nicole

Sturlese, Mattia

Stylianou, Andreas

$\mathrm{Su}$, Meng

Subhi, Yousif

Subramani, Mayavan

Subramaniam, Prasad
Subramanian, Veedamali

Sud'ina, Galina F.

Suda, Kenichi

Sudheeran, Pradeep Kumar

Sudhir, Putty Reddy

Sudhir, Putty-Reddy

Sugase, Kenji

Sugawara, Akihiro

Sugimoto, Naotoshi

Sugimura, Haruhiko

Sugiura, Daisuke

Sugiura, Yuki

Suh, Dae-yeon

Suh, Won Hyuk

Suhail, Yasir

Suidan, Georgette

Suk, Ki Tae

Sukhov, Vladimir

Sukocheva, Olga

Sukumaran, Sunitha

Sulatskaya, Anna

Sulek, Karolina

Suliburska, Joanna

Sullivan, Chris

Sullivan, Katie E. O'

Sullivan, Lucas

Sultan, Faraz

Suman, Shubhankar

Sun, Changpo

Sun, Chengwen

Sun, Conroy

Sun, Fengjie

Sun, Jie

Sun, Jingjing

Sun, Jun

Sun, Lu

Sun, Mingkuan

Sun, Qian

Sun, Qing

Sun, Wei

Sun, Weijing

Sun, Wenyue

Sun, Wujin

Sun, Xiaoming

Sun, Xinghui

Sun, Yingjie

Sunami, Yoshiaki

Sundar, Reshma

Sundaram, Uma

Sundaramoorthy, Pasupathi

Sung, Sibum

Sunna, Anwar

Sunohara, Yukari

Sunseri, Francesco

Suntharalingam, Kogularamanan

Supernat, Anna

Surapathrudu, Kanakala

Suravajhala, Prashanth 
Surdacki, Andrzej

Surguchov, Andrei

Suryawanshi, Vasantika

Susic, Michael

Sutaria, Dhruvitkumar

Sutherland, John B.

Suzuki, Hideo

Suzuki, Hiroetsu

Suzuki, Hiroshi I.

Suzuki, Katsuhiko

Suzuki, Takafumi

Suzuki, Yuki

Svedas, Vytas

Svob Strac, Dubravka

Swamy, Ganesh

Swanberg, Maria

Swarbreck, Stéphanie M.

Swayne, Leigh-Anne

Swerdlow, Russell H.

Swevers, Luc

Swiatkowska-Warkocka, Zaneta

Swierczynski, Julian

Syed, Viqar

Sykes, Pamela

Sykora, Peter

Symkal, Petr

Symonova, Radka

Szabados, László

Szablewski, Leszek

Szafranski, Krzysztof

Szalay, Aladar A.

Szaleniec, Maciej

Szatmári, Tünde

Szczepanski, Caroline

Szczolko, Wojciech

Szebeni, Gábor J.

Szeiffova Bacova, Barbara

Szeliga, Monika

Széll, Márta

Szewczuk, Myron R.

Szewczyk, Katarzyna

Szewczyk, Nathaniel J.

Szklarz, Grazyna

Szopa, Aleksandra

Sztanke, Małgorzata

Sztuba-Solinska, Joanna

Szweda, Piotr

Szymanowska, Urszula

Szymańska, Renata

Tabata, Yasuhiko

Tabish, Tanveer

Tacconelli, Stefania

Tadashi, Watabe

Taddei, Maria Letizia

Tadepalli, Sirimuvva

Tadesse, Wuletaw

Tadych, Mariusz

Tafuri, Agostino
Taghibiglou, Changiz

Taglietti, Angelo Maria

Taguchi, Ayumu

Taguchi, Kumiko

Taguchi, Y-h.

Taguchi, Yoichiro

Tahimic, Candice

Tai, TC

Tai, Wanbo

Taira, Junsei

Tajima, Tsuyoshi

Tajiri, Naoki

Takabe, Wakako

Takahara, Terunao

Takahashi, Akihisa

Takahashi, Hiroki

Takahashi, Ken

Takahashi, Nobuyuki

Takahashi, Ohgi

Takahashi, Shinya

Takahashi, Taku

Takahashi, Toshio

Takaki, Akinobu

Takaki, Manabu

Takakura, Nobuyuki

Takamatsu, Daisuke

Takamatsu, Shigeyuki

Takano, Katsura

Takasawa, Shin

Takaya, Junji

Takayama, Kenichi

Takayama, Yoshiharu

Takebayashi, Shin-ichiro

Takegahara, Noriko

Takeishi, Yasuchika

Takemori, Hiroshi

Takemoto, Hiroyasu

Takeuchi, Takeshi

Takeuchi, Tomoharu

Takó, Miklós

Takumi, Shigeo

Taleb, Nadine

Tallerico, Rossana

Talukder, Poulami

Tamaki, Tetsuro

Tamama, Kenichi

Tamamis, Phanourios

Tamarat, Radia

Tamarit, Jordi

Tamma, Grazia

Tammimies, Kristiina

Tamura, Yukinori

Tan, C. Sabrina

Tan, Dun-Xian

Tan, Ek Han

Tan, Joanne

Tan, Shyh-Han

Tanabe, Katsuyuki 
Tanabe, Kenji

Tanaka, Hiromitsu

Tanaka, Masato

Tanaka, Nobuyuki

Tanaka, Shigeo

Tanaka, Shinji

Tanaka, Takuji

Tanaka, Toshiaki

Tanaka, Yasuo

Tanaka, Yoshiaki

Tanaka, Yoshio

Tang, Liping

Tang, Ming-Jer

Tang, Renjie

Tang, Youhong

Tang, Yuan

Tani, Akio

Taniguchi, Hiroaki

Taniguchi, Kohei

Tanimizu, Naoki

Tankiewicz-Kwedlo, Anna

Tanner, Eden

Tanner, Nathan

Tanoi, Keitaro

Tanonaka, Kouichi

Taoka, Yousuke

Taoufik, Era

Tappia, Paramjit S.

Taran, Elena

Tarantini, Stefano

Tarantino, Giovanni

Taraschi, Susanna Maria

Tarasova, Nadya I.

Tariq, Mohammad

Tarozzi, Andrea

Tarro, Giulio

Tashima, Toshihiko

Tashiro, Hirotaka

Tata, Ada Maria

Tate, Shin-ichi

Tateishi, Kensuke

Tathireddy, Prashant

Tatsuke, Tsuneyuki

Tatullo, marco

Taub, Mary

Taube, Joe

Taura, Kojiro

Tavanti, Francesco

Tavares, Anthony

Tavío, María Del Mar

Tavladoraki, Paraskevi

Tavori, Hagai

Tawfik, Sherif Abdulkader

Taylor, Erika A.

Taylor, Kathryn

Tchorbanov, Andrey

Te Riele, Hein

Te Velthuis, Aartjan
Tebani, Abdellah

Tedeschi, Rosemarie

Tedesco, Idolo

Tedesco, Serena

Tee, Andrew R.

Teeri, Teemu

Teige, Markus

Teixeira Tomaz, Cândida

Teixeira, Ana Luísa

Tejada, Silvia

Telser, Joshua

Temel, Aslihan

Temeyer, Kevin B.

Tempera, Gianna

Tempera, Italo

Tempfer, Herbert

Tencerova, Michaela

Tenhaken, Raimund

Tennessen, Jason

Teraishi, Masayoshi

TERAMOTO, Hidetoshi

Teramoto, Yoshikuni

Terpitz, Ulrich

Terracciano, Daniela

Terreri, Sara

Terriente, Javier

Terry, Randall G.

Terzaghi, William

Terzaghi, William Bryan

Terzi, Valeria

Teschendorff, Andrew E.

Teschke, Rolf

Tessarz, Peter

Testa, Ugo

Testai, Fernando D.

Teter, Ken

Tetreau, Guillaume

Tetz, George

Tevosian, Sergei G.

Teymourian, Hazhir

Tezil, Tugsan

Thackray, Alana

Thakor, Nehal

Thakurela, Sudhir

Thaler, Roman

Thalhammer, Gregor

Thammina, Chandra

Thangaraj, Annadurai

Theile, Dirk

Thelin, Eric

Thiel, Teresa

Thijs, Sofie

Thilmony, Roger

Thiriet, Marc

Thomas, Peter

Thomas, T. J.

Thomas, Wayne

Thomine, Sébastien 
Thompson, Jeffrey

Thompson, Karl Manley

Thompson, Kim

Thomson, Jennifer

Thorn, Peter

Thornburg, Natalie

Thornton-Kurth, Kara

Thorpe, Chavaunne

Thrasyvoulou, Andreas

Thrivikraman, Greeshma

Thummel, Ryan

Thyparambil, Aby

Tian, Bin

Tian, Jiang

Tian, Li

Tian, Xiaoyu

Tian, Zhongmin

Tiano, Luca

Ticozzi, Nicola

Tiera, Marcio

Tietjen, Ian

Tiffen, Jessamy C.

Tigyi, Gábor J.

Tikhonov, Denis B.

Tikhonov, Vladimir

Tilbrook, Alan

Tilley, Michael

Timani, Khalid

Timofeev, Vladimir I.

Timoshenko, Alexander V.

Tiriveedhi, Venkataswarup

Tischler, Dirk

Tiwari, Pooja

Tiwari, Rakesh K.

Tiwari, Vijay

Toca-Herrera, Jose

Tocchetti, Carlo Gabriele

Toden, Shusuke

Todi, Sokol

Toh, Ban-Hock

Toh, Wei Seong

Toietta, Gabriele

Toillon, Robert-Alain

Toiu, Anca

Tokas, Theodoros

Toldrá, Fidel

Tolkatchev, Dmitri

Tolosa, Ezequiel

Tomar, Dhanendra

Tomasetti, Carmine

Tomasetti, Marco

Tomassetti, Antonella

Tomasson, Michael

Tomaszewska, Ewa

Tomatsu, Shunji

Tombesi, Sergio

Tomczykowa, Monika

Tominaga, Motoki
Tommasini, Steven M.

Tomoaki, Sakamoto

Tomuleasa, Ciprian

Tong, Qiang

Toniolo, Antonio Q.

Toorie, Anika

Tooze, Reuben

Top, Deniz

Torigoe, Kanjiro

Tornesello, Maria

Torras, Joan

Torre, Maria Luisa

Torrente, Angelo

Torres, Adrian Gabriel

Torres, Magdalena

Torres-Reveron, Annelyn

Torrieri, Elena

Tortora, Paolo

Torzewski, Michael

Tósaki, Árpád

Tosato, Giovanna

Tosh, Susan

Totah, Rheem

Toulany, Mahmoud

Touma, Chadi

Tovoli, Francesco

Trabace, Luigia

Trabucchi, Michele

Traini, Tonino

Tran, Fabien

Tran, Kim

Tranulis, Michael

Travaglione, Sara

Travers, Jeffrey Bryant

Trdan, Stanislav

Trejo, Joann

Trenti, Annalisa

Trentini, Alessandro

Tresguerres, Jesus

Tresguerres, Jesús

Tretyakova, Natalia

Trevisan, Andrea

Triaca, Viviana

Tribulova, Narcisa

Tricarico, Domenico

Trifonov, Vladimir

Trigatti, Bernardo

Trillas, M. Isabel

Trilling, Mirko

Trimboli, Pierpaolo

Trimigno, Alessia

Trinchera, Marco

Trincone, Antonio

Trinick, John

Tripathi, Diwaker

Tripathi, Manish

Tripathi, Manoj

Trivedi, Darshan 
Trivedi, Pankaj

Trobaugh, Derek

Troiano, Giuseppe

Troisi, Jacopo

Trojanowicz, Bogusz

Trollope, Alexandra

Tronel, Claire

Troppmair, Jakob

Trubiani, Oriana

Trujillo-Rodríguez, María José

Truong, Vi Khanh

Truve, Erkki

Tryndyak, Volodymyr

Trzeciak, Anna M.

Tsafantakis, Nikolaos

Tsai, Ang-Chen

Tsai, Inn-Ho

Tsai, Kun-Ling

Tsai, Shih-chang

Tsai, Shih-Jen

Tsai, Shu-Huai

Tsai, Yuan-Chin

Tsapogas, Panagiotis

Tsatsakis, Aristides M.

Tsavkelova, Elena A.

Tse, William K.F.

Tse-Dinh, Yuk-Ching

Tseng, Te-Ming Paul

Tseng, Yi-Tang

Tsiotra, Panayoula C.

Tsirigotis, Panagiotis

Tsitsilonis, Ourania E.

Tsoulfas, George

Tsoyi, Konstantin

Tsubaki, Kazunori

Tsuboi, Masahito

Tsuchida, Sachio

Tsuchiya, Hiroyuki

Tsuchiya, Yuichi

Tsuda, Takeshi

Tsuji, Atsushi B.

Tsuji, Gaku

Tsujimoto, Hisashi

Tsukahara, Tamotsu

Tsukahara, Toshifumi

Tsutsui, Makusu

Tsutsui, Shigeyuki

Tsytsarev, Vassiliy

$\mathrm{Tu}$, Thomas

Tuanyok, Apichai

Tuccinardi, Tiziano

Tuchscherr, Lorena

Tucker, Steven

Tueros, Itziar

Tuite, Mick

Tulloh, Robert

Tunaru, Sorin

Tuncel, Aytug
Tuomikoski, Pauliina

Tuorto, Francesca

Turano, Paola

Turiel, Maurizio

Turkina, Maria V.

Turnbull, Matthew W.

Turner, Jonathan

Turner, Mark

Turoverov, Konstantin

Turowski, Patric

Turrini, Eleonora

Turroni, Silvia

Tuttolomondo, Antonino

Tuvdendorj, Demidmaa

Tuveng, Tina Rise

Tuyaerts, Sandra

Tyagi, Rahul

Tyrka, Mirosław

Tyunin, Alexey P.

Tzin, Vered

Tzinia, Athina K.

Uberti, Francesca

Uchida, Shizuka

Udumula, Venkata Reddy

Uegaki, Koichi

Ueno, Shu-ichi

Ufnal, Marcin

Uhl, Eberhard

Uhrikova, Daniela

Ulasov, Ilya

Ulf, Kahlert

Ulivieri, Cristina

Ulman, Vladimír

Ulrich, Craig

Umar, Akhilesh K

Umehara, Mikihisa

Umerska, Anita

Unchwaniwala, Nuruddin

Underwood, Mark D.

Ungefroren, Hendrik

Upadhyay, Rakesh K.

ur Rehman, Ihtesham

Urano, Daisuke

Uray, Ivan

Urban, Jill

Urban, Philippe

Urbanczyk-Lipkowska, Zofia

Urbanek, Tomasz

Urbanelli, Lorena

Urbano, Ana Margarida

Úrbez-Torres, José Ramón

Usmani, Abul

Üstün, Suayib

Usui, Soichiro

Uusitupa, Matti

Uversky, Vladimir N.

Uziel, Orit

Vaahtera, Lauri 
Václavíková, Radka

Vadivel, Kanagasabai

Vadlapudi, Aswani Dutt

Vafiadaki, Elizabeth

Vågesjö, Evelina

Vaghefi, Ehsan

Vago, Riccardo

Vaillancourt, Cathy

Vaiman, Daniel

Vainio, Seppo

Vairetti, Mariapia

Vaisar, Tomáš

Vajro, Pietro

Valabrega, Giorgio

Valdes-Lopez, Oswaldo

Vale, Filipa F.

Valentine, Rudy J.

Valentová, Kateřina

Valentovic, Monica

Valenzuela, C. Fernando

Valenzuela, Stella

Valero, Marta Sofía

Vali, Payam

Valko, Marian

Valkov, Vladimir

Valle, Adamo

Vallée, Alexandre

Vallera, Daniel

Valles, Soraya L.

Valletta, Alessio

Valverde, Ángela M.

Vamanu, Emanuel

Vamecq, Joseph

Van Aken, Olivier

Van Beuningen, Henk M.

Van Cruchten, Steven

Van Cutsem, Pierre

Van Dam, Nicole

Van De Poel, Bram

Van De Ven, Rieneke

Van Deenen, Nicole

Van Den Bergh, Hubert

Van Der Does, Anne M.

Van Der Giezen, Mark

van der Kamp, Jan Willem

Van Der Kuyl, Antoinette

Van Der Linde, Karina

Van Der Spoel, Aarnoud C.

Van Der Veer, Eric P.

Van Der Vorst, Emiel

Van Dyke, Michael W.

van Eeden, Stephan

Van Esse, Wilma

Van Eynde, Aleyde

Van Grevenynghe, Julien

Van Griensven, Martijn

Van Horn, Wade

Van Loo, Hanna M.
Van Meeteren, Laurens A.

Van Noorden, Cornelis

Van Raamsdonk, Jeremy M.

Van Rijn, Richard M.

Van Roosbroeck, Katrien

Van Royen, Niels

Van Spil, Erwin (W.E.)

Van Tine, Brian Andrew

Van Vliet, Sandra

Van Zandwijk, Nico

Van Haute, Lindsey

Vance, David E.

Vandeputte, Doris

Vandooren, Jennifer

Vaňhara, Petr

Vankayala, Raviraj

Vanková, Radomira

Vanneste, Steffen

Vanni, Roberta

Vannini, Ivan

Váradi, Györgyi

Varallyay, Eva

Varando, Gherardo

Varanini, Zeno

Varela-Ramirez, Armando

Varese, Giovanna Cristina

Varghese Chacko, Jenu

Varikuti, Sanjay

Varotsos, Costas

Varotto, Serena

Varricchio, Ettore

Varshavsky, Alexander J.

Vasaikar, Suhas

Vashist, Sandeep K.

Vasilevsky, Nicole

Vasin, Mikhail V.

Vassetzky, Yegor

Vasudevan, Ananda Ayyappan Jaguva

Vathipadiekal, Vinod

Vaudry, Hubert

Vaughan, Roger A.

Vautier, Simon

Vázquez-Marrufo, Gerardo

Vecchione, Carmine

Vecchione, Raffaele

Vechetti, Ivan

Vecoli, Cecilia

Veenman, Leo

Veenstra, Richard D.

Veeramani, Suresh

Veeraraghavan, Rengasayee

Vega Gutierrez, Sarath

Vega, Francisco M.

Vega, Gladys Mínguez

Végh, Attila Gergely

Veiga-Lopez, Almudena

Veikko, Linko

Velasco, Guillermo 
Veldkamp, Christopher

Veleeparambil, Manoj

Vélez-Cruz, Renier

Velitchkova, Maya

Velivelli, Siva

Vellante, Federica

Vemula, Harika

Venditti, Alessandro

Vennerstrom, Jonathan

Ventoso, Iván

Venuti, Aldo

Venza, Mario

Verdeguer, Francisco

Verdoucq, Lionel

Vergara, Daniele

Verhage, Leonie

Verhoeven, Adrie

Verma, Navin Kumar

Vermaas, Joshua V.

Vernetti, Lawrence A.

Veronese, Mattia

Veronesi, Fabio

Véronique, Demers-Mathieu

Véronique, Santoni

Verrey, François

Verron, Elise

Versacci, Paolo

Versino, Elisabetta

Verstegen, Monique M. A.

Verstraeten, Inge

Versura, Piera

Vervaet, Benjamin

Veskoukis, Aristidis S.

Vesper, Stephen

Vessey, Kirstan

Vetere, Amedeo

Veyron-Churlet, Romain

Vicente, Claudia

Vicente, Joao

Vickers, Mark

Victoni, Tatiana

Victor, Bruno

Vidalain, Pierre-Olivier

Vidic, Jasmina

Vieira, Maria Lúcia Carneiro

Viennois, Emilie

Viganò, Paola

Viggiano, Davide

Vignes, Michel

Vijayakumar, Sarath

Vijayavenkataraman, Sanjairaj

Villa, Chiara

Villa, Stefania

Villa-Bellosta, Ricardo

Villalobos, Carlos

Villalta, Peter

Villarino, Gonzalo

Villar-Lozano, Diego
Villar-Piqué, Anna

Villasante, Aranzazu

Villers, Agnès

Vinardell, José M.

Vinardell, Maria

Viñas, Miguel

Vincenzo, De Leo

Vinciguerra, Manlio

Vinken, Mathieu

Vinkler, Michal

Vinnedge, Lisa Privette

Virag, Laszlo

Visconti, Richard P.

Visioli, Francesco

Vistoli, Giulio

Vita, Roberto

Vitale, Alessandro

Vitale, Marilena

Vítámvás, Pavel

Vitetta, Luis

Vitry, Pauline

Vivo, Maria

Vizoso, Francisco J.

Vlachos, Ioannis

Vladimirov, Vladimir

Vliagoftis, Harissios

Vo, Minh

Voelckel, Claudia

Voelkers, Mirko

Voellger, Benjamin

Vogelaar, Christina Francisca

Voiniciuc, Cătălin

Vojta, Lea

Volarevic, Vladislav

Volk, David

Volonté, Cinzia

Von Delft, Frank

Von Eckardstein, Arnold

Von Knethen, Andreas

Von Kobbe, Cayetano

Von Zglinicki, Thomas

Vona, Barbara

Vora, Mehul

Voss, Ute

Voutsadakis, Ioannis A.

Vozza, Iole

Vrentas, Catherine E.

Vriend, Jerry

Vrontaki, Eleni

Vukojevic, Vladana

Vuts, Jozsef

Vyazovkin, Sergey

Vymetalkova, Veronika

Vyoral, Daniel

Wabiko, Hiroetsu

Wachten, Dagmar

Wada, Jun

Wada, Ken-Ichi 
Wada, Ryuichi

Waddell, Jaylyn

Wade, Rebecca

Wadehra, Madhuri

Wagh, Kshitij

Wagner, Anika

Wagner, Gabriele

Wagner, Lars

Wagner, Lars M.

Wahli, Walter

Wakao, Masahiro

Wakimoto, Hiroaki

Wako, Hiroshi

Wakshlag, Joseph

Walczak, Katarzyna

Walentowicz-Sadlecka, Malgorzata

Waligorski, Piotr

Walker, Chandler L.

Walker, Heather

Wallace, Nicholas

Waller, Deborah

Waller, Rachel

Wall-Medrano, Abraham

Walsh, Laurence J.

Walters, Giles

Walton, Gemma Emily

Wan, Edwin

Wan, Lam Yim

Wan, Shibiao

Wan, Xuehua

Wanders, Desiree

Wang, Bang-An

Wang, Bo

Wang, Chin-Kun

Wang, Fuyi

Wang, Guliang

Wang, Guozheng

Wang, Hsiuying

Wang, Huaimin

Wang, Hui-Min David

Wang, Jenghan

Wang, Jing

Wang, Jiou

Wang, Jun

Wang, Kai

Wang, Ling

Wang, Lisa L

Wang, Lizhu

Wang, Pengcheng

Wang, Peng-Hui

Wang, Piwen

Wang, Qiang

Wang, Qin

Wang, Qun

Wang, Ranran

Wang, Raymond Y.

Wang, Richard

Wang, Rui
Wang, San-Lang

Wang, Sheng

Wang, Shih-Wei

Wang, Shyiwu

Wang, Tsung-Jen

Wang, Wen-Der

Wang, Wenqi

Wang, Wentian

Wang, Xiaowan

Wang, Xinkun

Wang, Xiupeng

wang, xuewen

Wang, Yifei

Wang, Yi-Fen

Wang, Ying

Wang, Yun-Ming

Wang, Zhe

Wang, Zhihan

Ward, Douglas

Ward, Sara Jane

Wardill, Hannah R.

Warzecha, Tomasz

Warzecha, Zygmunt

Wase, Nishikant

Wasinger, Valerie C.

Wasternack, Claus

Wasylishen, Amanda

Watanabe, Masakatsu

Watanabe, Masatoshi

Watanabe, Masayuki

Watanabe, Miki

Waterhouse, Miguel

Waters, Michael

Watrelot, Aude Annie

Watson, Lauren

Watson, William

Wattanapitayakul, Suvara K.

Wayel, Jassem

Wdowiak, Kamil

Weaver, Jolanta

Weber, Daniel

Weber, David S.

Weber, Franz

Weber, Georg F.

Weber, John

Weber, Lynn

Webster, Marie R.

Wei, Changyong

Wei, Yonglong

Wei, Yufeng

Weigand, Julia E.

Weigert, Andreas

Weighardt, Heike

Weigmann, Benno

Weil, Michael M.

Weiskopf, Daniela

Weiss, Julia

Wellington, Cheryl 
Wellmann, Sven

Wells, James W.

Wells, Richard A.

Wells, Tim

Welsch, Ralf

Welsh, C. Jane

Welsh, Michael

Wemheuer, Franziska

Wen, Chiu-Min

Wen, Chunyi

Wen, Jianjun

Wende, Wolfgang

Wendell, Stacy Gelhaus

Weng, Mao-Lun

Weng, Xiaoyu

Wenisch, Sabine

Wenkel, Stephan

Wennmalm, Stefan

Werley, Christopher A.

Wesolowski, Robert

Wessells, Robert

Wessinger, Carolyn

West, Alison C.

West, Lori J.

West, Tim

Weyer, Andy

Whelan, Rebecca J.

White, Elizabeth

White, Peter

Whitfield, Phil

Whitford, Paul Charles

Wibowo, David

Wickner, Reed B.

Widmer, Hans Rudolf

Wie, Myung-Bok

Wieckiewicz, Mieszko

Wieczorek, Edyta

Wieczorek, Rafal M.

Wiemer, Erik A.C.

Wierichs, Richard

Wieser, Rotraud

Wietrzyk, Joanna

Wiggins, Greg

Wiggs, Michael

Wigle, Jeffrey

Wigley, Dale

Wijeyeskere, Sanjeeva

Wildemann, Britt

Wilker, Jonathan

Wilkinson, J. M.

Wilkinson, Mark D.

Willberg-Keyriläinen, Pia

Willert, Karl

Willette, Auriel A.

Williams, Christopher S.

Williams, Stephen

Williams, Steven E.

Willis, Rohan
Willison, Hugh

Wilson, Clive

Wilson, Eleanor M.P.

Wilson, Ted

Winchester, Laura M.

Winek, Katarzyna

Winkler, Johannes

Winship, Amy

Wińska, Katarzyna

Winuthayanon, Wipawee

Wischhusen, Joerg

Wischik, Claude

Wiśniewski, Marek

Witczak, Carol

Witeska, Małgorzata

Witowski, Jan Sylwester

Witt, Martin

Witte, Hartmut

Witten, Paul Eckhard

Wittstock, Ute

Wlaz, Piotr

Włoga, Dorota

Wodrich, Harald

Wójkowska, Dagmara Weronika

Wojtas, Bartosz

Wojtyczka, Robert

Wojtyla, Łukasz

Wölfler, Albert

Wolińska, Agnieszka

Wolley, Martin

Won, Kyu Chang

Won, Kyung Jong

Wong, Alan

Wong, Boon-Seng

Wong, Chi-Ming

Wong, Ching-On

Wong, Chung F.

Wong, Connie

Wong, Ka-Chun

Woo, Se Joon

Woo, So-Youn

Woodhead, Jeffrey L.

Woodman, Tim

Woolbright, Benjamin L.

Worch, Remigiusz

Workman, Victoria

Worrell, Roger

Woulfe, Kathleen C.

Woźniak, Łukasz

Wright, Catherine

Wrzaczek, Michael

Wrześniok, Dorota

$\mathrm{Wu}$, Erxi

$\mathrm{Wu}$, Fen

$\mathrm{Wu}$, Guangxi

$\mathrm{Wu}$, Hannah

$\mathrm{Wu}$, Kuen-phon

$\mathrm{Wu}$, Shu 
$\mathrm{Wu}, \mathrm{Si}$

$\mathrm{Wu}$, Ting-Feng

$\mathrm{Wu}$, Tzong-Yuan

$\mathrm{Wu}, \mathrm{Yan}$

$\mathrm{Wu}$, Yanyuan

$\mathrm{Wu}, \mathrm{Yi}$-Ying

Wu, Yuh-Lin

Wunderlich, Frank

Würth, Roberto

Wybouw, Nicky

Wynes, Murry W.

Wyns, Christine

Wyrobek, Andrew J.

Wystrychowski, Grzegorz

Xavier, Nuno Manuel

Xia, Tian

Xiang, Yu

Xiao, Li

Xiao, $\mathrm{Yu}$

Xiaoli, Alus

Xie, Hong

Xie, Ran

Xie, Wankun

Xie, Zhong-Ru

Xin, Dongyue

Xin, Ran

Xing, Gengmei

Xing, Junji

Xiong, Ye

Xiong, Zhaohui

$\mathrm{Xu}, \mathrm{Bo}$

$\mathrm{Xu}$, Feng

$\mathrm{Xu}$, Huaxi

$\mathrm{Xu}$, Jing

$\mathrm{Xu}$, Jinsong

$\mathrm{Xu}$, Jintao

$\mathrm{Xu}, \mathrm{Lu}$

$\mathrm{Xu}$, Peng

$X u$, Pengfei

$\mathrm{Xu}$, Shengsheng

$\mathrm{Xu}$, Suowen

Xu, Wayne

$\mathrm{Xu}$, Wenyan

$\mathrm{Xu}, \mathrm{Xin}$

$\mathrm{Xu}, \mathrm{Yi}$

$\mathrm{Xu}$, Yungang

Xu, Zhijie

Xu, Zhiping

Xuan, Tran Dang

Yabe, Kimiko

Yadav, Vikramaditya G.

Yadgarov, Lena

Yaegashi, Hajime

Yager, Dorne

Yagi, Akira

Yago, Toru

Yagüe, Ernesto

Yaish, Mahmoud W.
Yakovlev, Igor A.

Yalowich, Jack C.

Yamaguchi, Junpei

Yamaji, Toshiyuki

Yamakuchi, Munekazu

Yamamoto, Suguru

Yamamoto, Yusuke

Yamamura, Aya

Yamamura, Hisao

Yamamura, Shohei

Yamamura, Soichiro

Yamanouchi, Keitaro

Yamasaki, Masahiro

Yamashiro, Sawako

Yamashiro, Takashi

Yamasu, Kyo

Yamauchi, Takahiro

Yamawaki, Hideyuki

Yamazaki, Yu

Yan, Dayun

Yan, Dongqing

Yan, Shengmin

Yanda, Murali

Yang, Chih-Tsung

Yang, Chin-Ying

Yang, Deng-Ho

Yang, Gabsik

Yang, Guang

Yang, Hui Lin

Yang, Jae Wook

Yang, Jenq-Lin

Yang, Jen-Tsung

Yang, Jung-Wu

Yang, Jun-Yi

Yang, Jye-Shane

Yang, Kun

Yang, Lee-Wei

Yang, Lijiang

Yang, Qiyuan

Yang, Rong-Sen

Yang, Seung Hwan

Yang, Sherry $X$.

Yang, Shuyi

Yang, Sze-Ming Mildred

Yang, Ta-I

Yang, Tao

Yang, Tuo

Yang, Wancai

Yang, Wei-hsiung

Yang, Xia

Yang, Ye

Yang, Yongkang

Yang, Yueh-Hsun Kevin

Yang, Zhihong

Yann, Audic

Yannakopoulou, Konstantina

Yanni, Souzan

Yano, Tohru 
Yao, Hiroshi

Yao, Jiayi

Yao, Masahiro

Yao, Peng

Yao, Yanhua

Yao, Zhili

Yarowsky, Paul

Yasuda, Kazuki

Yasuda, Takako

Yasuhara, Takao

Yates, Clayton

Yazlovitskaya, Eugenia M.

Ye, Adam

Ye, Haobin

Ye, Yanqi

Yeckel, Catherine Weikart

Yeh, Chih-ko

Yeh, Elizabeth S.

Yeh, Hsin-Hung

Yeh, Shu-Lan

Yeh, Yin-Ting

Yehuda, Shoenfeld

Yeligar, Samantha

Yen, Feng-Lin

Yen, Meng-Chi

Yeo, Syn

Yew, P. Renee

Yi, Ae-Kyung

Yi, Haowei

Yi, Young-Su

Yilmaz, Bahtiyar

Yin, Chengqian

Ying, Tiejin

Yokohira, Masanao

Yokoi, Shuji

Yokoi, Tsuyoshi

Yokoo, Hiroki

Yokota, Shinso

Yonekura, Shinichi

Yoneshiro, Takeshi

Yong, Jeongsik

Yoo, Ki-Oug

Yoo, Soonmoon

Yoo, Tag Keun

Yool, Andrea

Yoon, Do-Young

Yoon, Hyonok

Yoon, In-Soo

Yoon, Je-Hyun

Yoon, Tae Ki

Yoshida, Hiderou

Yoshida, Katsunori

Yoshida, Manabu

Yoshida, Takahiro

Yoshida, Toshinori

Yoshida, Yamato

Yoshihara, Toshinori

Yoshino, Hironori
Yoshioka, Ken-ichi

Yoshitomi, Hiroyuki

Yosten, Gina

You, Hyunju

You, Sylvaine

You, Xiaona

Young, Arabella

Young, Bryan

Young, Bryan G.

Young, Jason C.

Young, Kaylene

Yu, Bin

Yu, Chia-Li

Yu, Feiqiao Brian

$\mathrm{Yu}$, Gang

Yu, Jian

$\mathrm{Yu}$, Richard

$\mathrm{Yu}$, Seung-Young

$\mathrm{Yu}$, Victor

Yu, Yan Ping

Yu, Yang

Yu, Yuan-Hsiang

Yuan, Bo

Yuan, Hanna S.

Yuan, Yaxia

Yuan, Zhihong

Yuba, Eiji

Yue, Patrick

Yuen, John Wm

Yuen, Karen Wing Yee

Yumoto, Hiromichi

Yun, Chawon

Yun, Hongseok

Yun, Hyungdon

Yurchenco, Peter D.

Yushenova, Irina

Yuspa, Stuart H.

Zabetakis, Ioannis

Zaccai, Michele

Zaccheroni, Nelsi

Zacchia, Miriam

Zacconi, Flavia C.

Zachou, Kalliopi

Zafar, Muhammad

Zagidullin, Naufal

Zahedi, Kamyar

Zaibi, Mohamed Sghaier

Zaid, Hilal

Zaidi, Syed Shan-e-Ali

Zakhari, Samir

Zaki, Hasan

Žáková, Lenka

Zakrocka, Izabela

Zakrzewicz, Dariusz

Zallot, Remi G.

Zambonelli, Paolo

Zambrano, Samuel

Żamojć, Krzysztof 
Zamyatnin, Jr., Andrey A.

Zanelli, Cleslei Fernando

Zang, Liqing

Zani, Bianca Maria

Zaniboni, Massimiliano

Zanin, Laura

Zannetti, Antonella

Zapata, Carlos

Zaphiropoulos, Peter

Zapico, Sara C.

Zarogoulidis, Paul

Zarra, Ignacio

Zasloff, Michael

Zaucke, Frank

Zauderer, Marjorie G.

Zavattaro, Elisa

Zaza, Gianluigi

Zdarta, Jakub

Zebisch, Armin

Zehbe, Ingeborg

Zeidán-Chuliá, Fares

Zeier, Jürgen

Zeilinger, Carsten

Zemel, Michael

Zenclussen, Ana

Zeng, Hongmei

Zeng, Hulie

Zeppieri, Marco

Zeugolis, Dimitrios

Zgaga, Lina

Zhang, Bo

Zhang, Caiguo

Zhang, Chi

Zhang, Deqiang

Zhang, Dianbao

Zhang, Duo

Zhang, Hengyou

Zhang, Huanmin

Zhang, JianMin

Zhang, Jian-min

Zhang, Jinnan

Zhang, Junran

Zhang, Ke

Zhang, Kezhong

Zhang, Lei

Zhang, Ruiyong

Zhang, Shuling

Zhang, Sui

Zhang, Wei

Zhang, Weijie

Zhang, Wencai

Zhang, Xiang

Zhang, Xiaohui

Zhang, Xiaoyan

Zhang, Xuming

Zhang, Yali

Zhang, Ye

Zhang, Yingyue
Zhang, Yixiang

Zhang, Yixuan

Zhang, Yuan-Ming

Zhang, Yunkai

Zhang, Zhiyong

Zhang, Zhiyue

Zhao, Baoyin

Zhao, Jichao

Zhao, Liang

Zhao, Min

Zhao, Ningning

Zhao, Qi

Zhao, Qingnan

Zhao, Ruiming

Zhao, Shan

Zhao, Sifeng

Zhao, Wenxiu

Zhao, Zongmin

Zheng, Guoping

Zheng, Huanquan

Zheng, Shilong

Zheng, Yue

Zhivkova, Zvetanka D.

Zhong, Haizhen

Zhong, Jinshun

Zhong, Tuhua

Zhong, Wei-Zhu

Zhou, Bangjun

Zhou, Beiyan

Zhou, Dezhong

Zhou, Guangming

Zhou, Heshan Sam

Zhou, Jia

Zhou, LinLi

Zhou, Shuanhu

Zhou, Wenchao

Zhou, Xue-Rong

Zhou, Yi-hong

Zhou, Yubin

Zhou, Zhichao

Zhou, Zhixiong

Zhu, Bing

Zhu, Guo-Zhang

Zhu, Hongyan

Zhu, Jingen

Zhu, Qiuyu

Zhu, Wei

Zhu, Xiangwei

Zhu, Xiaoping

Žiarovská, Jana

Ziche, Marina

Zichel, Ran

Ziebell, Heiko

Ziegler, Patrick

Zielenkiewicz, Piotr

Zielińska-Pisklak, Monika A.

Zima, Aneta

Zimmer, Gert 
Zimmer, Marc

Zimmer, Warren E.

Zimmerberg, Joshua

Zimmermann, Herbert

Zloh, Mire

Zmijewski, Michal

Zollner, Thomas

Zoltan-Istvan, Szabo

Zorc, Branka

Zordoky, Beshay

Zou, Quan

Zsolnai, Attila

Zubieta, Chloe
Zugaza, J. L.

Žukauskaitè, Asta

Zulli, A.

Züllig, Richard

Zúñiga, Sonia

Zuo, Jian

Zupko, Istvan

Zuppinger, Christian

Zuvela, Petar

Zwack, Paul

Zweier, Christiane

Żyżelewicz, Dorota

(C) 2019 by the authors. Licensee MDPI, Basel, Switzerland. This article is an open access article distributed under the terms and conditions of the Creative Commons Attribution (CC BY) license (http://creativecommons.org/licenses/by/4.0/). 